\title{
The 4-Parameter Ionospheric Channel Model: Part 1- Theory and Simulation
}

You cannot significantly improve modeling unless you can significantly improve the methodology of modeling. - Ilir Progri

\author{
Ilir F. Progri \\ Giftet Inc., 5 Euclid Ave. \#3, Worcester, MA 01610, USA \\ ORCID: 0000-0001-5197-1278
}

Correspondence should be addressed to Ilir Progri; iprogri@giftet.com

Received December 23, 2017; Presented Feb. 1, 2018; Revised January 12, 2018-May 7, 2019, Accepted July 16, 2019;

Published November 1, 2019.

Scientific Editor-in-Chief/Editor: Ilir F. Progri

Copyright (C) 2019 Giftet Inc. All rights reserved. This work may not be translated or copied in whole or in part without written permission to the publisher (Giftet Inc., 5 Euclid Ave. \#3, Worcester, MA 01610, USA), except for brief excerpts in connection with reviews or scholarly analysis. Use in connection with any form of information storage and retrieval, electronic adaptation, computer software or by similar or dissimilar methodology now known or hereafter developed is forbidden. The use of the publication of trade names, trademarks, service marks, or similar terms, even if they are not identified as such, is not to be taken as an expression of opinion as to whether or not they are subject to proprietary rights.

The 1-parameter ionospheric channel model that is based on estimating the ionospheric delay is insufficient to enable the GNSS receiver to reliably acquire and track GNSS signals under severe ionospheric conditions.

In order to solve this problem Giftet Inc. is proposing the 4-parameter ionospheric model. The 4-parameter ionospheric model is based on estimating four parameters: ionospheric delay, Doppler, amplitude, and phase scintillation. The 4-parameter ionospheric model ${ }^{i}$ presents a revolutionary advancement of our understanding of the ionospheric effects in the RF signals. In order to accurately estimate the ionospheric Doppler we have performed some of the most amazing mathematical calculations.

Afterwards, the theory and simulation, presented in this paper, are completely verified and accurately tested, the implementation thereof in a single frequency GNSS receiver will enable it to perform just as good as or even better than a differential GNSS receiver.

Index Terms - 4-parameter, ionospheric, channel, model, delay, Doppler, amplitude, phase, scintillation, MATLAB.

\section{Introduction}

Ionosphere : is the part of the atmosphere that: (1) stretches from 50 to $1,000 \mathrm{~km}$ (31.06855 to $621.371 \mathrm{mi} ; 164,042$ to $3,280,840 \mathrm{ft}$ ), (2) is ionized (i.e.; contains ions and electrons) by solar radiation, typically overlaps both the exosphere and the thermosphere; (3) forms the inner edge of the magnetosphere; (4) greatly influences, for example, radio propagation on the Earth; and (5) is responsible for auroras (see Figs. 1 and 2) [1], [2].

The radio window consists of frequencies which range from 


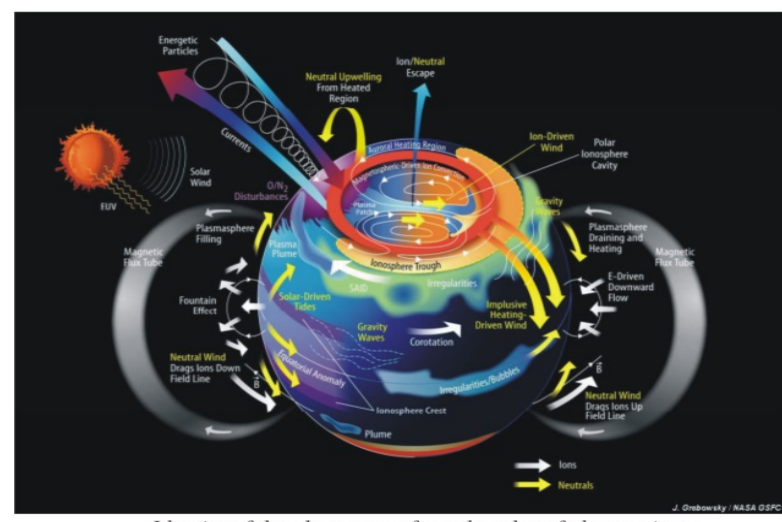

Identity of the phenomena from decades of observation.

Fig. 1. Identity of the plasma and ionospheric phenomena from decades of observations. Courtesy of NASA 2009.

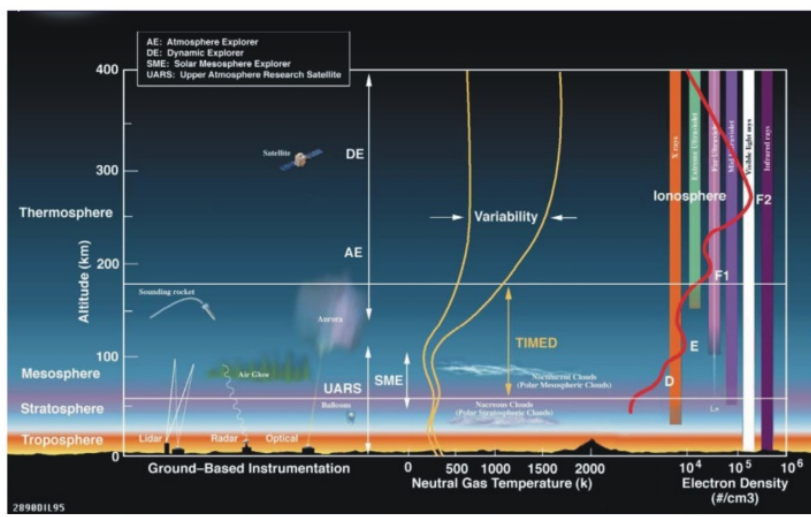

Ground based instrumentation; natural gas temperature $(K)$

Electron density vs. Altitude (km). AE: Atmosphere Explore

DE: Dynamic Explorer, SME: Solar Mesosphere Explorere, UARS: Upper Atmosphere Research Satellite. Courtesy of NASA 2009.

Fig. 2. Ground based instrumentation; natural gas temperature (K); Electron density vs. Altitude (km). AE: Atmosphere Explorer DE: Dynamic Explorer; SME: Solar Mesosphere Explorer; UARS: Upper Atmosphere Research Satellite. Courtesy of NASA 2009.

about $5 \mathrm{MHz}(5$ million $\mathrm{Hz}$ ) to $30 \mathrm{GHz}$ (30 billion $\mathrm{Hz}$ ). The low-frequency end of the window is limited by signals being reflected by the ionosphere [2] back into space or Earth, while the upper limit is caused by absorption of the radio waves by water vapor [3] and carbon dioxide in the atmosphere. As atmospheric conditions change the radio window can expand or shrink. On clear days with perfect conditions signals as high as $300 \mathrm{GHz}$ have been detected (see Figs. 1-3) [1].

Normally radio "ground waves" propagate along the surface as creeping waves; i.e., they are only diffracted around the curvature of the earth, which is the reason why early long distance radio communications used long wavelengths with the best known exception of the HF (3-30 MHz) waves being reflected by the ionosphere [2], [4].

The reduced refractive index [5] due to lower densities at the

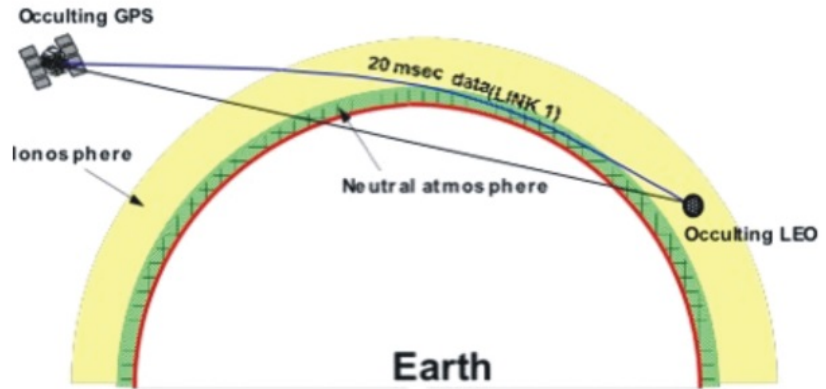

Basic measurement principle: Deduce atmospheric properties based on precise measurement of phase delay and amplitude. Courtesy of NOAA 2007

Fig. 3. Basis ionospheric measurement principle: deduce atmospheric properties based on precise measurement of phase delay and amplitude. Courtesy of NOAA 2007.
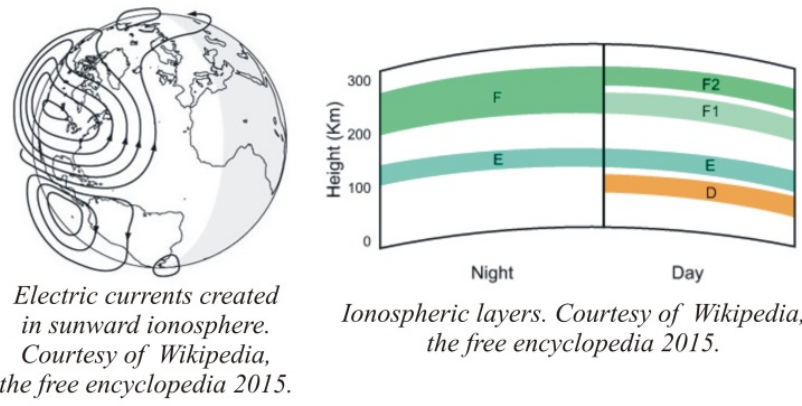

Ionospheric layers. Courtesy of Wikipedia the free encyclopedia 2015.

Fig. 4. Electric currents created in sunward ionosphere (left). Ionospheric layers vs height (or altitude) $(\mathrm{km})$ (right). Courtesy of Wikipedia the free encyclopedia 2015.

higher altitudes in the Earth's atmosphere bends the signals back toward the Earth. When signals encounter a higher refractive index [5] layer duct, tend to remain in that layer because of the reflection [6] and refraction [6] encountered at the boundary with a lower refractive index material and vice versa. In some weather conditions, such as inversion layers, density changes so rapidly that waves are guided around the curvature of the earth at a constant [8] altitude [4].

Ionospheric reflection : is a bending, through a complex process involving reflection and refraction [6], of electromagnetic waves propagating in the ionosphere [2] back toward the Earth [6]. The amount of bending depends on the extent of the penetration (which is a function of frequency), the angle of incidence, the polarization of the wave, and ionospheric conditions, such as the ionization density. It is negatively affected by incidents of ionospheric absorption [6].

Ionospheric absorption/dispersion (or ISAB/ISD) is the scientific name for absorption/ dispersion occurring as a result of the interaction between various types of electromagnetic waves and the free electrons in the ionosphere [2], which can 
interfere with radio transmissions which results in a significant loss/dispersion of the signal power [9].

Ionospheric absorption/dispersion is of critical importance when radio networks, telecommunication systems or interlinked radio systems are being planned, particularly when trying to determine propagation conditions [9].

The ionosphere [2] can be described as an area of the atmosphere in which radio waves on shortwave bands are refracted or reflected back to earth (see Figs. 1-4). As a result of this reflection, which is often the key in the long-distance propagation of radio waves, some of the shortwave signal strength is decreased. In this regard, ISAB/ISD is the primary limiting factor in radio propagation [9].

ISAB/ISD is only a factor in the period of the day where radio signals travel through the portion of the ionosphere [2] facing the sun. The solar wind and radiation cause the ionosphere [2] to become charged with electrons in the first place. At night, the atmosphere becomes drained of its charge, and radio signals can go much further with less loss of signal. In particular, low wavelength signals that would be attenuated to nothing during the day will be received much further away at night [9]. The specific amount of attenuation [10] can be derived as a function of the inverse-square law. The lower the frequency is, the greater the attenuation becomes [9], [10].

Ionospheric errors are caused by delays or changes in the RF signals' time of arrival, Doppler shift, amplitude, and phase traversing the ionosphere [2], whose electron content is a function of the amount of incident solar radiation. Therefore, the ionospheric delay [11]-[13], Doppler shift, amplitude, and phase change with latitude, longitude, time of day, season (or day) of the year and, year of the millennium, following the 11.1 years solar cycle, with higher values by day (at around $14 \mathrm{~h} 00 \mathrm{~m}$ local time), during the summer and at the peaks of the solar cycle. From these three periodic changes, the dominant one is diurnal variability, following the variation in incident solar radiation [13], [14].

Superimposed on these periodic changes, severe magnetic storms occur a few times (generally not more than four times) during each 11.1 years solar cycle, causing extreme delays on GPS signals, in addition to amplitude fading and scintillation. These magnetic storms affect mainly the auroral latitudes (around the geomagnetic poles) and "for some unknown reason (...) occur more frequently during the declining phase of the solar cycle" (see Figs. 1-4) [13], [14].

Ionospheric propagation vertical delays are typically 20-30 $m$ during the day and 3-6 $m$ at night [16]. These errors are entirely compensated by DGPS and their lack of correlation with time, for periods of tens of minutes, is very low, because the ionospheric delay [11] does not change significantly on such timescales - except in the case of major magnetic storms during which the TEC changes rapidly. Dusk [17] and dawn [18] are the periods when the temporal lack of correlation of the ionospheric error is generally higher, because the ionosphere [2] re-configures itself, but even then the decorrelation is not significant. Although the de-correlation of the ionospheric errors with time has a much smaller effect than its de-correlation with distance, this is the error which contributes the most to the growth of DGPS errors with age of corrections [13], [14].

Naturally, one might ask, why does not a single frequency GNSS receiver perform just as good as a DGPS receiver? I.e., why is not a single frequency GNSS receiver capable of performing the same corrections as a DGPS receiver? The answer lies in this fact that the channel model of a single frequency GNSS receiver is currently based on a 1-parameter ionospheric channel model. The 1-parameter ionospheric channel model that is based on estimating the ionospheric delay is insufficient to enable the GNSS receiver to reliably acquire and track GNSS signals under severe ionospheric conditions.

In order to solve this problem Giftet Inc. is proposing the 4parameter ionospheric model. The 4-parameter ionospheric model is based on estimating four parameters: ionospheric delay, Doppler, amplitude, and phase scintillation. The 4parameter ionospheric model presents a revolutionary advancement of our understanding of the ionospheric effects in the RF signals. In order to accurately estimate the ionospheric Doppler, we have performed some of the most amazing mathematical calculations.

This paper presents one of the most daunting tasks ever undertaken by a single scholar to very accurately model, analyze, and simulate the entire description of the ionospheric channel effects.

Afterwards the theory and simulation, presented in this paper, are completely verified and accurately tested, the implementation thereof in a single frequency GNSS receiver will enable it to perform just as good as or even better than a differential GNSS receiver (Progri 2018, [19]). 


\section{Description of RF Geolocation Propagation Channel Model}

When an RF signal propagates from an RF transmitter (typically an RF (or GNSS) satellite orbiting the Earth at approximately 20,200 $\mathrm{km}$ altitude from the surface of the Earth) to a RF receiver antenna (typically most receivers are located from 0 to $30 \mathrm{~km}$ from the surface of the Earth), it suffers from degradation effects, such as, free space path loss, absorption and refraction from the atmosphere, reflection and masking from surrounding objects such as trees and buildings, jamming, and environmental noise as shown in Figs. 5-8.

\subsection{Description of an ideal RF geolocation transmitted signal}

Initially, we start with the description of the ideal RF geolocation propagation channel model.

The analytical expression of the ideal transmitted RF signal (or IS) can be written as the product of two components: (1) ideal baseband signal (or IBS), $\underbrace{s_{1}=S_{0} e^{j \phi_{0}}}_{\text {IBS }}$, and (2) ideal carrier signal (or ICS), $\underbrace{s_{2}\left(t ; 1,0, \omega_{c}\right)=1 \times e^{j\left(0+\omega_{c} t\right)}}_{\text {ICS }}$,

$$
\begin{aligned}
s(t) & =S_{0} e^{j\left(\phi_{0}+\omega_{c} t\right)} \\
& =\underbrace{S_{0} e^{j \phi_{0}}}_{\text {IBS }} \underbrace{1 \times e^{j\left(0+\omega_{c} t\right)}}_{\text {ICS }} \\
& =\underbrace{s_{1}}_{\text {IBS }} \underbrace{S_{2}\left(t ; 1,0, \omega_{c}\right)}_{\text {ICS }}
\end{aligned}
$$

The unknowns in (1) are as follows: (1) $t$ is the time in [s]; (2) $S_{0}$ is amplitude in [V]; (3) $\phi_{0}$ is phase in [rad]; (4) $\omega_{c}$ is the radiant carrier frequency in $[\mathrm{rad} / \mathrm{s}]$ of the transmitted GPS (or GNSS) signal respectively.

The first main assumption is as follows: IBS is a timeinvariant quantity; i.e., it only contains the desired signal amplitude, $S_{0}$, and (initial) phase, $\phi_{0}$; ICS contains the desired signal carrier [radiant] frequency, $\omega_{c}$, and time (or time stamp), $t$.

The second main assumption is that, in the description of IBS itself the desired signal amplitude, $S_{0}$, and phase, $\phi_{0}$, are also independent; in the description of ICS the desired signal carrier [radiant] frequency, $\omega_{c}$, and time stamp, $t$, are also independent; however, their product produces the accumulated carrier phase, $\phi$; and since, $\omega_{c}$ is constant the product (or correlated) information in the accumulated carrier phase, $\phi$, gives precise information about the time stamp, $t$.

Third, in an ideal environment the ideal RF geolocation propagation channel model can be completely characterized by means of four main parameters: (1) desired signal amplitude, $S_{0},(2)$ initial phase, $\phi_{0},(3)$ carrier [radiant] frequency, $\omega_{c}$, and (4) time stamp, $t$.

Fourth, in an ideal (i.e., linear and homogenous) environment it is straightforward to separate these four independent parameters; namely, the signal amplitude, $S_{0}$, initial phase, $\phi_{0}$, frequency, $\omega_{c}$, and time stamp (or just time), $t$; the first and the most important attribute of modeling the received $R F$ signal is to come up with a novel, original, methodical, and useful method of separating, these four independent parameters, $S_{0}, \phi_{0}, \omega_{c}$, and $t$, from other nonlinear and heterogeneous parameters of the environment; hence, the description of the a realistic RF geolocation propagation channel model.

\subsection{Description of free-space path loss and ideal propagation channel model}

Before we consider the realistic propagation, channel let us first present the description of free-space path loss channel model.

The free space path loss, $Q_{0}$, [20] (or the lossless environment parameter) provides the relation due to inverse square law [21] as given by

$$
\begin{aligned}
Q_{0} & =\frac{P_{r}}{P_{t}} \\
& =\frac{S}{S 4 \pi R^{2}} \frac{\lambda^{2}}{4 \pi} \\
& =\left(\frac{\lambda}{4 \pi R}\right)^{2} \\
& =\left(\frac{c}{2 R \omega}\right)^{2} \\
& =\left(\frac{c}{4 \pi R f}\right)^{2}
\end{aligned}
$$

where (1) $P_{t}$ is the transmitted signal power in [W] [22]; (2) $P_{r}$ is the received signal power in [W]; (3) $S$ is the spreading of the electromagnetic energy in $\left[\mathrm{W} / \mathrm{m}^{2}\right]$; (4) $\lambda$ is the wavelength $[\mathrm{m}]$; (5) $R$ is the transmitter receiver distance (or the line-of-sight (LOS) vector magnitude) in [m]; (6) $c$ is the speed of light (SOL) in vacuum $(299,792,458 \mathrm{~m} / \mathrm{s}$ [22]); (7) $\omega$ is the radiant frequency $\omega=2 \pi f$ in $[\mathrm{rad} / \mathrm{s}]$; and (8) $f$ is the frequency in $[\mathrm{Hz}]$.

In an ideal free space environment, the free space path loss, $Q_{0}$, is the only quantity that completely characterizes or describes the physics of the propagation of the channel model; 


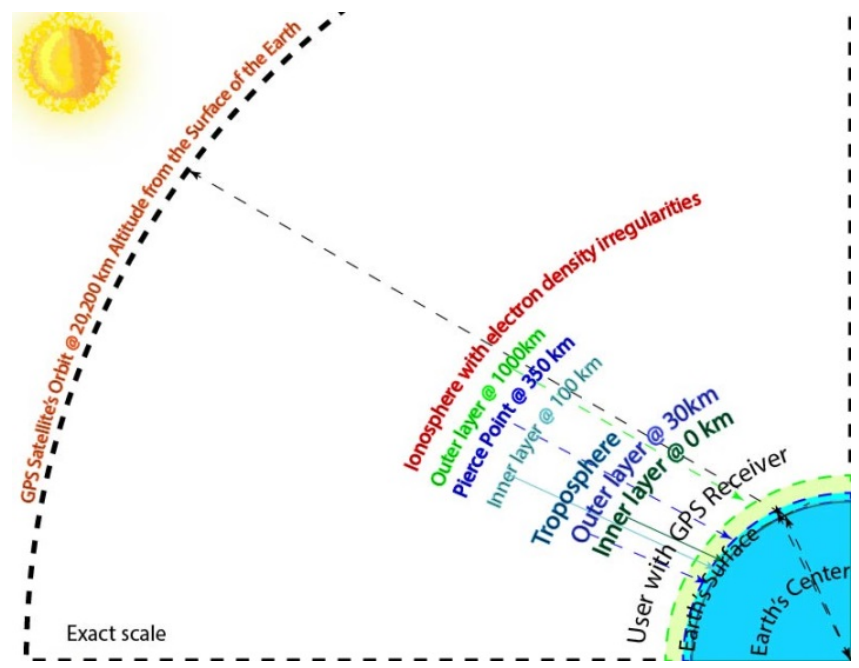

Fig. 5. RF L-Band (1-2 GHz) ionospheric propagation channel model 1.

(Exact scale).

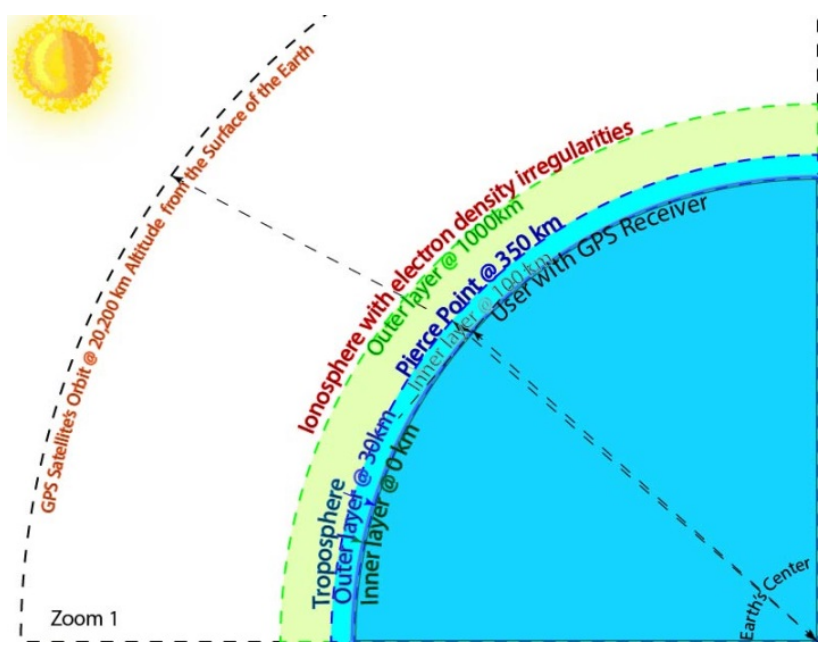

Fig. 6. RF L-Band (1-2 GHz) ionospheric propagation channel model 1.

(Zoom 1).

i.e., an ideal free space environment is in fact a one channel parameter.

\subsection{Description of an ideal $R F$ received signal}

Before we consider the realistic received signal let us present the description of an ideal RF received signal (or IRS).

First, IRS or $u_{0}\left(t ; A, \varphi, \tau, \omega_{c}\right)$ represents the received signal in vacuum; i.e., as if there were no ionosphere, or troposphere, or multipath or anything else; i.e., including only the inverse square-law propagation [21] in vacuum, which can be expressed as a product of the ideal received BS (or IRBS), $u_{1}$, which is only a function of $\left(A_{1}, \varphi_{1}\right)$ and ideal received CS (or IRCS $), u_{2}(t)$, which is a function of $\left(A_{2}, \varphi_{2}, \tau, \omega_{c}\right)$

$$
\begin{aligned}
u_{0}(t) & =\underbrace{u_{1}}_{\operatorname{IRBS}} \underbrace{u_{2}(t)}_{\operatorname{IRCS}} \\
& =A\left(t ; \tau, \omega_{c}\right) e^{j \varphi\left(t ; \tau, \omega_{c}\right)}
\end{aligned}
$$

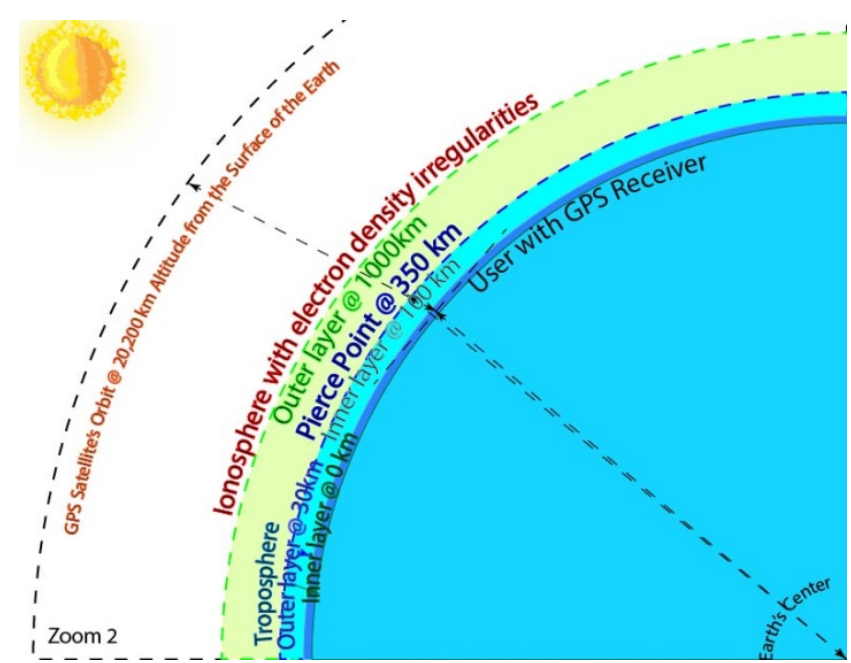

Fig. 7. RF L-Band (1-2 GHz) ionospheric propagation channel model 1.

(Zoom 2).

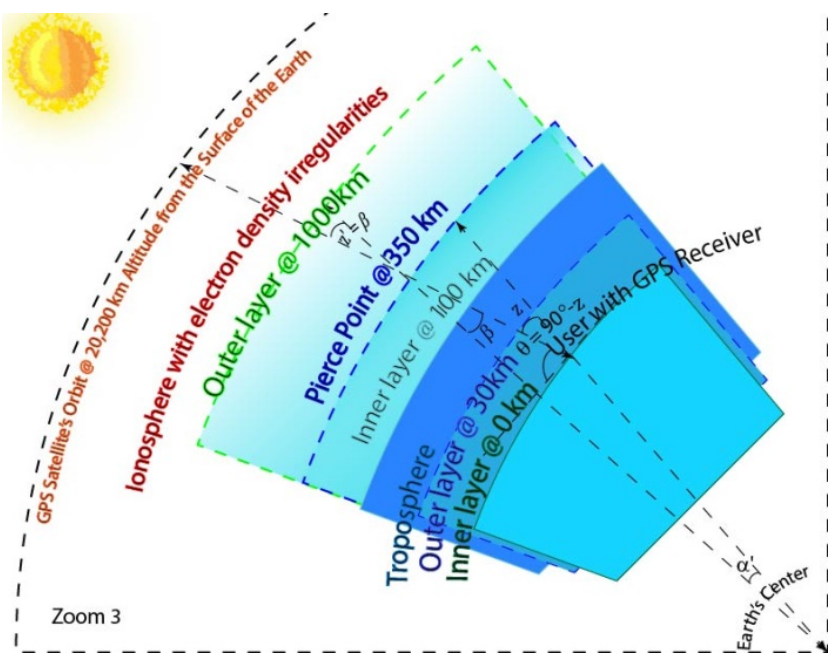

Fig. 8. RF L-Band (1-2 GHz) ionospheric propagation channel model 1. (Zoom 3).

$$
\begin{aligned}
& =A\left(t ; \tau, \omega_{c}\right) \mathrm{e}^{j \underbrace{\left[\phi_{0}+\omega_{c}\left(t-\frac{R}{c}\right)\right]}_{\varphi\left(t ; \omega_{c}, \tau\right)}} \\
& =\frac{A\left(t ; \tau, \omega_{c}\right)}{S_{0}} s\left(t-\tau ; S_{0}, \phi_{0}, \omega_{c}\right) \\
& =\frac{A\left(t ; \tau, \omega_{c}\right)}{S_{0}} S_{0} \mathrm{e}^{j\left[\phi_{0}+\omega_{c}(t-\tau)\right]} \\
& =\frac{A\left(t ; \tau, \omega_{c}\right)}{S_{0}} \underbrace{S_{0} \mathrm{e}^{j \phi_{0}}}_{\text {IBS }} \underbrace{\mathrm{e}^{j\left[\omega_{c}(t-\tau)\right]}}_{\text {ICS }} \\
& =\frac{A\left(t ; \tau, \omega_{c}\right)}{S_{0}} \underbrace{S_{0} \mathrm{e}^{j \phi_{0}}}_{\text {IBS }} \underbrace{S_{2}\left(t-\tau ; 1,0, \omega_{c}\right)}_{\text {ICS }} \\
& =A\left(t ; \tau, \omega_{c}\right) \underbrace{\frac{S_{0} \mathrm{e}^{j \phi_{0}}}{S_{0}}}_{\text {IBS }} \underbrace{S_{2}\left(t-\tau ; 1,0, \omega_{c}\right)}_{\text {ICS }} \\
& =\underbrace{A_{0} \mathrm{e}^{j \phi_{0}}}_{\text {IBS }} \underbrace{\delta A_{0}\left(t ; \tau, \omega_{c}\right) s_{2}\left(t-\tau ; 1,0, \omega_{c}\right)}_{\text {ICS }}
\end{aligned}
$$


where $A\left(t ; \tau, \omega_{c}\right)$ can be computed as the square root of the transmitter-receiver antenna gain, $G_{t r}$, and free space path loss, $Q_{0}$, given by (2)

$$
\begin{aligned}
A\left(t ; \tau, \omega_{c}\right) & =\sqrt{G_{t r} Q_{0}} \\
& =\sqrt{G_{t r}} \frac{c}{2 R \omega_{c}} \\
& =\frac{\sqrt{G_{t r}}}{2 \omega_{c} \tau(t)} \\
& =\underbrace{\left[A_{0} \equiv \frac{\sqrt{G_{t r}}}{2}\right]}_{\mathrm{IBS}} \underbrace{\left[\delta A_{0}(t) \equiv \frac{1}{\omega_{c} \tau}\right]}_{\mathrm{ICS}}
\end{aligned}
$$

where $G_{t r}$ is the transmitting and receiving antenna gain given by

$G_{t r}=G_{t} G_{r}$

where $G_{t}$ is the transmitting antenna gain in $[\mathrm{dB}]$ (or $[\mathrm{dBm}]$ ), $G_{r}$ is the receiving antenna gain in $[\mathrm{dB}]$ (or $[\mathrm{dBm}]$ ).

Hence, the IRS or $u_{0}(t)$ is given by

$$
\begin{aligned}
u_{0}(t) & =\underbrace{\frac{\sqrt{G_{t r}}}{2} \frac{s_{1}\left(S_{0}, \phi_{0}\right)}{S_{0}}}_{\text {IRBS }} \underbrace{\frac{s_{2}\left(t-\tau, \omega_{c}\right)}{\omega_{c} \tau(t)}}_{\text {IRCS }} \\
& =\underbrace{\frac{\sqrt{G_{t r}}}{2} \frac{S_{0} \mathrm{e}^{j \phi_{0}}}{S_{0}}}_{\text {IRBS }} \underbrace{\frac{e^{j\left[\omega_{c}(t-\tau)\right]}}{\omega_{c} \tau(t)}}_{\text {IRCS }} \\
& =\underbrace{A_{1} e^{j \varphi_{1}}}_{\text {IRBS }} \underbrace{\frac{\mathrm{e}^{j\left[\omega_{c}(t-\tau)\right]}}{\omega_{c} \tau}}_{\text {IRCS }}
\end{aligned}
$$

Equating both sides of (3) and (6) we have the final expression of the IRBS, $u_{1}$, and IRCS, $u_{2}(t)$, as follows

$$
u_{1}=A_{1} \mathrm{e}^{j \phi_{1}}
$$

And

$u_{2}(t)=A_{2}\left(t ; \tau, \omega_{c}\right) \mathrm{e}^{j \varphi_{2}\left(t ; \tau, \omega_{c}\right)}$

$$
=\frac{1}{\omega_{c} \tau} \mathrm{e}^{j\left[\omega_{c}(t-\tau)\right]}
$$

We can notice that the primarily decrease in signal amplitude comes from the LOS signal phase, $\phi_{c}=\omega_{c} \tau$, in [rad] or

$A_{2}\left(t ; \tau, \omega_{c}\right)=\frac{1}{\omega_{c} \tau(t)}$

and

$\varphi_{2}\left(t ; \tau, \omega_{c}\right)=\omega_{c}(t-\tau)$

Finally, equating both sides of (8), (8)-(10) we finally obtain

$A_{1}=A_{0}=\frac{\sqrt{G_{t r}}}{2}$

And

$$
A_{2}\left(t ; \tau, \omega_{c}\right)=\delta A_{0}\left(t ; \tau, \omega_{c}\right)=\left(\phi_{c}=\omega_{c} \tau\right)^{-1}
$$

In an ideal RF propagation channel, we should be able to precisely know (or estimate) four "independent" ii signal parameters $A(t), \varphi(t), \tau(t)$, and $\omega_{c}$ as follows:

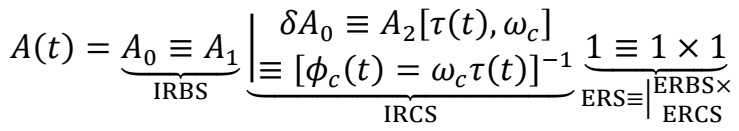

$$
\begin{aligned}
& \varphi(t)=\underbrace{\varphi_{0} \equiv \varphi_{1}}_{\text {IRBS }}+\underbrace{\delta \varphi_{0}=\varphi_{2}\left[\tau(t), \omega_{c}\right]}_{\text {IRCS }}+\underbrace{0 \equiv 0+0}_{\text {ERS } \equiv \mid \begin{array}{c}
\text { ERBS } \\
\text { ERCS }
\end{array}} \\
& =\underbrace{\phi_{0}}_{\text {IRBS }}+\underbrace{\omega_{c}[t-\tau(t)]}_{\text {IRCS }}
\end{aligned}
$$

$\tau(t)=\underbrace{0}_{\text {IBS }}+\underbrace{\frac{R(t)}{c}}_{\text {ICS }}+\underbrace{0}_{\text {ERBS }}+\underbrace{0}_{\text {ERCS }}$

$\omega_{c}=\underbrace{0}_{\text {IBS }}+\underbrace{2 \pi f_{c}}_{\text {ICS }}+\underbrace{0}_{\text {ERBS }}+\underbrace{0}_{\text {ERCS }}$

In an ideal RF propagation channel, the received signal amplitude is inversely proportional with the phase velocity $\phi_{c}(t)$ or the product of $\omega_{c} \tau(t)$. The ideal received signal phase decreases linearly with the phase velocity $\phi_{c}(t)$ or the product of $\omega_{c} \tau(t)$. We can clearly see that that a correct estimation of the signal amplitude and phase depends on a correct estimation of both the signal time delay (i.e., or distance) and frequency.

Next, let us describe what happens to these parameters when we consider a realistic RF geolocation propagation channel model.

\subsection{Description of a realistic $R F$ geolocation propagation channel model}

The analytical expression of the received RF GPS (or GNSS) signal, $u^{s}(t)$, which is a function of $\left(A^{s}, \phi^{s}, \tau^{s}, \omega^{s}\right)$ and has undergone propagation through the ionosphere, troposphere (all of which are included, later on, and of the multipath model) can be written as a product of two independent signals: received $\mathrm{BS}$ (or RBS), $u_{1}^{s}=\underbrace{A_{1}^{s} e^{j \phi_{1}^{s}}}_{\mathrm{RBS}}$, and received CS or (RCS),

$$
\begin{aligned}
& \underbrace{u_{2}^{S}(t)=A_{2}^{S}(t) \mathrm{e}^{j\left[\phi_{2}^{s}(t)+\omega^{s}\left(t_{r}\right)\right]}}_{\text {RCS }}, \\
& u^{S}(t)=\underbrace{A_{1}^{S} e^{j \phi_{1}^{S}}}_{\mathrm{RBS}} \underbrace{A_{2}^{S}(t) \mathrm{e}^{j\left[\phi_{2}^{S}(t)+\omega^{S}\left(t_{r}\right)\right]}}_{\mathrm{RCS}} \\
& =\underbrace{u_{1}^{s}}_{\text {RBS }} \underbrace{u_{2}^{s}(t)}_{\text {RCS }}
\end{aligned}
$$

Where 


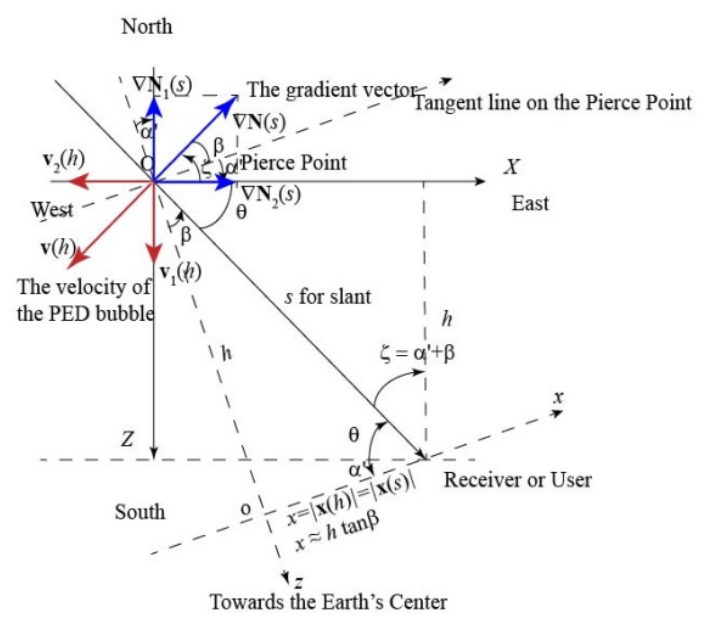

Fig. 9. Description of the ionospheric gradient vector, its components, and tangent line on the Pierce Point.

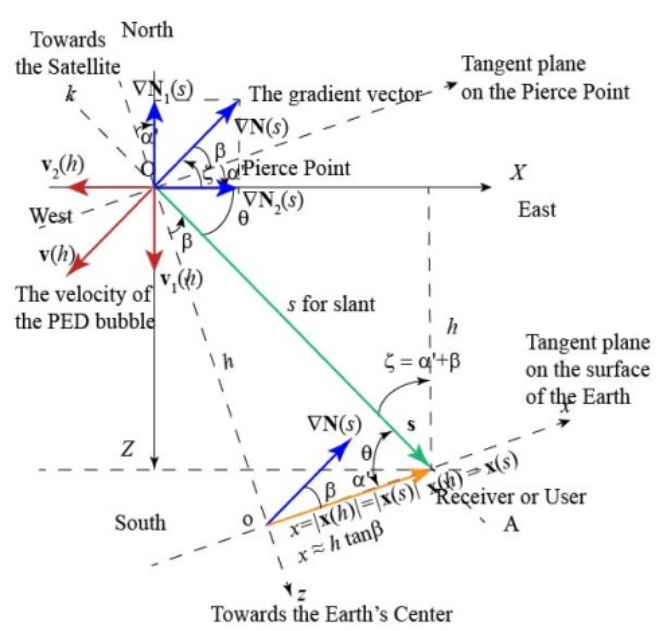

Fig. 10. Description of the ionospheric gradient vector, its components, and tangent plane on the Pierce Point and the surface of the Earth.

$t_{r}=t-\frac{\rho}{c}$

On the other hand, the received RF GPS (or GNSS) signal, $u^{s}(t)$, can also be expressed as a product of signal amplitude,

$A^{s}(t)$, and exponential phase, $e^{j\left[\phi^{s}\left(t ; \omega^{s}, \tau^{s}\right)+\omega^{s}\left(t-\frac{\rho}{c}\right)\right]}$, as follows

$u^{s}(t)=A^{s}(t) \mathrm{e}^{j\left[\phi^{s}(t)+\omega^{s}\left(t_{r}\right)\right]}$

Since (17) and (19) really represent the same thing equating both sides we obtain

$$
\begin{aligned}
& A^{s}(t)=A_{1}^{s} A_{2}^{s}(t) \\
& \phi^{s}(t)=\phi_{1}^{s}+\phi_{2}^{s}(t) \\
& \rho=R+c D_{e}^{s}
\end{aligned}
$$

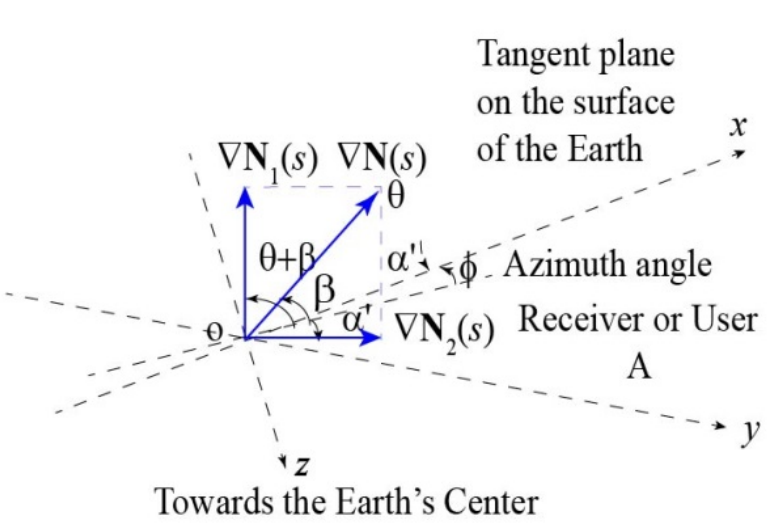

Fig. 11. Zoom of the tangent plane on the surface of the Earth.

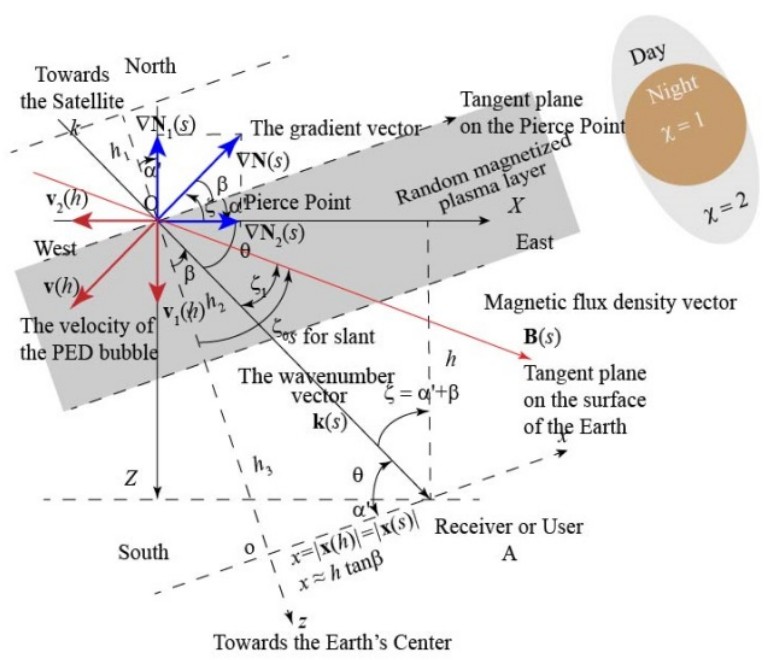

Fig. 12. Same as Fig. 10. Add the description of the random magnetized plasma layer and magnetic density flux vector.

$$
\begin{aligned}
& \text { Or } \\
& \tau^{s}=\tau+D_{e}^{s} \Rightarrow D_{e}^{s}=\tau^{s}-\tau \\
& \omega^{s}=\omega_{c}+\omega_{e}^{s}
\end{aligned}
$$

Which is equivalent with

$\omega_{e}^{s}=\omega^{s}-\omega_{c}=2 \pi f_{c}\left(\frac{c d D_{e}^{S}}{\lambda_{c} d t}=f_{e}^{s}\right)=\omega_{c} f_{e}^{s}$

Although (20) and (21) are an accurate description of the received RF GPS (or GNSS) signal, $u^{s}(t)$, amplitude, $A^{s}(t)$, and phase, $\phi^{s}(t)$, they do not show the relationship between the ideal transmitted signal, $s(t)$, and the received signal, $u^{s}(t)$. In order to accomplish that we need to express the received RF GPS (or GNSS) signal, $u^{s}(t)$, as a product of the ideal received signal (or IRS), $u_{0}(t)$, which is a function of $\left(A, \varphi, \tau, \omega_{c}\right)$, and environmental received signal (or ERS), $\delta u_{e}^{s}(t)$, which is a function of $\left(\delta A_{e}^{S}, \delta \phi_{e}^{s}, D_{e}^{S}, \omega_{e}^{s}\right)$, as follows 
$u^{s}(t)=\underbrace{u_{0}(t)}_{\mathrm{IRS}} \underbrace{\delta u_{e}^{S}(t)}_{\mathrm{ERS}}$

Where

$\delta u_{e}^{S}(t)=\delta A_{e}^{S}\left(t ; D_{e}^{S}, \omega_{e}^{S}\right) \mathrm{e}^{j\left[\delta \phi_{e}^{S}\left(t ; D_{e}^{S}, \omega_{e}^{S}\right)+\omega_{e}^{S}\left(t-D_{e}^{S}\right)\right]}$

$\delta A_{e}^{s}\left(t ; D_{e}^{s}, \omega_{e}^{s}\right)=A^{s}\left(t ; \tau^{s}, \omega^{s}\right) A\left(t ; \tau, \omega_{c}\right)^{-1}$

$\delta \phi_{e}^{s}\left(t ; D_{e}^{s}, \omega_{e}^{s}\right)+\omega_{e}^{s}\left(t-D_{e}^{s}\right)=\mid \begin{gathered}\phi^{s}\left(t ; \tau^{s}, \omega^{s}\right)+\omega^{s}\left(t-\frac{\rho}{c}\right) \\ -\underbrace{\phi_{0}}_{\text {IRBS }}-\underbrace{\omega_{c}(t-\tau)}_{\text {IRCS }}\end{gathered}$

$$
=\mid \begin{aligned}
& \phi^{s}\left(t ; \tau^{s}, \omega^{s}\right)-\underbrace{\phi_{0}}_{\text {IRBS }}+ \\
& \omega^{s} t-\underbrace{\omega_{c} t}_{\text {IRCS }}-\frac{\rho}{c}+\underbrace{\frac{R}{c}}_{\text {IRCS }}
\end{aligned}
$$

The description of the ERS is still not enough so we will need to further separate the ERS, $\delta u_{e}^{s}(t)$, into two components as follows

$$
\underbrace{\delta u_{e}^{s}(t)}_{\text {ERS }}=\underbrace{\delta u_{e 1}^{s}(t)}_{\text {ERBS }} \underbrace{\delta u_{e 2}^{s}(t)}_{\text {ERCS }}
$$

where

$$
\begin{aligned}
& \underbrace{\delta u_{e 1}^{S}(t)}_{\text {ERBS }}=\underbrace{\delta A_{e 1}^{S}(t)}_{\text {ERBS }} \underbrace{e^{j \delta \phi_{e 1}^{S}(t)}}_{\text {ERBS }} \\
& \underbrace{\delta u_{e 2}^{S}(t)}_{\text {ERCS }}=\underbrace{\delta A_{e 2}^{S}(t)}_{\text {ERCS }} \underbrace{e^{j\left[\delta \phi_{e 2}^{S}\left(t ; D_{e}^{S}, \omega_{e}^{S}\right)+\omega_{e}^{S}\left(t-D_{e}^{S}\right)\right]}}_{\text {ERCS }}
\end{aligned}
$$

Finally, in a realistic RF channel we should be able to precisely know (or estimate) the four "independent" iii signal parameters: $A^{s}(t), \phi^{s}(t), \tau^{s}(t)$, and $\omega^{s}(t)$ as follows:

$$
\begin{aligned}
& A^{S}(t)=\underbrace{A(t)}_{\mathrm{IRS}} \underbrace{\delta A_{e}^{S}\left(t ; D_{e}^{S}, \omega_{e}^{S}\right)}_{\mathrm{ERS}} \\
& =\underbrace{A_{0}}_{\text {IRBS }} \underbrace{\delta A_{0}(t)}_{\text {IRCS }} \underbrace{\delta A_{e 1}^{S}(t)}_{\text {ERBS }} \underbrace{\delta A_{e 2}^{S}\left(t ; D_{e}^{S}, \omega_{e}^{S}\right)}_{\text {ERCS }} \\
& =\underbrace{A_{\text {IRBS }}^{A_{0}} \underbrace{\delta A_{e 1}^{S}(t)}_{\text {ERBS }} \underbrace{\delta A_{0}\left(t ; \tau, \omega_{c}\right)}_{\text {RES }} \underbrace{\delta A_{e 2}^{S}\left(t ; D_{e}^{S}, \omega_{e}^{S}\right)}_{\text {IRCS }}}_{\text {RBS }} \\
& \phi^{S}(t)=\underbrace{\varphi(t)}_{\mathrm{IRS}}+\underbrace{\phi_{e}^{S}\left(t ; D_{e}^{S}, \omega_{e}^{S}\right)}_{\mathrm{ERS}} \\
& =\underbrace{\varphi_{1}}_{\text {IRBS }}+\underbrace{\varphi_{2}\left(t ; \tau, \omega_{c}\right)}_{\text {IRCS }}+\underbrace{\delta \phi_{e 1}^{s}(t)}_{\text {ERBS }}+\underbrace{\phi_{e 2}^{S}\left(t ; D_{e}^{S}, \omega_{e}^{S}\right)}_{\text {ERCS }} \\
& =\underbrace{\phi_{0}^{\phi_{0}}+\underbrace{\phi_{e 1}^{s}(t)}_{\text {ERBS }}}_{\text {RBS }}+\underbrace{\underbrace{\omega_{c}[t-\tau(t)]}_{\text {IRCS }}+\underbrace{\phi_{e 2}^{s}\left(t ; D_{e}^{s}, \omega_{e}^{s}\right)}_{\text {ERCS }}}_{\text {RCS }}
\end{aligned}
$$

$$
\tau^{s}(t)=\frac{\rho(t)}{c}
$$

$$
\begin{aligned}
& =\frac{R(t)+c D_{e}^{S}(t)}{c} \\
& =\underbrace{0}_{\text {IBS }}+\underbrace{0}_{\text {ERBS }}+\underbrace{\frac{R(t)}{c}}_{\text {ICS }}+\underbrace{D_{e}^{S}(t)}_{\text {ERCS }} \\
& =\underbrace{\tau(t)}_{\text {ICS }}+\underbrace{D_{e}^{S}(t)}_{\text {ERCS }} \\
\omega^{S}(t) & =\underbrace{\underbrace{\frac{d D_{e}^{S}(t)}{\lambda_{c}}}_{\text {ERBS }}[\underbrace{0}_{d t}[\underbrace{\left.\omega_{e}^{S}(t) \equiv 2 \pi f_{c} f_{e}^{S}(t)\right]}_{\text {IRCS }}}_{\text {IBS }} \\
& =\underbrace{2 \pi f_{c}\left[\equiv \omega_{c}\right]}_{\text {ERCS }} \underbrace{\left[1+f_{e}^{S}(t)\right]}_{\text {IRCS }}
\end{aligned}
$$

Equations (33) through (36) provide the entire description of the four most important "independent" signal parameters, $A^{s}(t), \phi^{s}(t), \tau^{s}(t)$, and $\omega^{s}(t)$, of a realistic RF propagation channel model.

In a realistic RF propagation channel, the realistic received signal amplitude is inversely proportional with the phase velocity $\phi_{c}(t)$ or the product of $\omega_{c} \tau(t)$ and it is a complicated function of the ERCS or $\left(D_{e}^{S}, \omega_{e}^{S}\right)$.

The received signal phase decreases linearly with the phase velocity $\phi_{c}(t)$ or the product of $\omega_{c} \tau(t)$ and it is also a complicated function of ERCS or $\left(D_{e}^{S}, \omega_{e}^{S}\right)$. We can clearly see that that a correct estimation of the signal amplitude and phase depends on a correct and complicated estimation of both the signal delay and frequency or their respective errors. It is not only important to estimate the signal delay error, $D_{e}^{S}$, but it is just as important to estimate the signal frequency error, $\omega_{e}^{s}$, which is the derivative of the signal delay error.

We should also mention that this is a first order approximation; i.e., $f_{e}^{s}(t)=d D_{e}^{s}(t) / d t$ is the only correction applied to the received carrier frequency. If this approximation is not good enough, based on verification of the fidelity of the model from real data, then later on the model can be enhanced to include second or higher order approximations. However, as we are going to see throughout the derivations of this paper, because the computation of $f_{e}^{s}(t)$ is in general very laborious, for the purposes of this paper, we are going to restrict all derivations based on this main assumption of the first order approximation. An example of higher order ionosphere errors is given by [24]. 
Next, we consider the description of ionospheric RF geolocation propagation channel model.

\section{Description of Ionospheric RF Geolocation Propagation Channel Model}

The description of the ionospheric RF geolocation propagation channel model contains the description of the ionospheric refractive index and refractivity, delay, Doppler frequency, and amplitude and phase scintillation.

\subsection{Ionospheric refractive index and refractivity}

Refractive index : In RF signal propagation, the refractive index or index of refraction, $n$, of the medium (or media or propagation channel or environment in our case the ionosphere) is a dimensionless number that describes how RF signals propagates through that medium.

In order to derive the refractive index for the ionospheric channel we make the following fundamental assumption. If we treat ionosphere as ionized gas as a perfect dielectric with relative permittivity [24], $\epsilon_{d}$, (and relative permeability [27] $\left.\mu_{d}=1\right)$ its refractive index [5], $n$, for a signal frequency, $f_{k}$, is defined as the ratio of $c$, the SOL [2] in vacuum (or in medium with refractive index [5], $n=1$ ), and $v$, the phase velocity or the SOL (or RF signals) in the medium (or ionosphere) with refractive index [5], $n \neq 1$, [28] (Progri 2019, [29])

$$
\begin{aligned}
n & =\frac{c}{v} \\
& =\sqrt{\underbrace{\mu_{d} \varepsilon_{d}}_{1}} \\
& =\sqrt{\underbrace{\varepsilon_{d r}+j \varepsilon_{d i}}_{\epsilon_{d}}} \\
& =\sqrt{\underbrace{1-j \frac{\sigma_{d}}{\varepsilon_{\varepsilon_{1} \omega_{k}}}}_{\epsilon_{d r}}} \\
\sigma_{d} & \cong-j \frac{\epsilon_{d i}}{m_{e} n_{e} \omega_{k}} \mathrm{iv} \\
\omega_{k} & =2 \pi f_{k}
\end{aligned}
$$

where (1) $\sigma_{d}$ is the dialectic conductivity of the ionosphere; (2) $e$ is the elementary charge [30]; (3) $m_{e}$ is the electron mass [30]; (4) $\varepsilon_{0}$ is the dielectric permittivity of vacuum [31]; and (5) $n_{e}$ the plasma [32] electron density (PED) in [els $\left.\cdot \mathrm{m}^{-3}\right] \mathrm{v}$

$$
\begin{aligned}
n & \cong \sqrt{1-\frac{e^{2}}{4 \pi^{2} m_{e} \varepsilon_{0}} \frac{n_{e}}{f_{k}^{2}}} \\
& \approx 1-\frac{1}{2} \frac{e^{2}}{4 \pi^{2} m_{e} \varepsilon_{0}} \frac{n_{e}}{f_{k}^{2}} \\
& =1-a \frac{n_{e}}{f_{k}^{2}} \\
& =1-\frac{f_{p}^{2}}{2 f_{k}^{2}} \mathrm{vi}
\end{aligned}
$$

(6) $f_{p}$ is the ionospheric plasma [32], [33] frequency [Hz]; with [30], [31], [34]-[40].

$$
\begin{aligned}
a & =\frac{k \cdot c}{A_{e} \cdot T E C} \\
& =\frac{10^{16} \cdot c^{2} \cdot r_{e}}{2 \pi} \cdot \frac{\mathrm{TECU} \cdot \mathrm{els}^{-1}}{\mathrm{TEC}} \\
& =\frac{10^{16} \cdot c^{2}}{2 \pi} \cdot \frac{1}{4 \pi \varepsilon_{0}} \frac{e^{2}}{m_{e} c^{2}} \cdot \frac{\mathrm{TECU} \cdot \mathrm{els}{ }^{-1}}{\mathrm{TEC}} \\
& =\frac{10^{16} \cdot \mathrm{TECU} \cdot \mathrm{els}}{\mathrm{TEC}} \cdot \frac{e^{2}}{8 \pi^{2} m_{e} \varepsilon_{0}} \\
& =\frac{\mathrm{els}{ }^{-1} e^{2}}{8 \pi^{2} m_{e} \varepsilon_{0}} \\
& =\frac{C_{x}}{2} \mathrm{vii}^{-1} \\
& =\frac{\mathrm{els}{ }^{-1} \cdot\left(1.602176565 \times 10^{-19} \mathrm{C}\right)^{2}}{8 \pi^{2} \cdot 9.10938291 \times 10^{-31} \mathrm{~kg} \cdot 8.854187817620 \times 10^{-12} \mathrm{CV}^{-1} \mathrm{~m}^{-1}} \\
& =40.308193030515824 \frac{\mathrm{els}^{-1} \mathrm{CVm}}{\mathrm{kg}} \mathrm{viii}^{1}
\end{aligned}
$$

(7) $a=40.308193030515824\left[\mathrm{els}^{-1} \mathrm{~m}^{3} \mathrm{~s}^{-2}\right]$ (see (41)) Equations (38) and (41) show that the refractive index [5], $n$, is a function of the PED, $n_{e}$ in $\left[\mathrm{els} \cdot \mathrm{m}^{-3}\right]$.

Note that the refractive index [5], $n$, is smaller than unity and strongly depends on the signal frequency, $f_{k}$. Thus, electromagnetic signals traversing through the ionosphere [2] are "depressively" delayed along their path, depending on the number of free electrons [28], [41].

Refractivity: The deviation of the refractive index [5], $n$, in the ionosphere (and troposphere or atmosphere) [2] from unity is tiny (a few parts per thousand [5]) under normal atmospheric conditions; thus, the scaled-up refractivity, $N$, is often used, (see (42)); i.e., [42], [43]

$N=(n-1) \times 10^{6} \approx-a \frac{n_{e}}{f_{k}^{2}} \times 10^{6}=-\frac{f_{p}^{2}}{2 f_{k}^{2}} \times 10^{6}$

Both $n\left(f_{k}\right)$ and $N\left(f_{k}\right)$ are monotonic increasing functions with respect to the signal frequency, $f_{k}$ as $\forall f_{k} \in(0, \infty)$. First, we consider the limit values; i.e., 


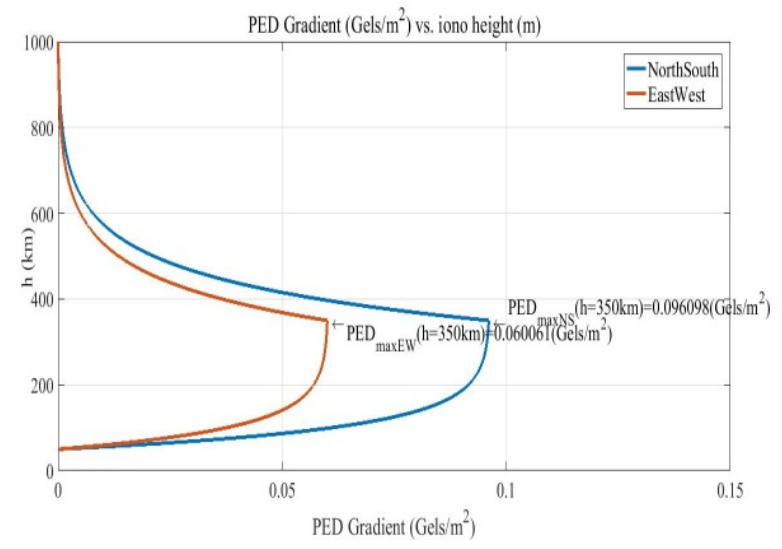

Fig. 13. First profile of the PED gradient $\left(\mathrm{Gels} / \mathrm{m}^{2}\right)$ vs. ionospheric height (m).

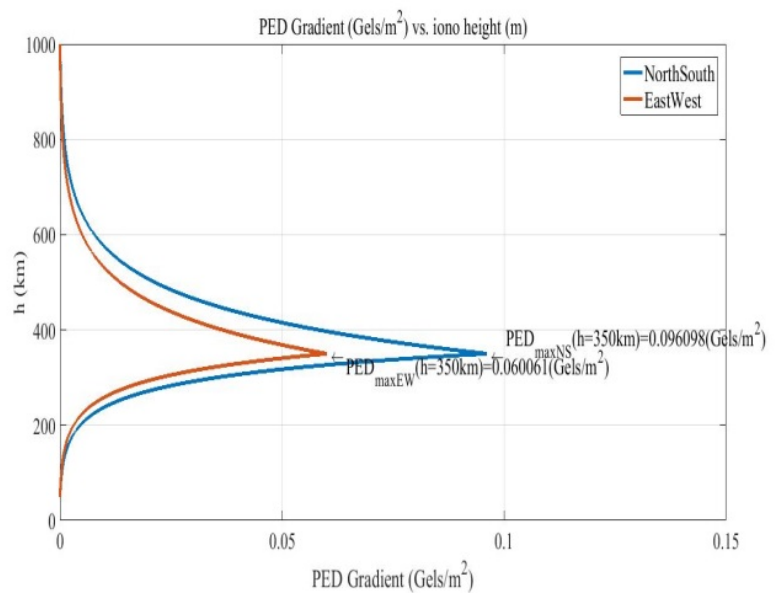

Fig. 14. Second profile of the PED gradient $\left(\right.$ Gels $\left./ \mathrm{m}^{2}\right)$ vs. ionospheric height (m).

$n\left(f_{k} \rightarrow 0\right)=-\infty ; n\left(f_{k} \rightarrow \infty\right)=1$

Which results in the following values of the refractivity

$N\left(f_{k} \rightarrow 0\right)=-\infty ; N\left(f_{k} \rightarrow \infty\right)=0$

Since, RF GNSS L5, L2, L3, and L1 signal frequency fall in the RF L-band range of $\forall f_{k} \in\left[f_{c 0}=1 \mathrm{GHz}, f_{c 1}=2 \mathrm{GHz}\right]$ then for most practical purposes, typical values of refractive index and refractivity are as follows,

$n\left(f_{c 0}=1 \mathrm{GHz}\right)=n_{c 0} \cong 1+N_{c 0} \times 10^{-6}$

$n\left(f_{c 1}=2 \mathrm{GHz}\right) \cong n_{c 1} \cong 1+N_{c 1} \times 10^{-6}$

$N\left(f_{c 0}=1 \mathrm{GHz}\right) \cong N_{c 0} \cong-40$

$N\left(f_{c 1}=2 \mathrm{GHz}\right) \cong N_{c 1} \cong-10$

Below a certain frequency $\left(f_{c 1}<1 \mathrm{GHz}\right)$, called the critical frequency $f_{c 0}=[5-898.026]^{\mathrm{ix}} \mathrm{MHz}$, the ray cannot cross the ionosphere [2], [44] for any elevation angle. Hence, for most practical purposes it is safe to assume that the GNSS RF L-band signals will be able to penetrate the ionosphere and the received GNSS RF L-band signals will be bent as a result of their propagation through the ionosphere by refractive index and

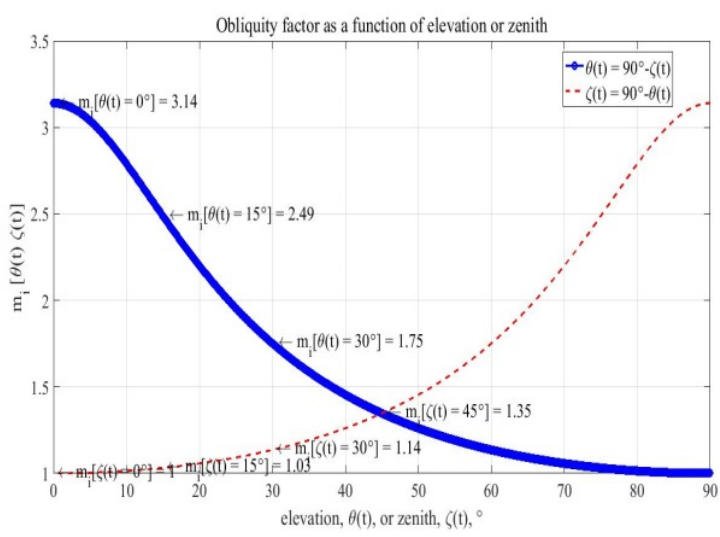

Fig. 15. Obliquity factor, $m_{i}(\zeta)$ or $m_{i}(\theta)$, as a function of the zenith, $\zeta$, or elevation, $\theta$, in $\left(^{\circ}\right)$.

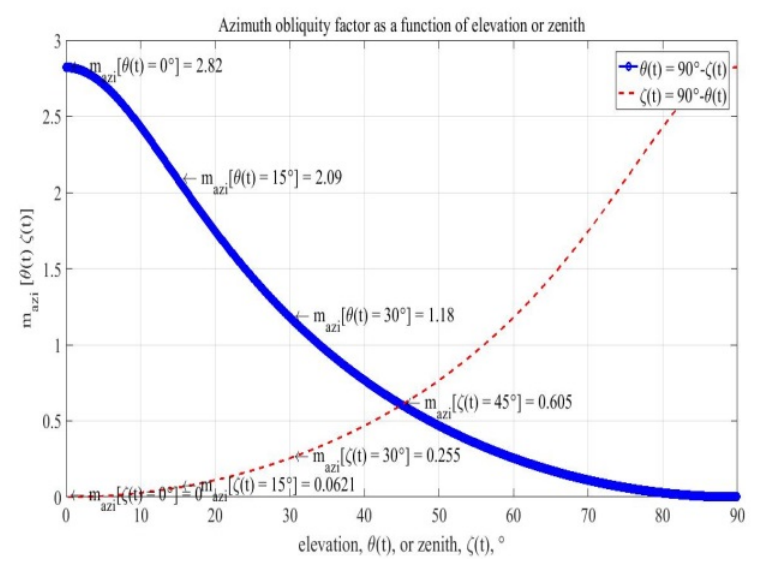

Fig. 16. Azimuth obliquity factor, $m_{i}^{a z}(\zeta)$ or $m_{i}^{a z}(\theta)$, as a function of the zenith, $\zeta$, or elevation, $\theta$, in $\left(^{\circ}\right)$.

refractivity values given by (42)-(48) respectively.

This concludes the description of the ionospheric refractive index and refractivity. Next, we consider the description of the Ionospheric delay.

\subsection{Description of the Ionospheric delay}

Ionospheric delay : It is the amount of additional transmission (or propagation) time a signal incurs as it passes through the ionosphere [11], [12]. The amount of delay through the ionosphere varies with the frequency of the signal that passes through the ionosphere, solar activity which influences the electron (and/or ions) content or PED of the ionosphere, time of day, day of the year, year of the millennium, elevation or zenith angles etc.

In general, this delay, $D_{i, t}^{S}(t)^{\mathrm{x}}$, is defined as the difference between the actual path of the carrier $S$ and the straight-line path in a vacuum $L^{\mathrm{xi}}$

$D_{i, t}^{S}(t)=\underbrace{\int_{S} \frac{d S}{v}}_{S}-\underbrace{\int_{l} \frac{d L}{c}}_{L} \rightarrow$

$c D_{i, t}^{s}=\int_{s} n d s-\int_{l} d l$ 


$$
=\underbrace{\int_{s}(n-1) d s}_{(1)}+\underbrace{\left\{\int_{s} d s-\int_{l} d l\right\}}_{(2)}
$$

In terms of distance, after multiplying by the SOL [2], $c$, gives two components: $(1) \equiv$ change of refractive delay along path length and (2) $\equiv$ change of path length [45].

Because the change of path length must be independent of speed then the following must hold

$\underbrace{\left\{\int_{s} d s-\int_{l} d l\right\}}_{(2)}=0$

Hence,

$c D_{i, t}^{s}=\underbrace{\int_{s} n d s-\int_{l} d l=\int_{s}(n-1) d s}_{(1)}=10^{-6} \int_{s} N d s$

Up until now the atmosphere was considered to be horizontally layered and azimuthally symmetric; i.e., refractive index (or refractivity) of the ionosphere is constant (or does not vary) as a function of horizontal position; it only varies as a function of height. Since the refractive index (or refractivity) of the ionosphere is not constant, but varies gradually with both height and horizontal position, ionosphere is known as a gradient-index or GRIN medium and is described by gradient index or azimuthally asymmetric mathematics (see Figs. 9-12) [5], [48], [49]. Although for most applications this assumption is appropriate, the azimuthally asymmetric condition may introduce significant errors in geodetic measurements where high precision is required to eliminate deep signal fading effects due to ionospheric scintillation [46] and ionospheric plasma bubble. The azimuthally asymmetric condition is described as a tilted atmosphere; whose refractivity $N$ as function of height $h$ and horizontal position $\mathbf{x}$ reads: (where the gradient vector (or tensor [48], [49]), $\nabla \mathbf{N}$, is defined as below (see Figs. 9-12)):

$N(\mathbf{x}, s)=N(0, s)+\left[\left.\nabla \mathbf{N}(s) \equiv \frac{\partial N(\mathbf{x}, s)}{\partial \mathbf{x}(s)}\right|_{\mathbf{x}=0}\right] \cdot \mathbf{x}(s)^{\mathrm{xii}}$

The total ionospheric delay [11] in an azimuthally asymmetric atmosphere is given by integrating both sides of (54) as follows

$D_{i}^{a s}(\zeta)=10^{-6} \int_{0}^{\infty}[N(\mathbf{x}, s) \equiv N(0, s)+\nabla \mathbf{N}(s) \cdot \mathbf{x}(s)] d s$

Where the component of (54) can be written as: $D_{i}^{S}(\zeta)$, is the azimuthally symmetric component of the total ionospheric delay

$D_{i}^{s}(\zeta)=10^{-6} \int_{0}^{\infty} N(0, s) d s$

And, $D_{i}^{a z}(\zeta)$, is the azimuthally asymmetric component of the same
$D_{i}^{a z}(\zeta)=10^{-6} \int_{0}^{\infty} \nabla \mathbf{N}(s) \cdot \mathbf{x}(s) d s$

Hence,

$D_{i}^{a s}(\zeta)=D_{i}^{s}(\zeta)+D_{i}^{a z}(\zeta)$

The vector $\mathbf{x}(s)$ is in the projected slant direction (projection on the surface) (see Figs. 9-12). Because we have the slant vector equal to

$$
\begin{aligned}
\mathbf{s} & \approx m_{i}\left(90^{\circ}-\zeta\right) \mathbf{x}(s) \\
& =m_{i}(\theta) \mathbf{x}(s) \\
& =\frac{1}{\cos (\beta)} \mathbf{x}(s)
\end{aligned}
$$

And the differential of the slant as

$$
\begin{aligned}
d s & \approx m_{i}(\zeta) d h \\
& =d h / \sin (\beta)
\end{aligned}
$$

hence, the absolute value of $\mathbf{x}(s)$ is given by

$$
\begin{aligned}
|\mathbf{x}(h)| & =|\mathbf{x}(s)| \\
& \approx \frac{m_{i}(\zeta) h}{m_{i}\left(90^{\circ}-\zeta\right)} \\
& =\tan \left(\zeta^{\prime}\right) h
\end{aligned}
$$

Hence, vector $\mathbf{x}(s)$ is approximately given by

$\mathbf{x}(s) \approx \frac{m_{i}(\zeta)}{m_{i}\left(90^{\circ}-\zeta\right)} \mathbf{x}(h) h$

First, substituting (59) into (55) yields, $D_{i}^{s}(\zeta)=10^{-6} m_{i}(\zeta) \int_{0}^{\infty} N(0, h) d h$

Second, substituting, (61) and (59) into (56) yields,

$D_{i}^{a z}(\zeta)=10^{-6} \frac{m_{i}^{2}(\zeta)}{m_{i}\left(90^{\circ}-\zeta\right)} \int_{0}^{\infty} \nabla \mathbf{N}(h) \cdot \mathbf{x}(h) h d h$

The typical range of variation for the ionospheric delay is very dependent on the frequency but it can move from less than one meter to more than one hundred meters. In general, the $D_{i}^{S}(\zeta)$ integrated along a path joining a satellite $k$ and a receiver $A$ can be split up into two contributions: vertical TEC and slant function, in the following way

$D_{i}^{S}(\zeta)=m_{i}(\zeta) D_{i}^{Z}=D_{i}^{Z}+\Delta_{i}^{a z}(\zeta)$

where $D_{i}^{Z}$ is the delay due to vertical TEC

$D_{i}^{Z}=10^{-6} \int_{0}^{\infty} N(0, h) d h \cong \pm \frac{a \cdot T E C}{f_{k}^{2}}$

and $\Delta_{i}^{a Z}(\zeta)$ is the slant component

$\Delta_{i}^{a z}(\zeta)=D_{i}^{z}\left[m_{i}(\zeta)-1\right]$

The dot in (63) denotes an inner product [48]. The 


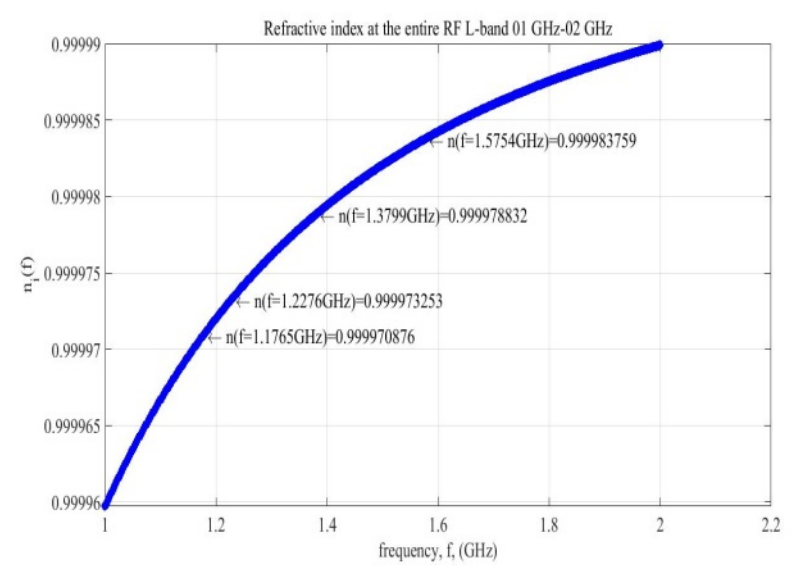

Fig. 17. Refractive index, $n_{i}(f)$, of the entire RF L band 1-2 GHz.

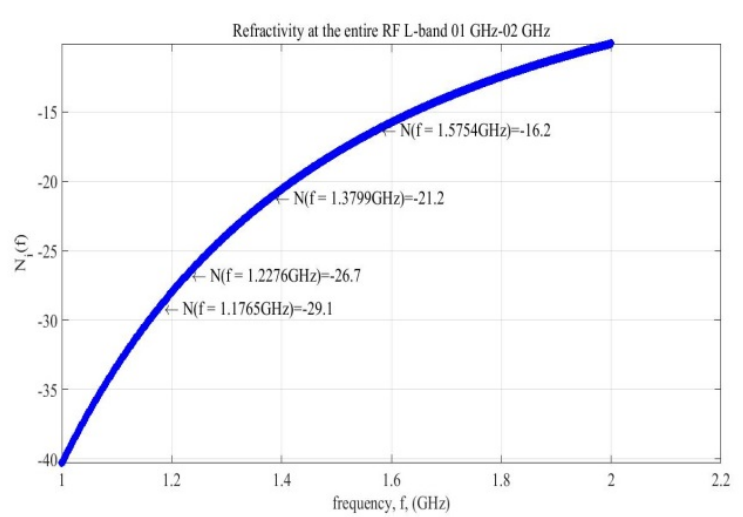

Fig. 18. Refractivity, $N_{i}(f)$, of the entire RF L band 1-2 GHz.

ionospheric delay [11] in an azimuthally asymmetric atmosphere can be given as the sum of the delay we would have in a symmetric atmosphere and a correction term due to the azimuthally asymmetric ionosphere.

The ionospheric obliquity factor $m_{i}[\theta(t)]$ (or $m_{i}[\zeta(t)]$ ) given by (85) or (86), on the other hand, is independent of the frequency but it is monotonic decreasing/increasing with elevation, zenith $\theta(t), \zeta(t) \in\left[0^{\circ}, 90^{\circ}\right] \quad$ (or $\zeta(t)=90^{\circ}-$ $\theta(t))$ as shown in Fig. 15. The ionospheric azimuth obliquity factor $m_{i}^{a z}[\zeta(t)]$ (or $m_{i}^{a z}[\theta(t)]$ ) given by (83) or (84), on the other hand, is independent of the frequency but it is monotonic decreasing/increasing with elevation, zenith $\theta(t), \zeta(t) \in$ $\left[0^{\circ}, 90^{\circ}\right]\left(\right.$ or $\left.\zeta(t)=90^{\circ}-\theta(t)\right)$ as shown in Fig. 16.

The correction term follows from (63) as

$D_{i}^{a z}(\zeta) \approx \frac{m_{i}^{2}(\zeta)}{m_{i}(\theta)}\left[\frac{\int_{0}^{\infty} \nabla \mathbf{N}(h) \cdot \mathbf{x}(h) h d h}{10^{6}}=\mathbf{G}_{i} \cdot \mathbf{e} \equiv G_{i}(\phi)\right]$

and $D_{i}^{a z}(\zeta)$ can be approximated as in the right-hand side of (62); $\mathbf{e}=[\cos \phi ; \sin \phi]$ is a unit vector in the direction of $\mathbf{x}(h)$; hence, we obtain

$\mathbf{G}_{i}=10^{-6} \int_{h_{0}}^{\infty} \nabla \mathbf{N}(h) h d h$

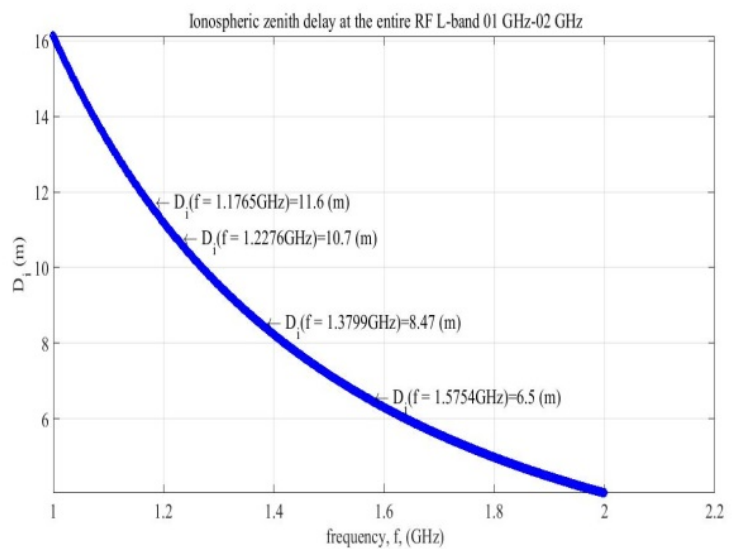

Fig. 19. Ionospheric zenith delay, $D_{i}^{z}(f)(\mathrm{m})$, of the entire RF L band 1-2 $\mathrm{GHz}$.

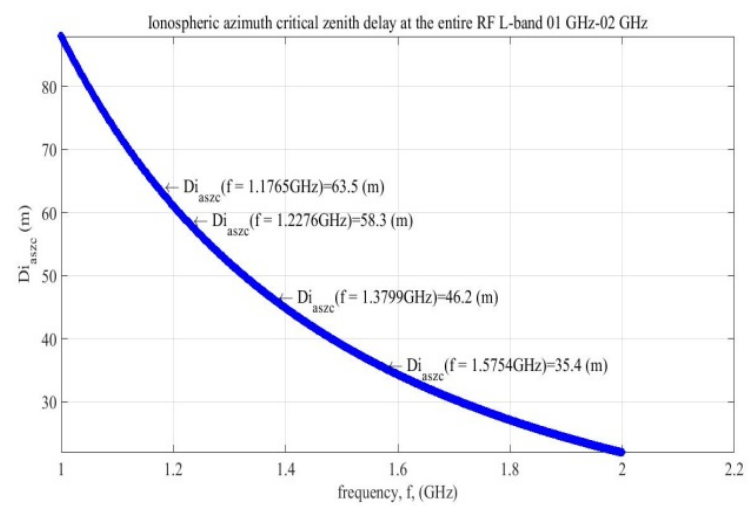

Fig. 20. Ionospheric zenith critical delay, $D_{i}^{a z, z c}(f)(\mathrm{m})$, of the entire RF L band 1-2 GHz.

Which is equivalent with

$\mathbf{G}_{i}=-\frac{a\left[\int_{h_{0}}^{\infty} \nabla \mathbf{n}_{\mathbf{e}}(h) h d h \equiv \sum_{l=1}^{2} \int_{h_{0}}^{\infty} v_{l}(h) h d h \mathbf{e}_{l}\right]}{f_{k}^{2}}$

where the expression of $v_{l}(h)$ can be obtained from

$v_{l}(h)=\left.\frac{\partial n_{e}(h)}{\partial e_{l}}\right|_{e_{l}=0}, l=\{1 \equiv N S, 2 \equiv E W\}$

where $\mathbf{v}(h)=\left\{v_{l}(h)\right\}, \quad l=\{1 \equiv N S, 2 \equiv E W\}$, is the velocity of the PED bubble with components in the NorthSouth and East-West directions and $\nabla \mathbf{n}_{\mathbf{e}}(h)$ is the gradient vector of the $n_{e}(h)$ in the opposite direction of the projected normal with components

$$
\begin{aligned}
\nabla \mathbf{n}_{\mathbf{e}}(h) & \equiv \mathbf{v}(h) \\
& =\left[\left.\frac{\partial n_{e}(h)}{\partial e_{l}}\right|_{e_{l}=0}=v_{l}(h)\right]_{l=\{1 \equiv N S, 2 \equiv E W\}}
\end{aligned}
$$

If we further define

$$
c_{l}=\left.\frac{\partial T E C}{\partial e_{l}}\right|_{e_{l}=0}
$$




$$
\begin{aligned}
& =\left.\int_{h_{0}}^{\infty} \frac{\partial n_{e}(h)}{\partial e_{l}}\right|_{e_{l}=0} h d h \\
& =\int_{h_{0}}^{\infty} v_{l}(h) h d h
\end{aligned}
$$

Then if we substitute (72) into (69) the following is obtained

$$
\begin{aligned}
\mathbf{G}_{i} & =-\frac{a \int_{h_{0}}^{\infty} \nabla \mathbf{n}_{\mathbf{e}}(h) h d h}{f_{k}^{2}} \\
& =-\frac{a \nabla \mathbf{T E C}}{f_{k}^{2}} \\
& =-\frac{a \sum_{l=1}^{2} c_{l} \mathbf{e}_{\mathbf{l}}}{f_{k}^{2}}
\end{aligned}
$$

is the gradient vector in the opposite direction of the projected normal with components $\mathbf{G}_{i}=\left\{G_{i, l}\right\}, l=\{1 \equiv N S, 2 \equiv E W\}$.

The first part of (67), $\frac{m_{i}^{2}(\zeta)}{m_{i}(\theta)}$, is a (mapping) function of the zenith or elevation angles $(\zeta, \theta)$. The second part is the azimuth $(\phi)$ dependent gradient part (see Figs. 9-12)

$$
G_{i}(\phi)=\left[G_{i, 1} \equiv-\frac{a c_{1}}{f_{k}^{2}}\right] \cos (\phi)+\left[G_{i, 2} \equiv-\frac{a c_{2}}{f_{k}^{2}}\right] \sin (\phi)
$$

Furthermore since, azimuth $(\phi)$ is given by

$\tan (\phi)=\frac{\sin (\phi)}{\cos (\phi)}=\frac{G_{i, 1}}{G_{i, 2}} \rightarrow \phi=\tan ^{-1}\left(\frac{G_{i, 1}}{G_{i, 2}}\right)$

Furthermore, since

$$
\cos (\phi)=\frac{1}{\sqrt{1+\left[\tan (\phi) \equiv \frac{G_{i, 1}}{G_{i, 2}} \equiv \frac{c_{1}}{c_{2}} \equiv c_{12}\right]^{2}}} \equiv c_{\phi}
$$

and

$$
\sin (\phi)=\frac{1}{\sqrt{1+\left[\cot (\phi) \equiv \frac{G_{i, 2}}{G_{i, 1}} \equiv \frac{c_{2}}{c_{1}} \equiv c_{21}\right]^{2}}} \equiv s_{\phi}
$$

Hence, the final formula of the gradient, $G_{i}$, (or the critical azimuth zenith delay, $D_{i}^{a z, z}$ ) can be computed from

$$
G_{i} \equiv D_{i}^{a z, z c}(t)=\frac{2 a}{f_{k}^{2}} \mid \begin{aligned}
& \left(\frac{G_{i, 1} f_{k}^{2}}{a} \equiv-c_{1}\right) c_{\phi}, \quad \text { if } G_{i, 1} \leq G_{i, 2} \\
& \left(\frac{G_{i, 2} f_{k}^{2}}{a} \equiv-c_{2}\right) s_{\phi}, \quad \text { if } G_{i, 1} \geq G_{i, 2}
\end{aligned}
$$

Therefore, the azimuth delay can be computed from either the gradient or the critical azimuth zenith delay as follows

$D_{i}^{a z}(\zeta) \approx \frac{m_{i}^{2}(\zeta)}{m_{i}(\theta)} G_{i}=\frac{m_{i}^{2}(\zeta)}{m_{i}(\theta)} D_{i}^{a z, z c}$

Using the above given definition, a slant gradient delay is a product of a gradient MF $m_{i}^{a z}(\zeta)$ and gradient delay $G_{i}(\phi)$, as shown in Figs. 9-12

$D_{i}^{a z}(\zeta)=m_{i}^{a z}(\zeta) G_{i}(\phi) ; m_{i}^{a z}(\zeta)=\frac{m_{i}^{2}(\zeta)}{m_{i}(\theta)}$

In analogy to the symmetric case, the "asymmetric" delay in the slant direction is also a product of a MF and, in this case, the delay in the direction of the normal $D^{n} \approx D_{i}^{Z}$

$$
\begin{aligned}
D_{i}^{a s}(\phi, \zeta) & =m_{i}[\zeta-\epsilon \cos (\beta)] D^{n} \\
& \approx\left[m_{i}(\zeta)-\frac{d m_{t}(\zeta)}{d \zeta} \epsilon \cos (\beta)\right] D_{i}^{Z}
\end{aligned}
$$

where $\beta$ is the angle between the projected normal and the projected slant direction. The approximation is allowed because $\epsilon \cos (\beta)$ is small. From (57) and (81) now follows

$D_{i}^{a z}(\phi, \zeta)=-\left[\frac{d m_{i}(\zeta)}{d \zeta} \approx \sec (\zeta) \tan (\zeta)\right] \epsilon \cos (\beta) D_{i}^{Z}$

So, if the RF signal is received from the same direction as the normal, the total delay is the smallest, caused by a thinner atmosphere. Because the $\mathrm{MF}$ is approximately $m_{i}(\zeta) \approx$ $\sec (\zeta)$, and so $d m_{i}(\zeta) / d \zeta \approx \sec (\zeta) \tan (\zeta)$, from (67), (72), and (74) can be seen that $\mathbf{G}_{i} \cdot \mathbf{e}=\left|\mathbf{G}_{i}\right| \cos (\beta) \approx-D_{t}^{Z} \epsilon \cos (\beta)$. Therefore, the length of the gradient vector, $\left|\mathbf{G}_{i}\right|=D_{i}^{Z}|\epsilon|$ is about which is an upper bound of the gradient delay for a particular azimuth as shown in Figs. 9-12.

The azimuthal MF (AMF), $m_{i}^{a z}(\zeta)$ (or $m_{i}^{a z}(\theta)$ ), is given as

$m_{i}^{a z}(\zeta) \cong \frac{m_{i}^{2}(\zeta)}{m_{i}(\theta)}=\frac{\cos ^{2}\left(\theta^{\prime}\right)}{\cos \left(\zeta^{\prime}\right)}=\frac{\sin ^{2}\left(\zeta^{\prime}\right)}{\cos \left(\zeta^{\prime}\right)}$

$m_{i}^{a z}(\theta) \cong \frac{m_{i}^{2}(\theta)}{m_{i}(\zeta)}=\frac{\sin ^{2}\left(\zeta^{\prime}\right)}{\sin \left(\theta^{\prime}\right)}=\frac{\cos ^{2}\left(\theta^{\prime}\right)}{\sin \left(\theta^{\prime}\right)}$

Where

$$
\begin{aligned}
m_{i}(\zeta) & =\frac{1}{\sqrt{1-\varrho^{2}}} \\
& =\frac{1}{\cos \left(\sin ^{-1} \varrho\right)} \\
& =\frac{1}{\cos \left(\zeta^{\prime}\right)} \\
& =\sec \left(\zeta^{\prime}\right) \\
& \approx \frac{1}{\sqrt{1-0.948 \sin ^{2}(\zeta)}}
\end{aligned}
$$

$$
\begin{aligned}
m_{i}(\theta) & =\frac{1}{\sin \left(\theta^{\prime}\right)} \\
& =\csc \left(\theta^{\prime}\right)
\end{aligned}
$$




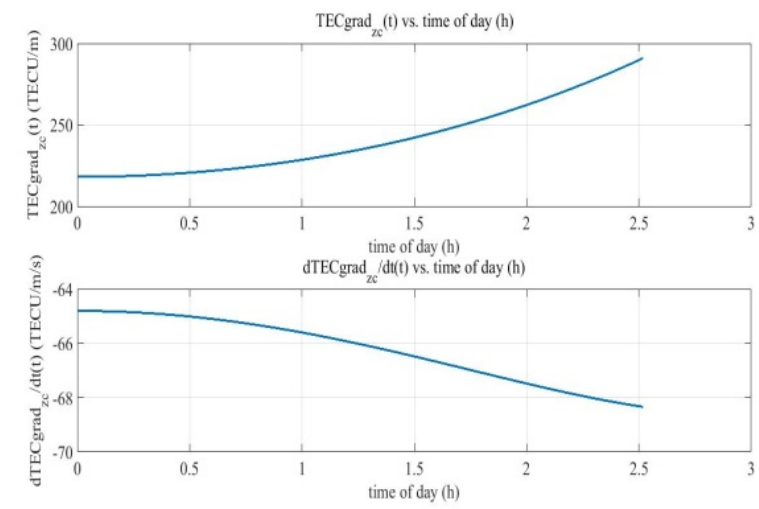

Fig. 21. TEC Gradient, $\frac{\partial T E C(x, t)}{\partial x}(\mathrm{TECU} / \mathrm{m})$ (top), and the derivative of the TEC Gradient, $\frac{d \frac{\partial T E C(x, t)}{\partial x}}{d t}$ vs. (TECU/m/s) (bottom), time of day (h) of the first profile. Reprinted with permission copyright (C) 2017 Ilir Progri.

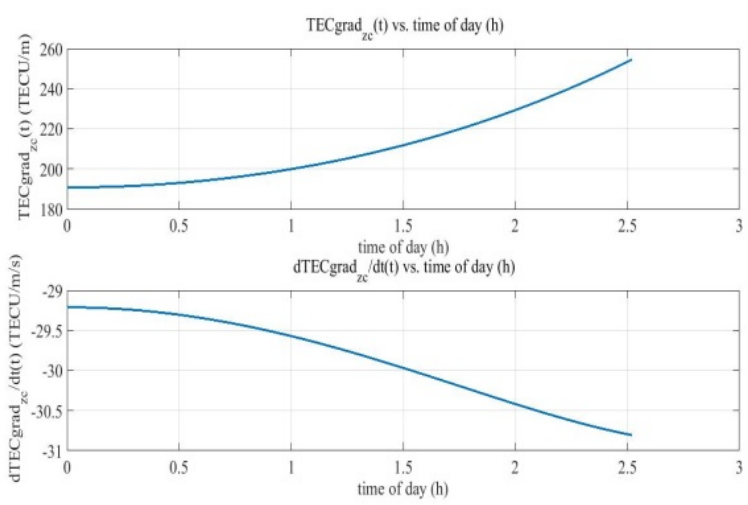

Fig. 22. TEC Gradient, $\frac{\partial \operatorname{TEC}(x, t)}{\partial x}(\mathrm{TECU} / \mathrm{m})$ (top), and the derivative of the TEC Gradient, $\frac{d \frac{\partial T E C(x, t)}{\partial x}}{d t}$ vs. (TECU/m/s) (bottom), time of day (h) of the second profile. Reprinted with permission copyright (C) 2017 Ilir Progri.

$$
\approx \frac{1}{\sqrt{1-0.948 \cos ^{2}(\theta)}}
$$

$\varrho=r\left\{\begin{array}{l}\cos (\theta) \\ \sin (\zeta)\end{array} ; r=\frac{R_{e}}{R_{e}+h_{i}}\right.$

$R_{e}$ is the mean radius of the Earth, typically, $R_{e}=6,371 \mathrm{~km}$ (see more [50]), $h_{i}=350 \mathrm{~km}^{\mathrm{xiii}}$ is the ionospheric shell ${ }^{\mathrm{xiv}}$ height, $\left\{\theta, \theta^{\prime}\right\}$ elevation angle of the $k$ th satellite at the user position and IP position (see Figs. 9-12) and $\left\{\zeta, \zeta^{\prime}\right\}$ are the zenith angles of the $k$ th satellite at the user position and IP position respectively [69]-[14]; hence,

$$
\begin{aligned}
D_{i}^{a z}(\zeta) & =10^{-6} \int_{h_{0}}^{\infty} \nabla \mathbf{N}\left(h_{0}\right) \cdot \mathbf{x} d s \\
& \approx \frac{\sin ^{2}\left(\zeta^{\prime}\right)}{\cos \left(\zeta^{\prime}\right)} \mathbf{G}_{i} \cdot \mathbf{e} \\
& =m_{i}^{a z}(\zeta) D_{i}^{a z, z c}(t)
\end{aligned}
$$

As can be seen from Figs. 13-16, gradients become

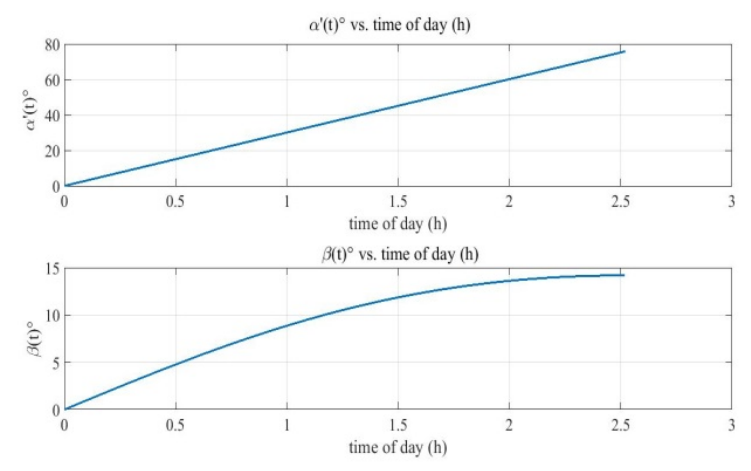

Fig. 23. The angle $\alpha^{\prime}(t)\left(^{\circ}\right)$ (top) and the angle $\beta(t)\left({ }^{\circ}\right)$ (bottom) as a function of time of day (h). Reprinted with permission copyright (C) 2017 Ilir Progri.

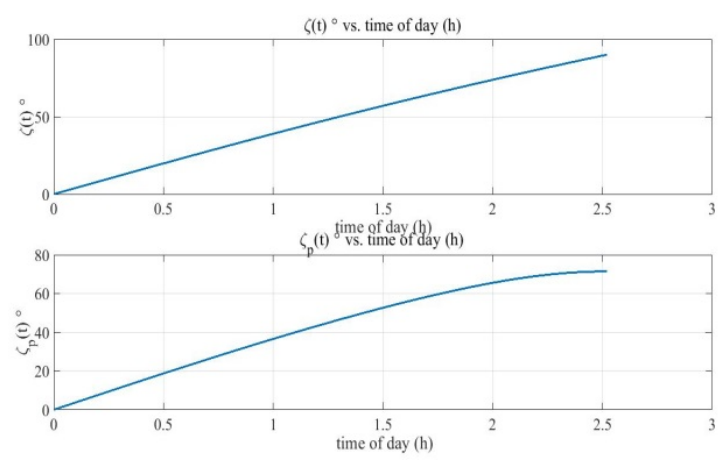

Fig. 24. The angle $\zeta(t)\left({ }^{\circ}\right)$ (top) and the angle $\zeta^{\prime}(t)\left(^{\circ}\right)$ (bottom) as a function of time of day (h). Reprinted with permission copyright (C 2017 Ilir Progri.

increasingly influential at lower elevation angles.

Another equivalent expression of the effects of azimuthally asymmetric, atmospheric delay is primarily caused by inhomogeneous PED distribution. $\mathcal{D}_{i}^{a z}(t)$ can be expressed as $\mathcal{D}_{i}^{a z}(t)=m_{i}^{a z}(\theta)\left(G_{i, 1} \cos \phi+G_{i, 2} \sin \phi\right)$

$$
=m_{i}^{a z}(\theta) D_{i}^{a z, z c}(t)
$$

where $D_{i}^{a z, z c}$ is the critical azimuth zenith delay corresponding to $\cos ^{2}\left(\theta^{\prime}\right) / \sin \left(\theta^{\prime}\right)=1$ or $\theta^{c}=41.8227^{\circ}$; i.e., for $0^{\circ} \leq$ $\theta \leq 41.8227^{\circ} \mathcal{D}_{i}^{a z}(\theta) \geq D_{i}^{a z, z c}$ and for $\theta>\theta^{c}=41.8227^{\circ}$ we have that $\mathcal{D}_{i}^{a z}(\theta)<D_{i}^{a z, z c}$.

Up to this point the derivation of the total ionospheric delay included only the influence of the azimuth, elevation (or zenith) and height of the ionosphere. Since experimental data has shown that PED varies considerably as a function of GNSS receivers' geodetic latitude, $\vartheta_{h}$, longitude, $\varphi_{h}$, and time, $t$, then the following expression of the zenith/slant ionospheric [2] delays and the ionospheric gradients along with MFs can be used to estimate the total asymmetrical ionospheric delay as [11], [62]-[66].

$D_{i}^{a s}(t)=D_{i}^{s y}(t)+D_{i}^{a z}(t)$

$\mathcal{D}_{i}^{a s}(t)=\mathcal{D}_{i}^{s y}(t)+\mathcal{D}_{i}^{a z}(t)$ 
Where

$D_{i}^{s y}(t)=m_{i}[\zeta(t)] D_{i}^{z}(t)$

$\mathcal{D}_{i}^{s y}(t)=m_{i}[\theta(t)] D_{i}^{z}(t)$

$D_{i}^{a z}(t)=m_{i}^{a z}[\zeta(t)] D_{i}^{a z, z c}(t)$

$\mathcal{D}_{i}^{a z}(t)=m_{i}^{a z}[\theta(t)] D_{i}^{a z, z c}(t)$

where $D_{i}^{a z} / \mathcal{D}_{i}^{a z}$ is the azimuth delay as a function of $\zeta(t) / \theta(t), \vartheta_{h}, \varphi_{h}$ and $t$ and $D_{i}^{a z, z c}$ is the critical zenith ionospheric horizontal gradient and $\zeta(t) / \theta(t)$ are the zenith and elevation angles of the IP point (see Figs. 9-12) [64], [67], [68].

The differential ranging error due to ionospheric spatial gradient was also modeled using the associated LAAS model [63], [65], [69]-[75] or L2C [76]

$$
\begin{aligned}
D_{i}^{a z}(\zeta) & =\frac{\frac{d D_{i}^{z}}{d x} x}{\sqrt{1-\varrho^{2}}} \\
& \cong\left(\frac{d D_{i}^{z}}{d x} \hat{x}+2 \tau v_{\text {air }}\right) m_{i}(\zeta)^{\mathrm{xv}} \\
\mathcal{D}_{i}^{a z}(\theta) & \cong\left(\frac{d D_{i}^{z}}{d x} \hat{x}+2 \tau v_{\text {air }}\right) m_{i}(\theta)
\end{aligned}
$$

where $D_{i}^{a z}(\theta), \mathcal{D}_{i}^{a z}(\theta)$ is the [ $L_{1}$ for example] ionospheric error (or azimuth delay, negative for carrier), $d D_{i}^{Z} / d x$ is the vertical ionospheric gradient; and $x_{k}$ (or $x_{\text {air }}$ ) is the distance (slant range) of the airplane from the ship (or the GBAS reference point $[\mathrm{m}]) ; \tau$ is the service type dependent time constant [8] of the smoothing filter and $v_{\text {air }}$ is the horizontal speed of the aircraft $[\mathrm{m} / \mathrm{s}]$.

Equations (96) or (97) provides the formula that is able to reconcile the expression not found anywhere else

$$
\begin{aligned}
\frac{d D_{i}^{z}}{d x} x & =\cot \left(\theta^{\prime}\right)\left[G_{i, 1} \cos (\phi)+G_{i, 2} \sin (\phi)\right] \\
& =\Delta_{i}^{a z}(\theta, \phi, t)
\end{aligned}
$$

And

$\frac{d D_{i}^{z}}{d x} x m_{i}(\zeta)=m_{i}^{a z}(\zeta) D_{i}^{a z, z c} \rightarrow$

$\frac{d D_{i}^{z}(t)}{d x}=\frac{m_{i}^{a z}(\zeta) D_{i}^{a z, z c}(t)}{x_{k} m_{i}(\zeta)}$

Substituting (80) into (100) the final expression for $D_{i}^{a z, z c}$ is obtained

$$
\begin{aligned}
D_{i}^{a z, z c}(t) & =\frac{m_{i}(\zeta)}{m_{i}^{a z}(\zeta)} \frac{d D_{i}^{Z}(t)}{d x} x \\
& =\frac{m_{i}(\theta)}{m_{i}(\zeta)} \frac{d D_{i}^{Z}}{d x} x
\end{aligned}
$$

$$
=\cot \left(\zeta^{\prime}\right) \frac{d D_{i}^{z}}{d x} x
$$

This concludes the description of the ionospheric delay.

\subsection{Description of the Ionospheric Doppler Frequency}

There appears to be at least three good references: Nickisch et al. in 2006, [77] in "Range rate - Doppler correlation for HF propagation in traveling ionospheric disturbance environments," and Zhou et al., 2012 [78] "Evidence of low-latitude daytime large-scale traveling ionospheric disturbances observed by high-frequency multistatic backscatter sounding system during a geomagnetically quiet period," who are directly linked with the ionospheric Doppler frequency estimation and Chum et al. 2012 [79] "Statistical investigation of horizontal propagation of gravity waves in the ionosphere over Europe and South Africa" which is indirectly liked with the ionospheric Doppler frequency estimation. Nickisch et al. in 2006, [77] claims: "Using ionospheric sounding together with fast computational inverse processing, it is now possible to obtain good real-time ionospheric models for use in geolocation for over-the-horizon (OTH) radar." Zhou et al. 2012 [78] claims: "Vertical phase velocity was also evaluated to be $<42 \mathrm{~m} / \mathrm{s}$ through the Doppler measurements." Chum et al. 2012 [79] claims: "Simultaneous measurements from nearby ionosonde made it possible to estimate the height of the ground waves (GW) observations and show that the analyzed GWs propagated at altitudes from $\sim 150$ to $\sim 250 \mathrm{~km}$. The analyzed waves were mainly observed after sunrise and around sunset. Our statistical study shows that the analyzed GWs propagated with horizontal velocities from $\sim 70$ to $250 \mathrm{~m} / \mathrm{s}$. The average observed horizontal velocities were $\sim 100 \mathrm{~m} / \mathrm{s}$ in the local summer and $125-250 \mathrm{~m} / \mathrm{s}$ in the local winter." According to Jandieri et al. 2009 [80], "Experimental investigations of Doppler shifts of ionospheric signal (basic frequency was $\sim 17 \mathrm{MHz}$ ) reflected from the ionosphere were carried out in Moscow State University, Moscow (Lat. 0 N, 0 55.5, Long. 0 E, 0 37.3) USSR in 1979. It was established that electron density fluctuations have a power-law spectrum with spectral index $3.8 \leq p \leq 4.6$, $\langle p\rangle=4.2$. Drift velocity of large-scale ionospheric irregularities is about $\sim 170 \mathrm{~m} / \mathrm{s}$ and corresponds to linear scales from several hundred to several tens of kilometers." Furthermore, horizontal mean drift velocity is $257 \mathrm{~m} / \mathrm{s}$ (day) and $185 \mathrm{~m} / \mathrm{s}$ (night); more frequently $136 \div 170 \mathrm{~m} / \mathrm{s}$ translate to ionospheric Doppler frequencies in the range of 40- 


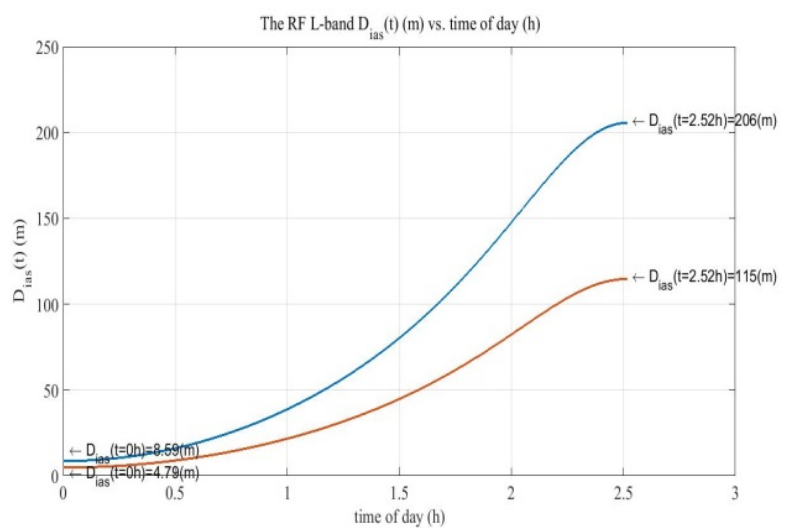

Fig. 25. Total ionospheric delay, $D_{i}^{a s}(t)(\mathrm{m})$, as a function of time of day (h) using the first profile.

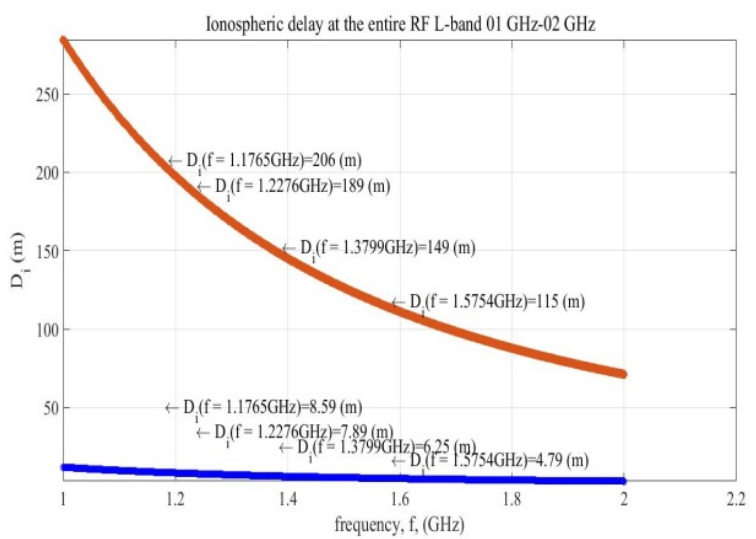

Fig. 26. Total ionospheric delay, $D_{i}^{a s}(t)(\mathrm{m})$, as a function of frequency $(\mathrm{GHz})$ using the first profile.

\section{$53 \mathrm{~Hz}$ at the GPS L1.}

However, the derivations in this section are original and unique neither based on any analytical treatise of Nickisch et al. in 2006, [77], nor Zhou et al. 2012 [78], and nor Chum et al. 2012 [79] which are specifically used for radar signal processing. Moreover, it is important to know that depending on the solar activity the Doppler frequency estimated from the theory presented in this section is in total agreement with 4parameter model theory presented in this paper.

The ionospheric Doppler frequency, $f_{i}^{D, a s}(t)$ or $f_{i}^{\mathcal{D}, a s}(t)$, is a subcomponent of the $f_{e}^{s}(t)$ or assuming that all other channel effects are ignored then

$$
f_{e}^{s}(t)=f_{i}^{D, a s}(t) \text { or } f_{i}^{\mathcal{D}, a s}(t) ; t \in[0, T)
$$

In this paper we are going to ignore the other channel components such as the tropospheric and multipath effects.

Nevertheless, before we can discuss the ionospheric Doppler frequency, we need to do some preliminary calculations.

Typically, a GPS satellite orbital period is one-half a sidereal day, i.e., 11 hours and 58 minutes or $T=718 \min$ or $T=$ $718 \times 60 \mathrm{~s}$. Hence, the angle $\alpha^{\prime}(t)$ as a function of time is as

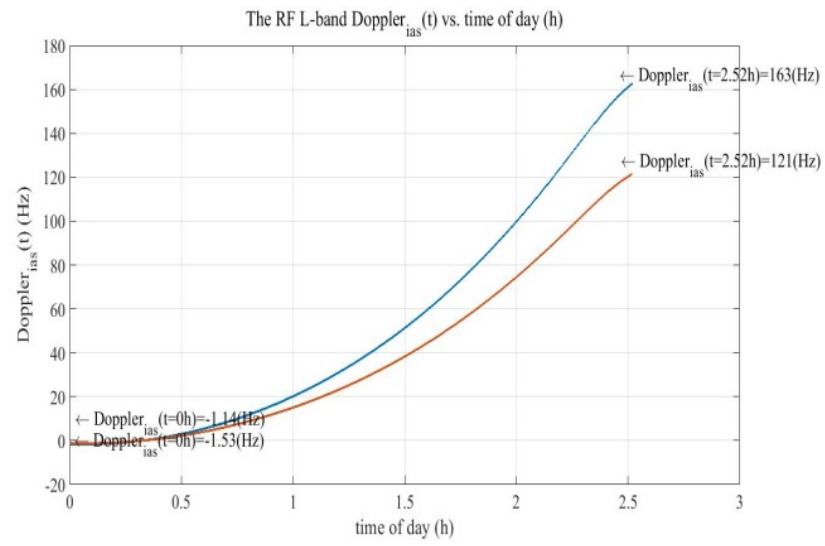

Fig. 27. Total ionospheric Doppler, $f_{i}^{D, a s}(t)(\mathrm{Hz})$, as a function of time of day (h) using the first profile.

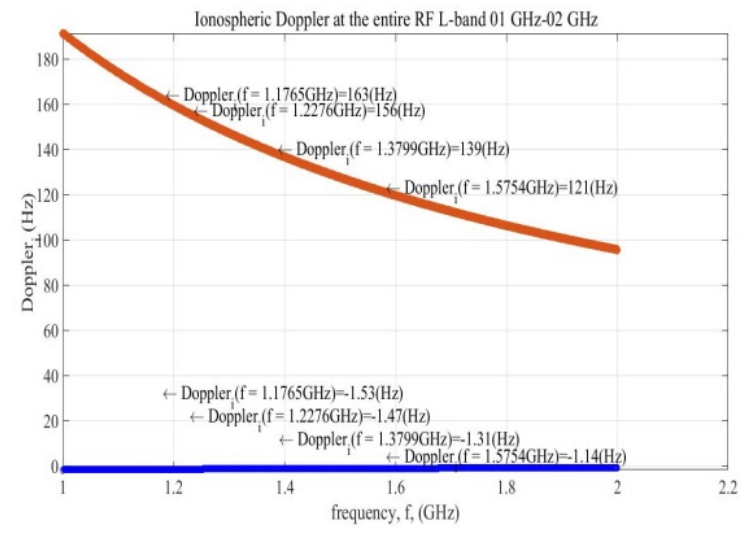

Fig. 28. Total ionospheric Doppler, $f_{i}^{D, a s}(t)(\mathrm{Hz})$, as a function of frequency $(\mathrm{GHz})$ using the first profile.

follows:

$\alpha^{\prime}(t)=\left(\frac{2 \pi \text { or } 360^{\circ}}{T} \equiv k_{\alpha^{\prime}}\right) t ; t \in[0, T)$

Next, we compute the angle $\beta(t)$ as follows:

$\beta(t)=\tan ^{-1}\left[\frac{R_{e} \sin \left[\alpha^{\prime}(t)\right]}{R_{S}-R_{e} \cos \left[\alpha^{\prime}(t)\right]}\right] ; t \in[0, T)$

where $R_{s}=26,600 \mathrm{~km}$ is the GPS satellite orbital radius.

The zenith angle, $\zeta(t)$, is the sum of two angles $\alpha^{\prime}(t)$ with $\beta(t)$ (see Figs. 9-12) as follows:

$\zeta(t)=\alpha^{\prime}(t)+\beta(t)=k_{\alpha^{\prime}} t+\beta(t)$

The angle, $\zeta^{\prime}(t)$, can be computed from

$$
\begin{aligned}
\zeta^{\prime}(t) & =\sin ^{-1}[r \sin [\zeta(t)]](\operatorname{see}(87)) \\
& =\sin ^{-1}\left[r \sin \left\{k_{\alpha^{\prime}} t+\beta(t)\right\}\right]
\end{aligned}
$$

Next, we compute the derivative of the zenith angle, $\frac{d \zeta(t)}{d t}$, as follows:

$\frac{d \zeta(t)}{d t}=k_{\alpha^{\prime}}+\frac{d\left\{\tan ^{-1}\left[\frac{R_{e} \sin \left(k_{\alpha^{\prime}} t\right)}{R_{S^{-}-R_{e} \cos \left(k_{\alpha^{\prime}} t\right)}^{u(t)}}\right] \equiv \frac{\underbrace{\frac{f(t)}{R_{S}-R_{e} \cos \left(k_{\alpha^{\prime}} t\right)}}_{e^{\prime} \sin \left(k_{\alpha^{\prime}} t\right)}}{g(t)}\right\}}{d t}$ 


$$
=k_{\alpha^{\prime}}+\frac{1}{1+[\tan \beta(t)]^{2}} \frac{\frac{d f(t)}{d t} g(t)-f(t) \frac{d g(t)}{d t}}{g^{2}(t)}
$$

Where

$$
\begin{aligned}
& \frac{d f(t)}{d t}=R_{e} k_{\alpha^{\prime}} \cos \left(k_{\alpha^{\prime}} t\right) \\
& \frac{d g(t)}{d t}=R_{e} k_{\alpha^{\prime}} \sin \left(k_{\alpha^{\prime}} t\right)
\end{aligned}
$$

Substituting (109) and (110) into (108) yields

$$
\begin{aligned}
\frac{d \zeta(t)}{d t} & =k_{\alpha^{\prime}}+\frac{R_{e} k_{\alpha^{\prime}}}{1+[\tan \beta(t)]^{2}} \frac{\cos \left(k_{\alpha^{\prime}} t\right) g(t)-f(t) \sin \left(k_{\alpha^{\prime}} t\right)}{g^{2}(t)} \\
& =k_{\alpha^{\prime}}+\frac{R_{S} R_{e} k_{\alpha^{\prime}} \cos \left(k_{\alpha^{\prime}} t\right)-R_{e}{ }^{2} k_{\alpha^{\prime}}}{R_{S}{ }^{2}-2 R_{S} R_{e} \cos \left(k_{\alpha^{\prime}} t\right)+R_{e}{ }^{2}} \\
& =k_{\alpha^{\prime}}\left[1+\frac{R_{S} R_{e} \cos \left(k_{\alpha^{\prime}} t\right)-R_{e}{ }^{2}}{R_{S}{ }^{2}-2 R_{S} R_{e} \cos \left(k_{\alpha^{\prime}} t\right)+R_{e}{ }^{2}}\right] \\
& =\frac{k_{\alpha^{\prime}} R_{S}\left[R_{S}-R_{e} \cos \left(k_{\alpha^{\prime}} t\right)\right]}{R_{S}{ }^{2}-2 R_{S} R_{e} \cos \left(k_{\alpha^{\prime}} t\right)+R_{e}{ }^{2}}
\end{aligned}
$$

Next, let us find the derivative of $\frac{d\left\{m_{i}[\zeta(t)]\right\}}{d t}$ as follows

$$
\begin{aligned}
& \frac{d m_{i}(t)}{d t}=\frac{d\left[\sec \left[\frac{\sin ^{-1}\{r \sin [\zeta(t)]\}}{u(t)=\zeta^{\prime}(t)}\right]\right]}{d t} \\
& =\sec \left[\zeta^{\prime}(t)\right] \tan \left[\zeta^{\prime}(t)\right] \frac{d u(t)}{d t} \\
& =\frac{\sec [u(t)] \tan [u(t)] d\left[\sin ^{-1}[\underbrace{r \sin [\zeta(t)]}_{\varrho(t)}]\right]}{d t} \\
& =\frac{\sec [u(t)] \tan [u(t)]}{\sqrt{1-\varrho^{2}(t)}} \frac{d \varrho(t)}{d t} \\
& =r \frac{\sec \left[\zeta^{\prime}(t)\right] \tan \left[\zeta^{\prime}(t)\right] \cos [\zeta(t)]}{\sqrt{1-\sin ^{2}\left[\zeta^{\prime}(t)\right]}} \frac{d \zeta(t)}{d t} \\
& =r \frac{\sin \left[\zeta^{\prime}(t)\right] \cos [\zeta(t)]}{\cos ^{3}\left[\zeta^{\prime}(t)\right]} \frac{d \zeta(t)}{d t} \\
& =m_{i}^{\prime z}(t) \frac{d \zeta(t)}{d t}
\end{aligned}
$$

Where $\frac{d[\zeta(t)]}{d t}$ is already given previously by (110).

If we wanted to include the gradient component in the SID, $D_{i}^{s y}(t)$ (or $\left.\mathcal{D}_{i}^{a s}(t)\right)$ then we get

$D_{i}^{a s}(t)=D_{i}^{s y}(t)+D_{i}^{a z}(t)$

$$
=m_{i}(t) D_{i}^{z}(t)+m_{i}^{a z}(\zeta) D_{i}^{a z, z c}(t)
$$

$$
\begin{aligned}
\mathcal{D}_{i}^{a s}(t) & =\mathcal{D}_{i}^{s y}(t)+\mathcal{D}_{i}^{a z}(t) \\
& =m_{i}(t) D_{i}^{z}(t)+m_{i}^{a z}(\theta) D_{i}^{a z, z c}(t)
\end{aligned}
$$

Differentiating with respect to time we get the total Doppler frequency, $f_{i}^{D, a s}$ (or $f_{i}^{\mathcal{D}, a s}$ ) (see Figs. 27, 28 and Figs. 31, 32) as a result of the total ionospheric delay [11]

$$
\begin{aligned}
f_{i}^{D, a s} & =f_{i}^{D, s y}+f_{i}^{D, a z} \\
& =-\frac{\frac{d}{d t} D_{i}^{s y}(t)}{\lambda}-\frac{\frac{d}{d t} D_{i}^{a z}(t)}{\lambda} \\
& =-\frac{\frac{d m_{i}(t) D_{i}^{z}(t)}{d t}}{\lambda}-\frac{\frac{d m_{i}^{a z}(\zeta) D_{i}^{a z, z c}(t)}{d t}}{\lambda} \\
f_{i}^{\mathcal{D}, a s} & =f_{i}^{\mathcal{D}, s y}+f_{i}^{\mathcal{D}, a z} \\
& =-\frac{\mathcal{D}_{i}^{s y}(t)}{\lambda}-\frac{\frac{d}{d t} \mathcal{D}_{i}^{a z}(t)}{\lambda} \\
& =-\frac{\frac{d m_{i}(t) D_{i}^{z}(t)}{d t}}{\lambda}-\frac{\frac{d m_{i}^{a z}(\theta) D_{i}^{a z, z c}(t)}{d t}}{\lambda}
\end{aligned}
$$

The symmetric ionospheric Doppler frequency, $f_{i}^{D, s y}$ (or $\left.f_{i}^{\mathcal{D}, s y}\right)$, is simply the derivative of the ionospheric slant delay, $D_{i}^{s y}(t)$ (or $\left.\mathcal{D}_{i}^{s y}(t)\right)$, with respect to time, $t$, or

$$
\begin{aligned}
f_{i}^{D, s y} & =-\frac{\frac{d D_{i}^{s y}(t)}{d t}}{\lambda} \\
& =-\frac{\frac{d m_{i}(t) D_{i}^{Z}(t)}{d t}}{\lambda} \\
& =-\frac{m_{i}(t) \frac{d D_{i}^{Z}(t)}{d t}+D_{i}^{Z}(t) \frac{d m_{i}(t)}{d t}}{\lambda} \\
f_{i}^{\mathcal{D}, s y} & =-\frac{\frac{d D_{i}^{s y}(t)}{d t}}{\lambda} \\
& =-\frac{\frac{d m_{i}(t) D_{i}^{Z}(t)}{d t}}{\lambda} \\
& =-\frac{m_{i}(t) \frac{d D_{i}^{Z}(t)}{d t}+D_{i}^{Z}(t) \frac{d m_{i}(t)}{d t}}{\lambda}
\end{aligned}
$$

There are two components to the symmetric Doppler frequency, $f_{i}^{D}$ (or $f_{i}^{\mathcal{D}}$ ): (1), as a result of the change in ionospheric zenith delay, $D_{i}^{z}(t)$ (see (65)), and (2) the other as a result of the change in the obliquity factor $m_{i}\left(\zeta^{k}\right)$ (or $\left.m_{i}\left(\theta^{k}\right)\right)($ see $(85)$ or $(86))$.

First, we compute the first component of the Doppler frequency as a result of the change in ionospheric zenith delay, $D_{i}^{z}(t)$; i.e.,

$\frac{d D_{i}^{Z}(t)}{d t}=-\frac{a \frac{d T E C(x, t)}{d t}}{c f}$

Second, we compute the second component of the Doppler frequency as a result of the change in the obliquity factor 


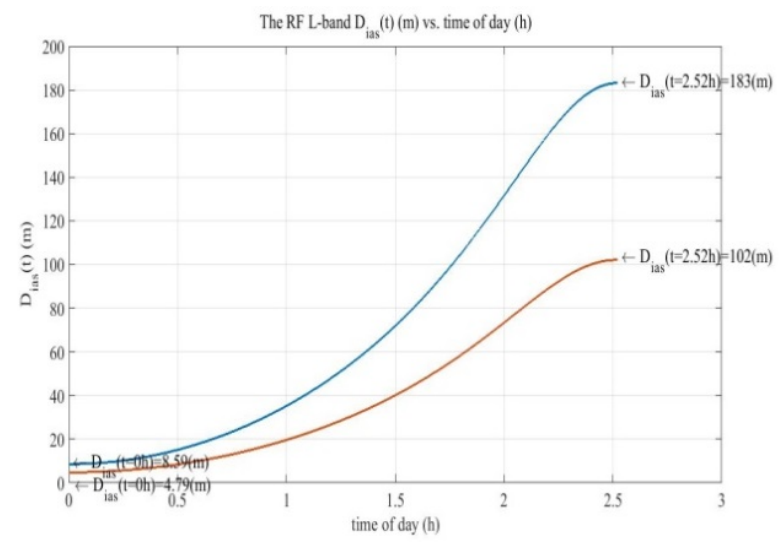

Fig. 29. Total ionospheric delay, $D_{i}^{a s}(t)(\mathrm{m})$, as a function of time of day (h) using the second profile.

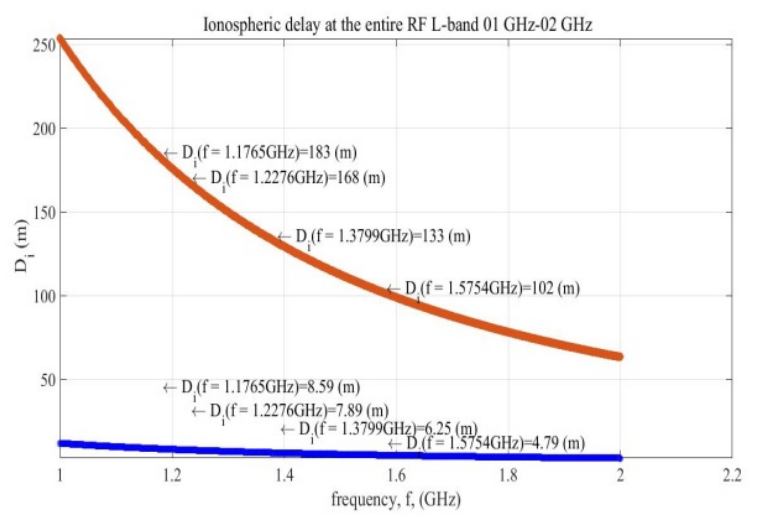

Fig. 30. Total ionospheric delay, $D_{i}^{a s}(t)(\mathrm{m})$, as a function of frequency $(\mathrm{GHz})$ using the second profile.

$m_{i}\left(\zeta^{k}\right)\left(\right.$ or $\left.m_{i}\left(\theta^{k}\right)\right)$; i.e.,

$\frac{d m_{i}(t)}{d t}=-\frac{m_{i}^{\prime z}(t)}{\lambda} \frac{d \zeta(t)}{d t}($ see $(111))$

$\frac{d m_{i}(t)}{d t}=-\frac{m_{i}^{\prime Z}(t)}{\lambda} \frac{d \theta(t)}{d t}$

Where

$$
m_{i}^{\prime z}(t)=\frac{-r^{2} \cos \left[\theta^{\prime}(t)\right] \sin [\theta(t)]}{\sin ^{3}\left[\theta^{\prime}(t)\right]}
$$

Finally substituting (118) and (119) or (120) into (117) we obtain Doppler frequency, $f_{i}^{D}$ (or $f_{i}^{\mathcal{D}}$ ): (1), as a result of the change in ionospheric zenith delay, $D_{i}^{Z}(t)$ (see (65)), and (2) the other as a result of the change in the obliquity factor $m_{i}\left(\zeta^{k}\right)$ (or $\left.m_{i}\left(\theta^{k}\right)\right)$ (see $(85)$ or $\left.(86)\right)$; i.e.,

$$
\begin{aligned}
& f_{i}^{D, s y}(t)=-\frac{a \frac{d T E C(x, t)}{d t}}{c f}-\frac{D_{i}^{z}(t) \gamma(t)}{\lambda} \frac{d \zeta(t)}{d t} \\
& f_{i}^{\mathcal{D}, s y}(t)=-\frac{a \frac{d T E C(x, t)}{d t}}{c f}-\frac{D_{i}^{Z}(t) m_{i}^{\prime z}(t)}{\lambda} \frac{d \theta(t)}{d t}
\end{aligned}
$$

Since, the (or symmetric) Doppler component $f_{i}^{D, s y}$ (or $\left.f_{i}^{\mathcal{D}, s y}\right)$ is already computed in (122) or (123); the azimuth

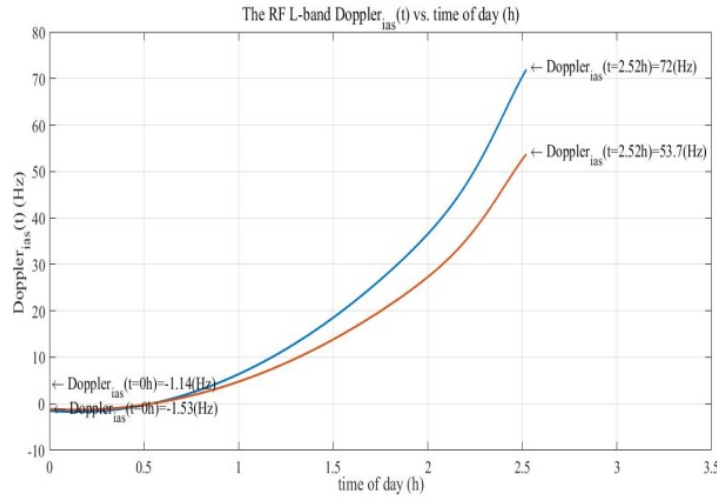

Fig. 31. Total ionospheric Doppler, $f_{i}^{D, a s}(t)(\mathrm{Hz})$, as a function of time of day (h) using the second profile.

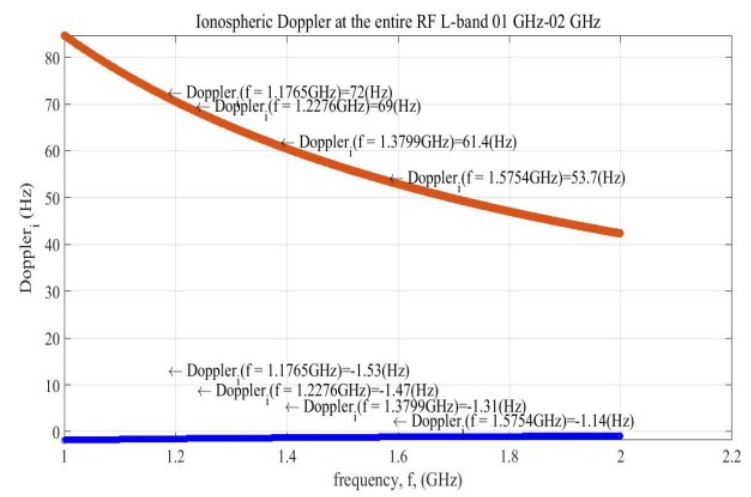

Fig. 32. Total ionospheric Doppler, $f_{i}^{D, a s}(t)(\mathrm{Hz})$, as a function of frequency $(\mathrm{GHz})$ using the second profile.

Doppler component from $f_{i}^{D, a z}$ (or $f_{i}^{\mathcal{D}, a z}$ ) can be computed from

$$
\begin{aligned}
f_{i}^{D, a z}(t) & =-\frac{\frac{d}{d t} D_{i}^{a z}[\zeta(t), t] \equiv m_{i}^{a z}(\zeta) D_{i}^{a z, z c}(t)}{\lambda} \\
& =-\frac{\frac{d}{d t} D_{i}^{a z}[\zeta(t), t] \equiv \sin \left[\zeta^{\prime}(t)\right] \tan \left[\zeta^{\prime}(t)\right] D_{i}^{a z, z c}(t)}{\lambda} \\
& =-\frac{\frac{d\left\{\sin \left[\zeta^{\prime}(t)\right] \tan \left[\zeta^{\prime}(t)\right]\right\}}{d t} D_{i}^{a z, z c}(t)+m_{i}^{a z}(\zeta) \frac{d D_{i}^{a z, z c}}{d t}}{\lambda} \\
& =-\frac{\frac{1+\sec ^{2}\left[\zeta^{\prime}(t)\right] d \zeta^{\prime}(t)}{\csc \left[\zeta^{\prime}(t)\right]} D_{i}^{a z, z c}(t)+m_{i}^{a z}(\zeta) \frac{d D_{i}^{a z, z c}(t)}{d t}}{\lambda}
\end{aligned}
$$

$$
\begin{aligned}
f_{i}^{\mathcal{D}, a z}(t) & =-\frac{\frac{d}{d t} \mathcal{D}_{i}^{a z}[\theta(t), t] \equiv d m_{i}^{a z}(\theta) D_{i}^{a z, z c}(t)}{\lambda} \\
& =-\frac{\frac{d}{d t} \mathcal{D}_{i}^{a z}[\theta(t), t] \equiv \cos \left[\theta^{\prime}(t)\right] \cot \left[\theta^{\prime}(t)\right] D_{i}^{a z, z c}(t)}{\lambda} \\
& =-\frac{\frac{d\left\{\cos \left[\theta^{\prime}(t)\right] \cot \left[\theta^{\prime}(t)\right]\right\}}{d t} D_{i}^{a z, z c}(t)+m_{i}^{a z}(\theta) \frac{d D_{i}^{a z, z c}(t)}{d t}}{\lambda}
\end{aligned}
$$$$
=\frac{\frac{\frac{1+\csc ^{2}\left[\theta^{\prime}(t)\right]}{\operatorname{scs}\left[\theta^{\prime}(t)\right]} d \zeta^{\prime}(t)}{d t} D_{i}^{a z, z c}(t)-m_{i}^{a z}(\theta) \frac{d D_{i}^{a z, z c}(t)}{d t}}{\lambda}
$$ 
Since, $\zeta^{\prime}(t)$ is given by (106); hence,

$\frac{d \zeta^{\prime}(t)}{d t}=\frac{r \cos [\zeta(t)]}{\cos \left[\zeta^{\prime}(t)\right]} \frac{d[\zeta(t)]}{d t}$

Similarly,

$\theta^{\prime}(t)=\cos ^{-1}\{r \cos [\theta(t)]\}$

$\frac{d \theta^{\prime}(t)}{d t}=\frac{r \sin [\theta(t)]}{\sin \left[\theta^{\prime}(t)\right]} \frac{d[\theta(t)]}{d t}$

However, since $\theta(t)=90^{\circ}-\zeta(t)$ then; hence,

$$
\frac{d \theta(t)}{d t}=\frac{-d \zeta(t)}{d t}
$$

Finally, substituting (126), (128)/(129), into (124)/(125) we obtain

$$
\begin{aligned}
& f_{i}^{D, a z}(t)=-\frac{m_{i}^{\prime a z}(t) D_{i}^{a z, z c} \frac{d \zeta(t)}{d t}+m_{i}^{a z}(\zeta) \frac{d D_{i}^{a z, z c}}{d t}}{\lambda} \\
& f_{i}^{\mathcal{D}, a z}(t)=-\frac{m_{i}^{\prime a z}(t) \mathcal{D}_{i}^{a z, z c} \frac{d \theta(t)}{d t}+m_{i}^{a z}(\theta) \frac{d D_{i}^{a z, z c}}{d t}}{\lambda}
\end{aligned}
$$

Where

$$
\begin{aligned}
& m_{i}^{\prime a z}(t)=\frac{\sin \left[\zeta^{\prime}(t)\right]\left[1+\sec ^{2}\left[\zeta^{\prime}(t)\right]\right] r \cos [\zeta(t)]}{\cos \left[\zeta^{\prime}(t)\right]} \\
& m_{i}^{\prime a z}(t)=\frac{\cos \left[\theta^{\prime}(t)\right]\left[1+\csc ^{2}\left[\theta^{\prime}(t)\right]\right] r \sin [\theta(t)]}{\sin \left[\theta^{\prime}(t)\right]}
\end{aligned}
$$

Next, let us compute $\frac{d D_{i}^{a z, z c}(t)}{d t}$ by differentiating (78); therefore, first we compute

$$
\begin{aligned}
& \frac{d \frac{c_{1}}{\sqrt{1+\varsigma^{2}}}}{d t}=\frac{\frac{d c_{1}}{d t} \sqrt{1+\varsigma^{2}}-c_{1} \frac{d \sqrt{1+\left(\frac{c_{1}}{c_{2}}\right)^{2}}}{d t}}{1+\varsigma^{2}} ; \varsigma=\frac{c_{1}}{c_{2}} \\
& =\frac{\frac{d c_{1}}{d t} \sqrt{1+\varsigma^{2}}-0.5 c_{1} \frac{\frac{d\left(\frac{c_{1}}{c_{2}}\right)^{2}}{d t}}{\sqrt{1+\varsigma^{2}}}}{1+\varsigma^{2}} \\
& =\frac{\frac{d c_{1}}{d t}\left[1+\varsigma^{2}\right]-c_{1} \frac{c_{1}\left[\frac{d c_{1}}{d t} c_{2}-c_{1} \frac{d c_{2}}{d t}\right]}{c_{2} c_{2}^{2}}}{\left(1+\varsigma^{2}\right)^{\frac{3}{2}}} \\
& =\frac{\frac{d c_{1}}{d t}\left(1+\varsigma^{2}\right)-\frac{d c_{1}}{d t} \varsigma^{2}+\varsigma^{3} \frac{d c_{2}}{d t}}{\left(1+\varsigma^{2}\right)^{\frac{3}{2}}} \\
& =\frac{\frac{d c_{1}}{d t} \sqrt{1+\varsigma^{2}}-0.5 c_{1} \frac{\frac{d\left(\frac{c_{1}}{c_{2}}\right)^{2}}{d t}}{\sqrt{1+\varsigma^{2}}}}{1+\varsigma^{2}} \\
& =\left(\frac{d c_{1}}{d t}+\varsigma^{3} \frac{d c_{2}}{d t}\right)\left[1+\varsigma^{2}\right]^{-\frac{3}{2}} \rightarrow
\end{aligned}
$$

$\frac{d \frac{c_{2}}{\sqrt{1+\varsigma^{2}}}}{d t}=\left(\frac{d c_{2}}{d t}+\varsigma^{3} \frac{d c_{1}}{d t}\right)\left[1+\varsigma^{2}\right]^{-\frac{3}{2}}$

Hence,

$\frac{d D_{i}^{a z, z c}(t)}{d t}=-\frac{2 a\left(\frac{d c_{1}}{d t}+\varsigma^{3} \frac{d c_{2}}{d t}\right)}{f_{k}^{2}\left(1+\varsigma^{2}\right)^{\frac{3}{2}}}$, if $c_{1} \leq c_{2}$

$\frac{d D_{i}^{a z, z c}(t)}{d t}=-\frac{2 a\left(\frac{d c_{2}}{d t}+\varsigma^{-3} \frac{d c_{1}}{d t}\right)}{f_{k}^{2}\left(1+\varsigma^{-2}\right)^{\frac{3}{2}}}$, if $c_{2} \geq c_{1}$

Finally, substituting (122)/(123), (120)/(131) into (114)/(115) we obtain the final Doppler expression due to total ionospheric delay [11]

$f_{i}^{D, a s}(t)=-\left[\frac{a m_{i}(t) \frac{d T E C(x, t)}{d t}}{c f}+\frac{D_{i}^{\prime m}(t) \frac{d \zeta(t)}{d t}+\frac{m_{i}^{a z}(\zeta) d D_{i}^{a z, z c}(t)}{d t}}{\lambda}\right]$

$f_{i}^{\mathcal{D}, a s}(t)=-\left[\frac{a m_{i}(t) \frac{d T E C(x, t)}{d t}}{c f}+\frac{\mathcal{D}_{i}^{\prime m}(t) \frac{d \theta(t)}{d t}+\frac{m_{i}^{a z}(\theta) d D_{i}^{a z, z c}(t)}{d t}}{\lambda}\right]$

Where

$$
\begin{aligned}
& D_{i}^{\prime m}(t)=m_{i}^{\prime z}(t) D_{i}^{z}(t)+m_{i}^{\prime a z}(t) D_{i}^{a z, z c}(t) \\
& \mathcal{D}_{i}^{\prime m}(t)=m_{i}^{\prime z}(t) D_{i}^{z}(t)+m_{i}^{\prime a z}(t) D_{i}^{a z, z c}(t)
\end{aligned}
$$

Special cases: There are four special cases that we should take into consideration.

(1) Static component. If we assume that $\frac{d \zeta(t)}{d t}=\frac{d \theta(t)}{d t}=0$ then $A(t)=B(t)=0$.

$$
\begin{aligned}
& f_{i}^{D, a s}(t)=-\frac{a m_{i}(t) \frac{d T E C(x, t)}{d t}}{c f}-\frac{m_{i}^{a z}(\zeta)}{\lambda} \frac{d D_{i}^{a z, z c}(t)}{d t} \\
& f_{i}^{\mathcal{D}, a s}(t)=-\frac{a m_{i}(t) \frac{d T E C(x, t)}{d t}}{c f}-\frac{m_{i}^{a z}(\theta)}{\lambda} \frac{d D_{i}^{a z, z c}(t)}{d t}
\end{aligned}
$$

If $m_{i}(t)=m_{i}(t)=1$ then the static component becomes the same as the zenith component; i.e., zenith component is a subcomponent of the static component.

(a) Zenith component with no gradient component. On the other hand, another effect known as the phase advance $\Delta \phi_{k}$ affects the carrier frequency of the transmitted signal, $f_{k}$. In this case the signal is affected by the following factor in cycles (or in radians) [81]

$$
\begin{aligned}
\Delta \phi_{k c} & =\frac{a \cdot T E C}{c f_{k}}=f_{k} \frac{D_{i}^{z}}{c} \text { (in cycles) } \\
\Delta \phi_{k} & =2 \pi \Delta \phi_{k c}=\omega_{k} \frac{D_{i}^{z}}{c} \text { (in radians) }
\end{aligned}
$$

where (1) $D_{i}^{Z}$ is the ionospheric zenith delay in [m]; (2) $c$ is the SOL in vacuum [2] [m/s].

Therefore, if one was to assume the ionosphere [2] to be a 
Journal of Geolocation, Geo-information, and Geo-intelligence

layer between the satellite and the user, the TEC encountered by the signal directly depends on the satellite elevation and change as the satellite is moving. This change induces a change in the phase advance observed and as such a frequency shift known as the Doppler frequency induced by the ionosphere [2] given by [81], [82]

$$
\begin{aligned}
\Delta \phi_{k}= & \frac{d \Delta \phi_{k}}{d t} \\
& =f_{i}^{D}(t) \\
& =-\frac{a}{c f_{k}} \frac{d T E C(t)}{d t} \\
& =\left[\frac{1.344536593729647 \times 10^{-7}}{f_{k}} \cdot \frac{\text { Hz.cycles }}{\text { TECN }}\right] \frac{d T E C}{d t} \\
& =-\frac{f_{k}}{c} \frac{d D_{i}^{Z}(t)}{d t}
\end{aligned}
$$

Where

$$
\begin{aligned}
a & =1.344536593729647 \times 10^{-7} \mathrm{els}^{-1} \cdot \mathrm{m}^{2} \cdot \mathrm{s}^{-1} \cdot c \\
& =\frac{1.344536593729647}{10^{7}} \cdot \frac{\mathrm{m}^{2}}{\mathrm{els} \times s} \times 299,792,458 \frac{\mathrm{m}}{\mathrm{s}} \\
& =40.308193030515824 \mathrm{els}^{-1} \cdot \mathrm{m}^{3} \cdot \mathrm{s}^{-2}
\end{aligned}
$$

where TEC is the integral of electron density along the path of the radio signal from transmitter to receiver, where $f_{1,2}$ is evaluated in units [83] $\mathrm{Hz}, d T E C / d t$ is evaluated in units $\mathrm{TECN} / \mathrm{s}$, and where $\mathrm{TECN}=1 \mathrm{el} \cdot \mathrm{m}^{-2}$ [81].

(2) Slant (symmetric) component with and no change in TEC and no gradient component. With dual frequency measurements, we can compute the ionospheric delay [11] and numerically differentiate to produce an additional correction term to the predicted model Doppler. However, without this information, we can assume that the path delay is a function of the zenith delay and the obliquity factor (itself a function of the satellite elevation). Differentiating the path slant delay, $\mathcal{D}_{i}^{s y}(\theta)$, with respect to time, we find that

$$
\begin{aligned}
& \frac{d \mathcal{D}_{i}^{s y}(t, \theta)}{d t}=-D_{i}^{Z}(t) m_{i}^{\prime z}(t) \frac{d \theta(t)}{d t} \rightarrow \\
& f_{i}^{\mathcal{D}}(t)=-\frac{1}{\lambda_{1}} \frac{d \mathcal{D}_{i}^{s y}(\theta)}{d t}=\frac{D_{i}^{Z}(t)}{\lambda_{1}} m_{i}^{\prime Z}(t) \frac{d \theta(t)}{d t}(\operatorname{see}(121))
\end{aligned}
$$

where $\theta$ is the elevation angle of the lowest layer, $\mathcal{D}_{i}^{Z}$ is the zenith delay in meters. The Doppler shift from the ionosphere [2] is the negative of the time rate of change of the path delay over the wavelength [84].

The negative sign is due to the phase advance being opposite in sign from the path delay. The new predicted frequency is thus

$$
\hat{f}_{i}^{D}=f^{\text {model }}+f_{i}^{D}
$$

Leveraging dual-frequency code-based pseudoranges from the SATRACK data, we calculated an approximate zenith delay for each space vehicle (SV), which varied between $1.5 \mathrm{~m}$ and $3 \mathrm{~m}$. The total effect of $f_{i}{ }^{D}$ is small (thousandths of $\mathrm{Hz}$ ) but can correct some of the trends observed in the residual phase measurements [84].

The same result in (149) can be obtained from (139) if $\frac{d T E C(x, t)}{d t}=0$ and $D_{i}^{a z, z c}(t)=0$ then $\frac{d D_{i}^{a z, z c}(t)}{d t}=0$.

(3) Slant (symmetric) component with no gradient component.

If we assume $D_{i}^{a z, z c}=0$ then $\frac{d D_{i}^{a z, z c}}{d t}=0$ then from $(137) /(138)$ the following is obtained

$$
\begin{aligned}
& f_{i}^{D, s y}(t)=-\frac{a m_{i}(t) \frac{d T E C(x, t)}{d t}}{c f}-\frac{D_{i}^{z}(t) m_{i}^{\prime z}(t)}{\lambda} \frac{d \zeta(t)}{d t} \\
& f_{i}^{\mathcal{D}, s y}(t)=-\frac{a m_{i}(t) \frac{d T E C(x, t)}{d t}}{c f}-\frac{D_{i}^{z}(t) m_{i}^{\prime z}(t)}{\lambda} \frac{d \theta(t)}{d t}
\end{aligned}
$$

This is the slant (symmetric) Doppler component with no gradient component same as (122)/(123).

(4) Slant azimuth Doppler component. If we assume $\frac{\operatorname{dTEC}(x, t)}{d t}=0$ then from $(138) /(139)$ the following is obtained

$f_{i}^{D, a s}=-\frac{D_{i}^{\prime m}(t) \frac{d \zeta(t)}{d t}+\frac{m_{i}^{a z}(\zeta) d D_{i}^{a z, z c}(t)}{d t}}{\lambda}$

$$
f_{i}^{\mathcal{D}, a s}=-\frac{\mathcal{D}_{i}^{\prime m}(t) \frac{d \theta(t)}{d t}+m_{i}^{a z}(\theta) \frac{d \mathcal{D}_{i}^{a z, z c}(t)}{d t}}{\lambda}
$$

where $D_{i}^{\prime m}(t)$ and $D_{i}^{\prime m}(t)$ are given by (140) and (141).

This is the slant azimuth Doppler component; i.e., gradient and derivative of the gradient only component with no change in TEC, neither zenith nor elevation component. Furthermore, slant azimuth Doppler component can be decomposed into two subcomponents:

(a) gradient only with no change in TEC if $\frac{d D_{i}^{a z, z c}(t)}{d t}=0$ or $D_{i}^{a z, z c}=$ const.

$f_{i}^{D, a s}(t)=-\frac{D_{i}^{z}(t) \gamma(t) \frac{d \zeta(t)}{d t}}{\lambda}-\frac{m_{i}^{\prime a z}(t) D_{i}^{a z, z c}(t)}{\lambda}$

$f_{i}^{\mathcal{D}, a s}(t)=-\frac{D_{i}^{Z}(t) \delta(t) \frac{d \theta(t)}{d t}}{\lambda}-\frac{m_{i}^{\prime a z}(t) D_{i}^{a z, z c}(t)}{\lambda}$

and (b) derivative of the gradient only with no change in TEC 
if $\frac{d \zeta(t)}{d t}=\frac{d \theta(t)}{d t}=0$ then $m_{i}^{\prime a z}(t)=m_{i}^{\prime a z}(t)=0$ hence

$$
\begin{aligned}
& f_{i}^{D, a s}(t)=-\frac{m_{i}^{a z}(\zeta)}{\lambda} \frac{d D_{i}^{a z, z c}(t)}{d t} \\
& f_{i}^{\mathcal{D}, a s}(t)=-\frac{m_{i}^{a z}(\theta)}{\lambda} \frac{d D_{i}^{a z, z c}(t)}{d t}
\end{aligned}
$$

This concludes the description of the ionospheric Doppler frequency calculations.

\subsection{Description of the amplitude and phase scintillation}

Amplitude scintillation is a measure of the signal amplitude degradation due to scintillation and is measured by an index known as $S_{4}$.

The $S_{4}$ amplitude scintillation index [85] is computed as the standard deviation of normalized signal intensity [86] (or irradiance, $I$ ) (expressed in linear units [83], not $\mathrm{dB}$ ) or power $P$.

$$
S_{4}^{2}=\frac{\left\langle I^{2}\right\rangle-\langle I\rangle^{2}}{\langle I\rangle^{2}}=\frac{\frac{\left\langle P^{2}\right\rangle}{A^{2}}-\frac{\langle P\rangle^{2}}{A^{2}}}{\frac{\langle P\rangle^{2}}{A^{2}}}=\frac{\left\langle P^{2}\right\rangle-\langle P\rangle^{2}}{\langle P\rangle^{2}}
$$

If the signal is measured in power units (or Wats [22]) then $Y$ : Power $E[Y]=\langle P\rangle ; \sigma_{[Y]}=S_{4}\langle P\rangle$ else if the signal is measured in amplitude units then $X$ : Amplitude $E[X]=\langle A\rangle=$ $\sqrt{2\langle P\rangle} ; \sigma_{[X]}=\sqrt{2 S_{4}\langle P\rangle}=\sqrt{S_{4}}\langle A\rangle=\sqrt{2 \sigma_{[Y]}}$.

Here the angle brackets $\langle a\rangle \equiv E\{a\}$ formally denote an ensemble average of $a$ or $E\{a\}$ but in practice it indicates spatial or temporal averages.

One school of thought believes that the $S_{4}$ index used as a sole measure of scintillation does neither parametrerize phase fluctuation index nor provides detailed characterization of fade depth or duration. According to this school of thought, once $S_{4}$ reaches the neighborhood of unity (focusing on saturation regime), scintillation characteristics may vary even as $S_{4}$ remains relatively constant. Nevertheless, $S_{4}$ remains a worthwhile means of characterizing scintillation due to several factors: (1) large, long term activities of $S_{4}$ measurements exist and morphological studies use these archives the natural scintillation environment (Aarons, 1993 [87]); (2) a good number of scintillation detecting systems are largely based on $S_{4}$ measurements (Groves et al, 1997, [88]; Thomas et al., 2001, [89]); (3) additionally scintillation at L-band (1-2 GHz) or higher frequencies often does not approach saturation except in limited circumstances (Fremouw, et al, 1980 [90]; Yeh and Liu, 1982 [91]); (4) finally, empirical relationships between $S_{4}$ and properties like signal fade statistics allow historical or climatological $S_{4}$ values to be used in system design [85]. As we are going to see from the derivations and simulation analyses, the conclusions provided by this school of thought are erroneous.

Another school of thought, as we shall see in the further development of this section, believes that there exists a welldefined relationship between the phase fluctuations and the amplitude scintillation index, $S_{4}$. Therefore, we are first going to produce an understanding about the phase fluctuations or phase scintillation index or phase variance due to scintillation and then derive the amplitude scintillation index, $S_{4}$ [80], [92][94].

In Jandieri et al. 2009 [80] phase fluctuations are suggested as being responsible for fluctuations of the angle-of-arrival (AOA). AOA measurements have been performed by interferometers; where as a part of radar propagation effects program at the Millstone Hill radar facility [1], AOAs has been measured with a single mono-pulse tracking system. According to Jandieri et al. 2009 [80] the study of AOA could provide useful information about the structure of the ionospheric irregularities.

Let as consider a simplified model of the magnetized plasma in order to visualize the influence of medium absorption and anisotropy on the AOA of scattered waves [80].

Let us assume a 3-layer approximation between a Satellite Transmitter and a Receiver with respective thickness, $h_{i}, i=$ $\{1,2,3\}$ as shown in Fig. 12; where the middle, scattered layer contains random magnetized plasma and irradiated highfrequency electromagnetic waves.

The LOS distance between the Satellite Transmitter and a Receiver is given by

$$
H_{0}=\sum_{i=1}^{3} h_{i}
$$

Suppose a magnetic field with constant flux density vector, $\mathbf{B}_{0}$, and magnitude of $B_{0}$ and angle of $\zeta_{0}$ from the $\mathrm{z}$ axis is given by

$$
\mathbf{B}_{0}=B_{0} e^{j \zeta_{0}}
$$

Suppose that the wavenumber vector, $\mathbf{k}$, of the signal in vacuum is given by

$$
\mathbf{k}=k_{0} e^{j\left(\zeta^{\prime} \equiv \beta\right)}
$$

where the wavenumber magnitude of the signal in vacuum, whose radiant frequency is $\omega$, is given by

$$
k_{0}=\omega / c
$$




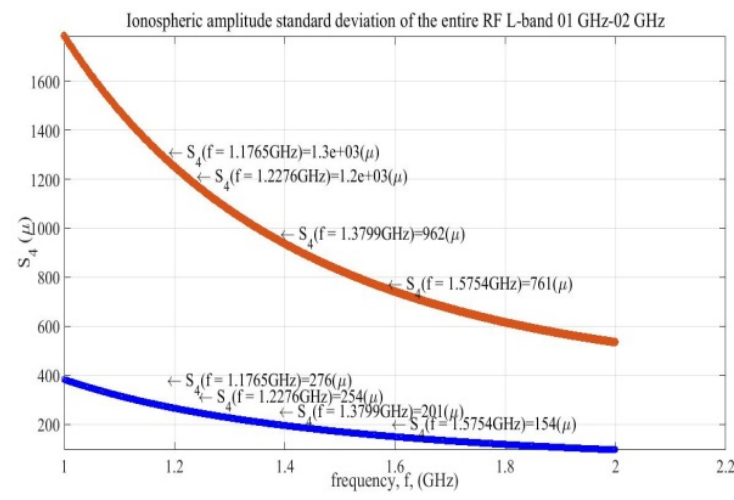

Fig. 33. Total ionospheric amplitude standard derivation, $S_{4}(\mu)$, as a function of frequency $(\mathrm{GHz})$ using the first profile.

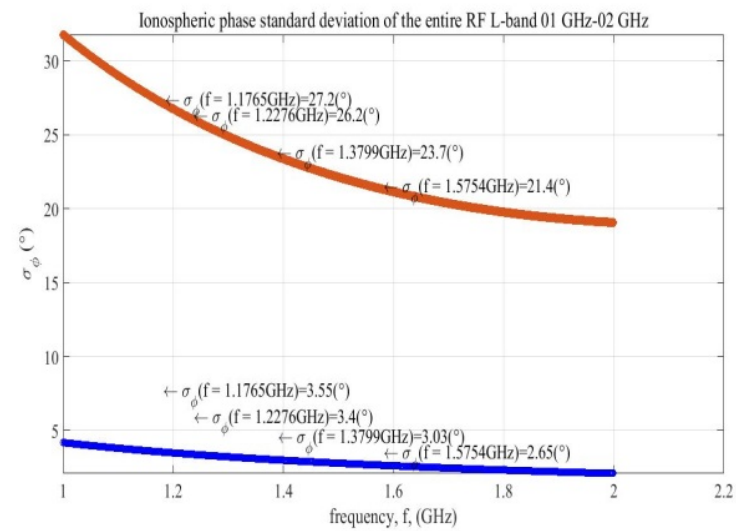

Fig. 34. Total ionospheric phase standard derivation, $\sigma_{\phi}\left({ }^{\circ}\right)$, as a function of frequency $(\mathrm{GHz})$ using the first profile.

Suppose that the plasma density in the layer, $N(\boldsymbol{r})$, is the sum of two terms: the constant term, $N_{0}$, and the random function of the spatial coordinates describing density fluctuations, $N_{1}(\boldsymbol{r})$, then $N(\boldsymbol{r})$ can be written as [80]

$$
N(\boldsymbol{r})=N_{0}+N_{1}(\boldsymbol{r})
$$

Assuming a Gaussian distribution of the random function of the spatial coordinates plasma density fluctuations, $N_{1}(\boldsymbol{r})$, its variance is given by $\left\langle N_{1}^{2}\right\rangle$; hence, the variance of the plasma density in the layer, $N(\boldsymbol{r})$, is denoted by $\sigma_{N}^{2}$ and it can be computed from

$$
\sigma_{N}^{2}=\left\langle N_{1}^{2}\right\rangle / N_{0}^{2}
$$

Let as assume that $v_{\text {eff }}$ is the effective collision frequency of electrons with other plasma particles; the plasma frequency can be computed from [80]

$$
\omega_{p}^{2}=\frac{e^{2} n_{e}}{\varepsilon_{0} m_{e}}=4 \pi^{2} f_{p}^{21}
$$

The angular gyro-frequency for the magnetic field, $\omega_{B}$, is

1 The plasma frequency, $f_{p}$, was introduced earlier in (40).

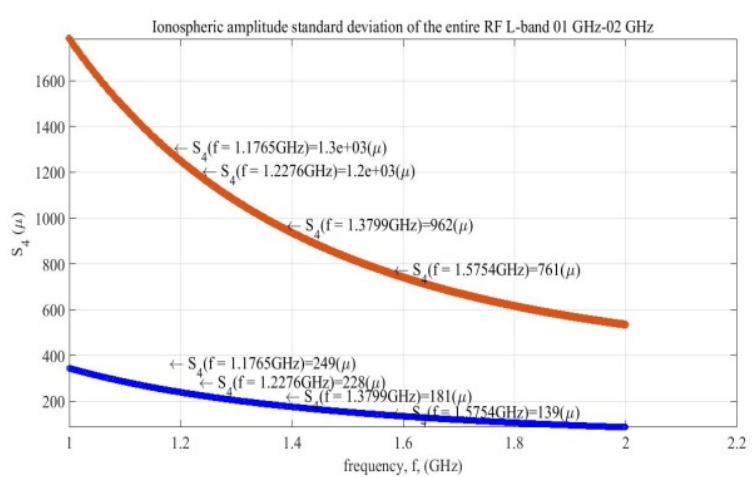

Fig. 35. Total ionospheric amplitude standard derivation, $S_{4}(\mu)$, as a function of frequency $(\mathrm{GHz})$ using the second profile.

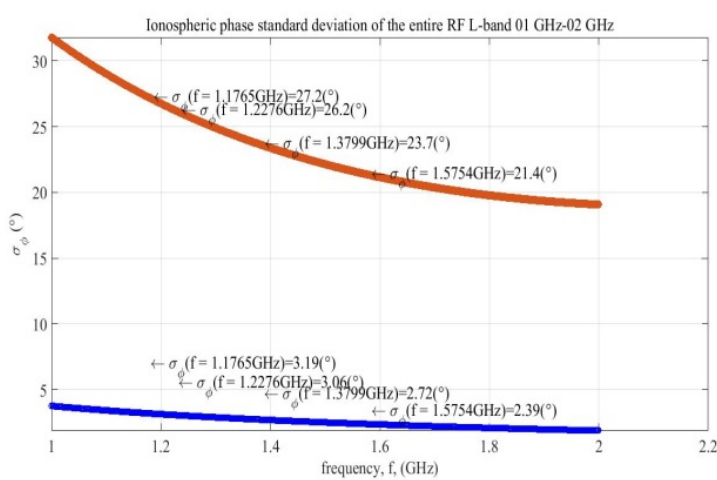

Fig. 36. Total ionospheric phase standard derivation, $\sigma_{\phi}\left({ }^{\circ}\right)$, as a function of frequency $(\mathrm{GHz})$ using the second profile.

defined as [80]

$$
\omega_{B}=\frac{e B_{0}}{m c}
$$

Suppose that the following conditions are fulfilled [80]

$\omega_{B} \gg \omega ; \omega_{B} \gg \omega_{p} ; \omega_{B} \gg v_{\mathrm{eff}}$

We define the following non dimensional plasma parameters $v$ and $s$ as follows

$$
\begin{aligned}
& v=\omega_{p}^{2} / \omega^{2} \\
& s=\mathrm{v}_{\mathrm{eff}} / \omega
\end{aligned}
$$

Anisotropic Gaussian correlation function of electron density fluctuations in F-region of ionospheric plasma, when prolate (or elongate along the polar diameter) irregularities produce an angle, $\gamma$, with respect to the external magnetic field.

Assuming that the irregularities are elongated along a direction of the geomagnetic field

$$
\alpha=k_{0}^{2}\left[\alpha_{0} \equiv \frac{1}{2 N_{0}} \frac{v \sin ^{2} \zeta_{0}}{\left(1-v \cos ^{2} \zeta_{0}\right)^{2}}\right]^{2}
$$

Then it can be easily shown that $\alpha>1$ [80].

\footnotetext{
2 Please do not confuse $\alpha$ with $\alpha^{\prime}$.
} 
Let $h_{\|}$and $h_{\perp}$ indicate the relative characteristic spatial scale sizes in the field aligned, $\|$, and field-perpendicular, $\perp$, directions; then $\chi$ is the anisotropic parameter (see Fig. 12) given by

$$
\begin{aligned}
& \chi=h_{\|} / h_{\perp} \\
& \bar{h}^{2}=\frac{h_{\|}^{2}}{\sin ^{2} \gamma+\chi^{2} \cos ^{2} \gamma}
\end{aligned}
$$

Next $a, b$, and $d$ are defined as non-dimensional spatial parameters normalizing on thickness of a plasma layer

$$
\begin{aligned}
& a=h_{\|} / h_{2} \\
& b=h_{1} / h_{2} \\
& d=h_{3} / h_{2}
\end{aligned}
$$

Next, we define the following non dimensional plasma parameters

$$
\begin{aligned}
& A=v \frac{b+d}{1+b+d} \sin \zeta_{0} \cdot \cos \zeta_{0}=v \gamma \\
& J_{0}=v \frac{b}{1+b+d} \sin \zeta_{0} \cdot \cos \zeta_{0}=v \delta
\end{aligned}
$$

Next, we define the following remaining parameters as follows:

$$
\begin{aligned}
& a_{1}=\frac{1+\frac{\left(1-\chi^{2}\right)^{2} \sin ^{2} \alpha \cdot \cos ^{2} \alpha}{\chi^{2}}}{\sin ^{2} \alpha+\chi \cos ^{2} \alpha} \\
& a_{2}=\frac{\sin ^{2} \alpha+\chi^{2} \cos ^{2} \alpha}{\chi^{2}} \\
& a_{3}=\frac{\left(1-\chi^{2}\right) \sin \alpha \cdot \cos \alpha}{\chi^{2}} \\
& G=a_{1}+a_{2} A^{2}-2 a_{3} A=a_{1}+a_{2} v^{2} \gamma^{2}-2 a_{3} v \gamma \\
& J=2 s J_{0}
\end{aligned}
$$

Assume that distance between two receivers normal to the direction of wave propagation in which phase correlation is measured is $x$ then the AOA and frequency fluctuations intensity for anisotropic Gaussian correlation function at $G_{0} \equiv$ $G(x=0)$ has the following form [80]

$$
\sigma_{\varphi}^{2}=\frac{\sqrt{\pi}}{4} \sigma_{N}^{2} \frac{k_{o}^{2} h_{\|}^{2} \sin ^{4} \zeta_{0}}{a \chi}\left[\frac{v^{2}}{\left(1-v \cos ^{2} \zeta_{0}\right)^{4} G_{0}} \equiv \beta\right] e^{\left(\frac{J}{a G_{0}}\right)^{2}}
$$

This expression is valid for arbitrary location of a point source and the receiver with respect to scattered layer [80].

Because we are looking forward to obtaining the phase variance as a function of the frequency we need to make the following simplification of $\alpha$ by substituting (163) and (169) into (171)

$$
\alpha=k_{0}^{2}\left[\alpha_{0} \equiv \frac{1}{2 N_{0}} \frac{v \sin ^{2} \zeta_{0}}{\left(1-v \cos ^{2} \zeta_{0}\right)^{2}}\right] \cong \frac{1}{2 N_{0}} \frac{\omega_{p}^{2} \sin ^{2} \zeta_{0}}{c^{2}}
$$

Similarly, we simplify $\beta$ and $J / G_{0}$ by substituting (169) into (182) and then (169) and (182) into (184) and assuming that $\omega \gg \omega_{p}$ yields

$$
\begin{gathered}
\beta=\frac{\omega_{p}^{4} / \omega^{4}}{\left(1-\frac{\omega_{p}^{2} \cos ^{2} \theta_{0}}{\omega^{2}}\right)^{4}\left(a_{1}+a_{2} \frac{\omega_{p}^{4}}{\omega^{4}} \gamma^{2}-2 a_{3} \frac{\omega_{p}^{2}}{\omega^{2}} \gamma\right)} \cong \frac{1}{\omega^{4}}\left(\frac{\omega_{p}^{4}}{a_{1}^{\frac{3}{2}}} \equiv \beta_{1}\right) \\
\frac{J}{G_{0}}=\frac{2 s J_{0}}{a_{1}+a_{2} A^{2}-2 a_{3} A}=\frac{2 \frac{v_{\text {eff }} \omega_{p}^{2}}{\omega \omega^{2}} \delta}{a_{1}+a_{2} \frac{\omega_{p}^{4}}{\omega^{4}} \gamma^{2}-2 a_{3} \frac{\omega_{p}^{2}}{\omega^{2}} \gamma} \cong \frac{\frac{2 v_{\mathrm{eff}} \omega_{p}^{2} \delta}{a_{1}} \equiv \varepsilon}{\omega^{3}}
\end{gathered}
$$

Hence, substituting (186) and (187) into (184) yields an approximation equal to

$$
\sigma_{\varphi}^{2} \cong \frac{\sqrt{\pi}}{4} \sigma_{N}^{2} \frac{k_{0}^{2} h_{\|}^{2} \sin ^{4} \zeta_{0}}{a \chi} \frac{\widetilde{\beta}_{1}}{\omega^{4}} e^{\frac{\varepsilon^{2}}{a^{2} \omega^{6}}}
$$

For the isotropic case $\chi=1$ and ignoring the influence of the geometric parameters we obtain the main component of the phase variance as a function of the signal frequency

$$
\sigma_{\varphi}^{2} \approx \frac{\widetilde{\beta}_{1}}{\omega^{2}} e^{\frac{\varepsilon^{2}}{\omega^{6}}}
$$

If the Satellite Transmitter and the Receiver are located on the upper and lower boundaries of plane plasma layer then we have $b=d=0$, in isotropic case, $\chi=1$, at $\zeta_{0}=90^{\circ}$, then (184) becomes

$$
\sigma_{\varphi}^{2}=\sqrt{\pi} \sigma_{N}^{2} h_{\|} h_{2}\left(\frac{k_{o}^{2} v^{2}}{4} \approx \frac{\frac{\omega^{2} \omega_{p}^{2}}{c^{2} \omega^{2}}}{4}=\frac{\frac{\omega_{p}^{2}}{c^{2}}}{4}=\frac{2 \pi^{2} a n_{e}}{c^{2}}\right)
$$

This is an upper bound of the phase variance as it is invariant of the frequency.

In Jandieri et al, 2007 [92] and 2008, [93] the phase variance $\left\langle\varphi_{1}^{2}\right\rangle$ and intensity scintillation index $S_{4}$ are related as follows

$$
\left\langle\varphi_{1}^{2}\right\rangle=\sqrt{\pi} \sigma_{N}^{2}\left(D^{2}+E^{2}\right) \frac{k_{0}^{2} l_{\|} Z}{Q \chi}
$$

$S_{4}^{2}=2\left\langle\varphi_{1}^{2}\right\rangle G^{\mathrm{xvi}}$

$G=1-\frac{\cos \left\{\frac{\tan ^{-1}\left(2 k_{\perp}^{2} / k_{f}^{2}\right)+\tan ^{-1}\left[2 k_{\perp}^{2} /\left(Q^{2} \chi^{2} k_{f}^{2}\right)\right]}{2}\right\}}{\left(1+4 k_{\perp}^{4} / k_{f}^{4}\right)^{\frac{1}{4}}\left[1+4 k_{\perp}^{4} /\left(Q^{4} \chi^{4} k_{f}^{4}\right)\right]^{\frac{1}{4}}}$

$Q^{2}(\alpha, \chi)=\frac{1+\chi^{2} w^{4}}{z^{2}}+b^{2} \frac{z^{2}}{\chi^{2}}-2 b w^{2}$

$D=\frac{A_{v}^{\prime}}{a_{z}^{\prime}}$ 
$D=D_{1}+D_{2}+D_{3}$

$D_{1}=\frac{\varepsilon^{\prime}\left(\eta^{\prime}-n_{1}^{2}\right)\left(\eta^{\prime}-1\right)-\mu^{\prime \prime 2}\left(\varepsilon^{\prime}-n_{1}^{2} \sin ^{2} \zeta_{1}\right)}{N_{*}\left[2 \varepsilon^{\prime}\left(\eta^{\prime}-n_{1}^{2}\right)+n_{1}^{2}\left(\varepsilon^{\prime}-\eta^{\prime}\right) \sin ^{2} \zeta\right] \cos \zeta_{1}}$

$D_{2}=\frac{n_{1}^{2}\left(\eta^{\prime}-1\right)\left(n_{1}^{2}-2 \eta^{\prime}+\varepsilon^{\prime}\right) \sin ^{2} \zeta_{1}}{2 N_{*}\left[2 \varepsilon^{\prime}\left(\eta^{\prime}-n_{1}^{2}\right)+n_{1}^{2}\left(\varepsilon^{\prime}-\eta^{\prime}\right) \sin ^{2} \zeta\right] \cos \zeta_{1}}$

$D_{3}=\frac{\left(n_{1}^{2}-\eta^{\prime}\right)\left(n_{1}^{2} \cos ^{2} \zeta_{1}-\eta^{\prime}\right)\left(\varepsilon^{\prime}-1\right)-\mu^{\prime \prime 2}\left(\varepsilon^{\prime}-1\right)}{2 N_{*}\left[2 \varepsilon^{\prime}\left(\eta^{\prime}-n_{1}^{2}\right)+n_{1}^{2}\left(\varepsilon^{\prime}-\eta^{\prime}\right) \sin ^{2} \zeta\right] \cos \zeta_{1}}$

$b=\frac{a_{y}^{\prime}}{a_{z}^{\prime}}$

$$
\begin{aligned}
& =\frac{N_{*}\left[\left(\eta^{\prime}-n_{1}^{2}\right)\left(\eta^{\prime}+\varepsilon^{\prime}\right)+n_{1}^{2}\left(\varepsilon^{\prime}-\eta^{\prime}\right) \sin ^{2} \zeta_{1}-\mu^{\prime \prime 2}\right] \sin \zeta_{1}}{N_{*}\left[2 \varepsilon^{\prime}\left(\eta^{\prime}-n_{1}^{2}\right)+n_{1}^{2}\left(\varepsilon^{\prime}-\eta^{\prime}\right) \sin ^{2} \zeta_{1}\right] \cos \zeta_{1}} \\
& =\frac{\left[\left(\eta^{\prime}-n_{1}^{2}\right)\left(\eta^{\prime}+\varepsilon^{\prime}\right)+n_{1}^{2}\left(\varepsilon^{\prime}-\eta^{\prime}\right) \sin ^{2} \zeta_{1}-\mu^{\prime \prime 2}\right] \tan \zeta_{1}}{2 \varepsilon^{\prime}\left(\eta^{\prime}-n_{1}^{2}\right)+n_{1}^{2}\left(\varepsilon^{\prime}-\eta^{\prime}\right) \sin ^{2} \zeta_{1}}
\end{aligned}
$$

Where

$$
\begin{aligned}
& z^{2}=\sin ^{2} \alpha+\chi^{2} \cos ^{2} \alpha \\
& w^{2}=\frac{\chi^{2}-1}{\chi^{2}} \sin \alpha \cos \alpha \\
& \eta^{\prime}=1-\frac{v\left(1+s^{2}-u\right)}{\left(1-s^{2}-u\right)^{2}+4 s^{2}} \\
& \eta^{\prime \prime}=\frac{s v\left(1+s^{2}-u\right)}{\left(1-s^{2}-u\right)^{2}+4 s^{2}} \\
& \varepsilon^{\prime}=1-\frac{v}{1+s^{2}} \\
& \varepsilon^{\prime \prime}=\frac{s v}{1+s^{2}} \\
& \mu^{\prime}=\frac{2 s v \sqrt{u}}{\left(1+s^{2}-u\right)^{2}+4 s^{2}} \\
& \mu^{\prime \prime}=\frac{v \sqrt{u}\left(1-s^{2}-u\right)}{\left(1+s^{2}-u\right)^{2}+4 s^{2}} \\
& N_{*}^{2}=\frac{n_{1}^{2} \pm \sqrt{n_{1}^{4}-n_{2}^{2}}}{2} \\
& n_{1}^{2}=1-2 v \frac{(1-v)\left[2\left(1-v-s^{2}\right)-u \sin ^{2} \zeta_{1} \pm P\right]-s[2 s(v-2) \pm U]}{\left[2\left(1-v-s^{2}\right)-u \sin ^{2} \zeta_{1} \pm P\right]^{2}+[2 s(v-2) \pm U]^{2}} \\
& n_{2}^{2}=-2 v \frac{(1-v)[2 s(v-2) \pm U]+s\left[2\left(1-v-s^{2}\right)-u \sin ^{2} \zeta_{1} \pm P\right]}{\left[2\left(1-v-s^{2}\right)-u \sin ^{2} \zeta_{1} \pm P\right]^{2}+[2 s(v-2) \pm U]^{2}} \\
& P=\sqrt{0.5\left(\sqrt{p^{2}+q^{2}}+p\right)}
\end{aligned}
$$

Where

$$
\begin{aligned}
& A_{v}^{\prime \prime}=A_{v 1}^{\prime \prime}+A_{v 2}^{\prime \prime}+A_{v 3}^{\prime \prime} \\
& A_{v i}^{\prime \prime}=A_{v i 1}^{\prime \prime}+A_{v i 2}^{\prime \prime}+A_{v i 3}^{\prime \prime} ; i=\{1,2,3\} \\
& A_{v 11}^{\prime \prime}=\left[\varepsilon^{\prime}\left(n_{2}^{2}-\eta^{\prime \prime}\right)-\varepsilon^{\prime \prime}\left(\eta^{\prime}-n_{1}^{2}\right)\right]\left(\eta^{\prime}-1\right) \\
& A_{v 12}^{\prime \prime}=-\varepsilon^{\prime}\left(\eta^{\prime}-n_{1}^{2}\right) \eta^{\prime \prime}-\mu^{\prime \prime 2}\left(n_{2}^{2} \sin ^{2} \zeta_{1}-\varepsilon^{\prime \prime}\right) \\
& A_{v 13}^{\prime \prime}=-2 \mu^{\prime} \mu^{\prime \prime}\left(\varepsilon^{\prime}-n_{1}^{2} \sin ^{2} \zeta_{1}\right) \\
& A_{v 21}^{\prime \prime}=-\frac{n_{1}^{2}\left(\eta^{\prime}-1\right)\left(n_{2}^{2}-2 \eta^{\prime \prime}+\varepsilon^{\prime \prime}\right) \sin ^{2} \zeta_{1}}{2}
\end{aligned}
$$

$A_{v 22}^{\prime \prime}=-\frac{\left[n_{2}^{2}\left(\eta^{\prime}-1\right)+n_{1}^{2} \eta^{\prime \prime}\right]\left(n_{1}^{2}-2 \eta^{\prime \prime}+\varepsilon^{\prime}\right) \sin ^{2} \zeta_{1}}{2}$

$A_{v 23}^{\prime \prime}=-\frac{\left(n_{1}^{2}-\eta^{\prime}\right)\left(n_{1}^{2} \cos ^{2} \zeta_{1}-\eta^{\prime}\right) \varepsilon^{\prime \prime}}{2}$

$A_{v 31}^{\prime \prime}=-\frac{\left(\varepsilon^{\prime}-1\right)\left(n_{1}^{2}-\eta^{\prime}\right)\left(\eta^{\prime \prime}-n_{2}^{2} \cos ^{2} \zeta_{1}\right)}{2}$

$A_{v 32}^{\prime \prime}=-\frac{\left(\varepsilon^{\prime}-1\right)\left(\eta^{\prime \prime}-n_{2}^{2}\right)\left(n_{1}^{2} \cos ^{2} \zeta_{1}-\eta^{\prime}\right)}{2}$

$A_{v 33}^{\prime \prime}=-\frac{2 \mu^{\prime} \mu^{\prime \prime}\left(\varepsilon^{\prime}-1\right)-\varepsilon^{\prime \prime} \mu^{\prime \prime 2}}{2}$

$a_{z}^{\prime \prime}=a_{z 1}^{\prime \prime}+a_{z 2}^{\prime \prime}$

$a_{z 1}^{\prime \prime}=2 N_{*}\left[\varepsilon^{\prime}\left(n_{2}^{2}-\eta^{\prime \prime}\right)-\varepsilon^{\prime \prime}\left(\eta^{\prime}-n_{1}^{2}\right)\right] \cos \zeta_{1}$

$a_{z 2}^{\prime \prime}=N_{*}\left[n_{1}^{2}\left(\eta^{\prime \prime}-\varepsilon^{\prime \prime}\right)-n_{2}^{2}\left(\varepsilon^{\prime}-\eta^{\prime}\right)\right] \sin ^{2} \zeta_{1} \cos \zeta_{1}$ 


$$
\begin{aligned}
& k_{\perp}^{2}=k_{f}^{2} \frac{\chi^{2}}{h_{\|}^{2}} \frac{\lambda z}{\pi} \\
& k_{\perp}=k_{f} \frac{\chi}{h_{\|}} \sqrt{\frac{\lambda z}{\pi}}=k_{f} \frac{h_{\|}}{h_{\|} h_{\perp}} \sqrt{\frac{c z}{f \pi}}=\frac{k_{f}}{h_{\perp}} \sqrt{\frac{c z}{f \pi}}
\end{aligned}
$$

Next, we consider a special case: For turbulent collision-less magnetized plasma $s=0$ and assuming that $\zeta=0^{\circ}$.

Furthermore, let assume case (a) magnetic field influence is zero, $u=0$; hence, we obtain the following

$$
\eta^{\prime}=1-\frac{v(1-u)}{(1-u)^{2}}=1-v
$$

This concludes the description of the amplitude and phase scintillation indexes.

\section{Description of a simplified ionospheric channel model}

The exact computation of the ionospheric channel model based on the equations presented previously is a daunting task, because the results will depend on the profile of $v_{i}(h)$ which is obtained from measurements; hence, we consider two simplified examples of $v_{i}(h)$ profiles.

\subsection{Examples of description of simplified ionospheric delay}

Example 1: In the first example we assume that $v_{i}(h)$ is given by

$$
v_{i}(h)=\mid \begin{array}{ll}
v_{1 i}\left[1-\mathrm{e}^{-\alpha_{i}\left(h-h_{0}\right)}\right], & h_{0} \leq h \leq h_{i} \\
v_{2 i}\left[\mathrm{e}^{-\beta_{i}\left(h-h_{1}\right)}-1\right], & h_{i} \leq h \leq h_{1}
\end{array}
$$

Where $i=1 \equiv N S$ and $i=2 \equiv E W$.

First, let us compute the integral $I$

$$
\begin{aligned}
I & =\int_{h_{0}}^{\infty} v_{i}(h) h d h \\
& =I_{1}+I_{2}
\end{aligned}
$$

as a sum of two integrals: $I_{1}$ and $I_{2}$

$$
\begin{aligned}
I_{1} & =\int_{h_{0}}^{h_{i}} v_{1 i}\left[1-e^{-\alpha_{i}\left(h-h_{0}\right)}\right] h d h \\
& =v_{1 i} \int_{h_{0}}^{h_{i}} h d h-v_{1 i} e^{\alpha_{i} h_{0}} \int_{h_{0}}^{h_{i}} e^{-\alpha_{i} h} h d h \\
& =v_{1 i}\left\{\left.\frac{h^{2}}{2}\right|_{h_{0}} ^{h_{i}}+e^{\alpha_{i} h_{0}}\left[\left.\frac{h \mathrm{e}^{-\alpha_{i} h}}{\alpha_{i}}\right|_{h_{0}} ^{h_{i}}-\left.\frac{\mathrm{e}^{-\alpha_{i} h}}{\alpha_{i}^{2}}\right|_{h_{0}} ^{h_{i}}\right]\right\} \\
& =v_{1 i}\left[\frac{h_{i}^{2}-h_{0}^{2}}{2}+\frac{h_{i} e^{-\alpha_{i}\left(h_{i}-h_{0}\right)_{-}}}{\alpha_{i}}+\frac{1-e^{-\alpha_{i}\left(h_{i}-h_{0}\right)}}{\alpha_{i}^{2}}\right] \\
I_{2} & =\int_{h_{i}}^{h_{1}} v_{2 i}\left[e^{-\beta_{i}\left(h-h_{1}\right)}-1\right] h d h
\end{aligned}
$$

$$
\begin{aligned}
& =v_{2 i} e^{\beta_{i} h_{1}} \int_{h_{i}}^{h_{1}} e^{-\beta_{i} h} h d h-v_{2 i} \int_{h_{i}}^{h_{1}} h d h \\
& =v_{2 i}\left\{e^{\beta_{i} h_{1}}\left[-\left.\frac{h e^{-\beta_{i} h}}{\beta_{i}}\right|_{h_{i}} ^{h_{1}}+\left.\frac{e^{-\beta_{i} h}}{\beta_{i}^{2}}\right|_{h_{i}} ^{h_{1}}\right]-\left.\frac{h^{2}}{2}\right|_{h_{i}} ^{h_{1}}\right\} \\
& =v_{2 i}\left[\frac{h_{i} e^{-\beta_{i}\left(h_{1}-h_{i}\right)}-h_{1}}{\beta_{i}}+\frac{1-e^{-\beta_{i}\left(h_{1}-h_{i}\right)}}{\beta_{i}^{2}}+\frac{h_{i}^{2}-h_{1}^{2}}{2}\right]
\end{aligned}
$$

Combining, (240) and (241) into (239) the following is obtained

$$
v_{i}(h)=v_{1 i} a_{1 i}+v_{2 i} a_{2 i}
$$

Where

$$
\begin{aligned}
& a_{1 i}=\frac{h_{i}^{2}-h_{0}^{2}}{2}+\frac{h_{i} e^{-\alpha_{i}\left(h_{i}-h_{0}\right)}-h_{0}}{\alpha_{i}}+\frac{1-e^{-\alpha_{i}\left(h_{i}-h_{0}\right)}}{\alpha_{i}^{2}} \\
& a_{2 i}=\frac{h_{i} e^{-\beta_{i}\left(h_{1}-h_{i}\right)}-h_{1}}{\beta_{i}}+\frac{1-e^{-\beta_{i}\left(h_{1}-h_{i}\right)}}{\beta_{i}^{2}}+\frac{h_{i}^{2}-h_{1}^{2}}{2}
\end{aligned}
$$

This can be further simplified as because the equality due to the continuity of the IP point

$$
v_{\mathrm{m} i}=v_{1 i}\left[1-e^{-\alpha_{i}\left(h_{i}-h_{0}\right)}\right]=v_{2 i}\left[e^{-\beta_{i}\left(h_{i}-h_{1}\right)}-1\right]
$$

Hence, since

$$
\begin{gathered}
\int_{h_{0}}^{\infty} v_{i}(h) h d h=\left.\frac{\partial T E C}{\partial x_{i}}\right|_{x_{i}=0} \\
=c_{i 1}(t)+c_{i 2}(t)+c_{i 3}(t) \\
c_{i 1}(t)=\frac{h_{i}^{2}(t)\left[v_{1 i}(t)+v_{2 i}(t)\right]-h_{0}^{2}(t) v_{1 i}(t)-h_{1}^{2}(t) v_{2 i}(t)}{2} \\
c_{i 2}(t)=\frac{v_{\mathrm{m} i}(t)}{\alpha_{i}^{2}(t)}-\frac{v_{\mathrm{m} i}(t)}{\beta_{i}^{2}(t)} \\
c_{i 3}(t)=c_{i 31}(t)+c_{i 32}(t) \\
c_{i 31}(t)=\frac{h_{i}(t)\left[v_{\mathrm{m} i}(t)+v_{2 i}(t)\right]-h_{1}(t) v_{2 i}(t)}{\beta_{i}(t)} \\
c_{i 32}(t)=\frac{h_{i}(t)\left[v_{1 i}(t)-v_{\mathrm{m} i}(t)\right]-h_{0}(t) v_{1 i}(t)}{\alpha_{i}(t)}
\end{gathered}
$$

This concludes the derivations of Example 1.

Example 2: In the second example we assume that $v_{i}(h)$ is given by

$$
v_{i}(h)=\mid \begin{array}{cc}
v_{1 i}\left[e^{\alpha_{i}\left(h-h_{0}\right)}-1\right], & h_{0} \leq h \leq h_{i} \\
v_{2 i}\left[e^{-\beta_{i}\left(h-h_{1}\right)}-1\right], & h_{i} \leq h \leq h_{1}
\end{array}
$$

Where $i=1 \equiv N S$ and $i=2 \equiv E W$.

First, let us compute the integral $I$

$$
\begin{aligned}
I & =\int_{h_{0}}^{\infty} v_{i}(h) h d h \\
& =I_{1}+I_{2}
\end{aligned}
$$

as a sum of two integrals: $I_{1}$ and $I_{2}$ (same as (241)) 


$$
\begin{aligned}
I_{1} & =\int_{h_{0}}^{h_{i}} v_{1 i}\left[e^{\alpha_{i}\left(h-h_{0}\right)}-1\right] h d h \\
& =v_{1 i} e^{-\alpha_{i} h_{0}} \int_{h_{0}}^{h_{i}} e^{\alpha_{i} h} h d h-v_{1 i} \int_{h_{0}}^{h_{i}} h d h \\
& =v_{1 i}\left\{e^{-\alpha_{i} h_{0}}\left[\left.\frac{h \mathrm{e}^{\alpha_{i} h}}{\alpha_{i}}\right|_{h_{0}} ^{h_{i}}-\left.\frac{\mathrm{e}^{\alpha_{i} h}}{\alpha_{i}^{2}}\right|_{h_{0}} ^{h_{i}}\right]-\left.\frac{h^{2}}{2}\right|_{h_{0}} ^{h_{i}}\right\} \\
& =v_{1 i}\left[\frac{h_{i} e^{\alpha_{i}\left(h_{i}-h_{0}\right)}-h_{0}}{\alpha_{i}}+\frac{1-e^{\alpha_{i}\left(h_{i}-h_{0}\right)}}{\alpha_{i}^{2}}+\frac{h_{0}^{2}-h_{i}^{2}}{2}\right]
\end{aligned}
$$

Combining, (254) and (241) into (153) the following is obtained

$$
\int_{h_{0}}^{\infty} v_{i}(h) h d h=v_{1 i} a_{1 i}+v_{2 i} a_{2 i}
$$

Where $a_{2 i}$ (the same as (244))

$$
a_{1 i}=\frac{h_{i} e^{\alpha_{i}\left(h_{i}-h_{0}\right)}-h_{0}}{\alpha_{i}}+\frac{1-e^{\alpha_{i}\left(h_{i}-h_{0}\right)}}{\alpha_{i}^{2}}+\frac{h_{0}^{2}-h_{i}^{2}}{2}
$$

This can be further simplified as because the equality due to the continuity of the IP point

$$
v_{\mathrm{m} i}=v_{1 i}\left[e^{\alpha_{i}\left(h_{i}-h_{0}\right)}-1\right]=v_{2 i}\left[e^{-\beta_{i}\left(h_{i}-h_{1}\right)}-1\right]
$$

Hence, since

$$
\begin{gathered}
\int_{h_{0}}^{\infty} v_{i}(h) h d h=\left.\frac{\partial T E C}{\partial x_{i}}\right|_{x_{i}=0} \\
=c_{i 1}(t)+c_{i 2}(t)+c_{i 3}(t) \\
c_{i 1}(t)=\frac{h_{i}^{2}(t)\left[v_{1 i}(t)+v_{2 i}(t)\right]-h_{0}^{2}(t) v_{1 i}(t)-h_{1}^{2}(t) v_{2 i}(t)}{2} \\
c_{i 2}(t)=-\frac{v_{\mathrm{m} i}(t)}{\alpha_{i}^{2}(t)}-\frac{v_{\mathrm{m} i}(t)}{\beta_{i}^{2}(t)} \\
c_{i 3}(t)=c_{i 31}(t)+c_{i 32}(t) \\
c_{i 31}(t)=\frac{h_{i}(t)\left[v_{\mathrm{m} i}(t)+v_{2 i}(t)\right]-h_{1}(t) v_{2 i}(t)}{\beta_{i}(t)} \\
c_{i 32}(t)=\frac{h_{i}(t)\left[v_{1 i}(t)+v_{\mathrm{m} i}(t)\right]-h_{0}(t) v_{1 i}(t)}{\alpha_{i}(t)}
\end{gathered}
$$

In order to understand the relationship between the two profiles let us consider further that $v_{\mathrm{m} i}$ given by (257) is the same as $v_{\mathrm{m} i}$ given by (245); or

$$
\begin{aligned}
& v_{\mathrm{m} i}=v_{1 i(1)}\left[1-e^{-\alpha_{i(1)}\left(h_{i}-h_{0}\right)}\right] \\
& v_{\mathrm{m} i}=v_{1 i(2)}\left[e^{\alpha_{i(2)}\left(h_{i}-h_{0}\right)}-1\right]
\end{aligned}
$$

Which is equivalent with

$$
\begin{aligned}
& v_{1 i(1)}=\frac{v_{\mathrm{m} i}}{1-e^{-\alpha_{i(1)}\left(h_{i}-h_{0}\right)}} \\
& v_{1 i(2)}=\frac{v_{\mathrm{m} i}}{e^{\alpha_{i(2)}\left(h_{i}-h_{0}\right)}-1}
\end{aligned}
$$

One then can utilize these calculations to compute a simplified total ionospheric delay based on the equations that were previously presented in this paper.

\subsection{Examples of description of the simplified ionospheric Doppler frequency}

In the following two examples we will utilize exactly the same simplified profile that we utilized in simplified ionospheric delay.

Example 1: Assuming the first profile of the azimuth gradient is given by (246)-(251); hence, $\frac{d c_{i}(t)}{d t}$ can be obtained from the derivative of three expressions:

$$
\frac{d c_{i}(t)}{d t}=\frac{d c_{i 1}(t)}{d t}+\frac{d c_{i 2}(t)}{d t}+\frac{d c_{i 3}(t)}{d t}
$$

The first derivative, $\frac{d c_{i 1}(t)}{d t}$, can be computed from

$$
\frac{d c_{i 1}(t)}{d t}=\sum_{j=1}^{2}\left[a_{v_{j i}} v_{j i}(t)+a_{\dot{v}_{j i}} \frac{d v_{j i}(t)}{d t}\right]
$$

Where

$$
\begin{aligned}
& a_{v_{1 i}}=h_{i}(t) \frac{d h_{i}(t)}{d t}-h_{0}(t) \frac{d h_{0}(t)}{d t} \\
& a_{v_{2 i}}=h_{i}(t) \frac{d h_{i}(t)}{d t}-h_{1}(t) \frac{d h_{1}(t)}{d t} \\
& a_{\dot{v}_{1 i}}=\frac{h_{i}^{2}(t)-h_{0}^{2}(t)}{2} \\
& a_{\dot{v}_{2 i}}=\frac{h_{i}^{2}(t)-h_{1}^{2}(t)}{2}
\end{aligned}
$$

The second derivative, $\frac{d c_{i 2}(t)}{d t}$, can be computed from

$$
\frac{d c_{i 2}(t)}{d t}=a_{v_{\mathrm{m} i}} v_{\mathrm{m} i}(t)+a_{\dot{v}_{\mathrm{m} i}} \frac{d v_{\mathrm{m} i}(t)}{d t}
$$

Where

$$
\begin{aligned}
& a_{v_{\mathrm{m} i}}=2\left[\frac{d \beta_{i}(t)}{d t} \frac{1}{\beta_{i}^{3}(t)}-\frac{d \alpha_{i}(t)}{d t} \frac{1}{\alpha_{i}^{3}(t)}\right] \\
& a_{\dot{v}_{\mathrm{m} i}}=\frac{1}{\alpha_{i}^{2}(t)}-\frac{1}{\beta_{i}^{2}(t)}
\end{aligned}
$$

The third derivative, $\frac{d c_{i 3}(t)}{d t}$, can be computed from

$$
\frac{d c_{i 3}(t)}{d t}=a_{\beta_{i}}+a_{\dot{\beta}_{i}} \frac{d \beta_{i}(t)}{d t}+a_{\alpha_{i}}+a_{\dot{\alpha}_{i}} \frac{d \alpha_{i}(t)}{d t}
$$

Where 


$$
\begin{aligned}
& a_{\beta_{i}}=a_{\beta_{i 1}}+a_{\beta_{i 2}}+a_{\beta_{i 3}} \\
& a_{\beta_{i 1}}=\frac{\left[v_{\mathrm{m} i}(t)+v_{2 i}(t)\right]}{\beta_{i}(t)} \frac{d h_{i}(t)}{d t} \\
& a_{\beta_{i 2}}=\frac{1}{\beta_{i}(t)} \frac{h_{i}(t) d\left[v_{\mathrm{m} i}(t)+v_{2 i}(t)\right]}{d t} \\
& a_{\beta_{i 3}}=\frac{1}{\beta_{i}(t)} \frac{-d h_{1}(t) v_{2 i}(t)-h_{1}(t) v_{2 i}(t)}{d t} \\
& a_{\alpha_{i}}=a_{\alpha_{i 1}}+a_{\alpha_{i 2}}+a_{\alpha_{i 3}} \\
& a_{\alpha_{i 1}}=\frac{\left[v_{1 i}(t)-v_{\mathrm{m} i}(t)\right]}{\alpha_{i}(t)} \frac{d h_{i}(t)}{d t} \\
& a_{\alpha_{i 2}}=\frac{h_{i}(t)}{\alpha_{i}(t)}\left[\frac{d v_{1 i}(t)}{d t}-\frac{d v_{\mathrm{m} i}(t)}{d t}\right] \\
& a_{\alpha_{i 3}}=\frac{-1}{\alpha_{i}(t)}\left[\frac{d h_{0}(t) v_{1 i}(t)}{d t}+\frac{h_{0}(t) d v_{1 i}(t)}{d t}\right] \\
& a_{\beta_{i}}=\frac{h_{1}(t) v_{2 i}(t)-h_{i}(t)\left[v_{\mathrm{m} i}(t)+v_{2 i}(t)\right]}{\beta_{i}^{2}(t)} \\
& \alpha_{i}^{2}(t)
\end{aligned}
$$

Hence, substituting (269), (274), and (277) into (268) yields,

$$
\frac{d c_{i}(t)}{d t}=\sum_{\dot{k}} b_{\dot{k}} \dot{k}
$$

Where

$$
\begin{aligned}
& k=\left\{h_{0}, h_{1}, h_{i}, \alpha_{i}, \beta_{i}, v_{1 i}, v_{2 i}, v_{\mathrm{m} i}\right\} \\
& \dot{k}=\frac{d k(t)}{d t}=\left\{\dot{h}_{0}, \dot{h}_{1}, \dot{h}_{i}, \dot{\alpha}_{i}, \dot{\beta}_{i}, \dot{v}_{1 i}, \dot{v}_{2 i}, \dot{v}_{\mathrm{m} i}\right\} \\
& b_{\dot{h}_{0}}=-v_{1 i}(t)\left[h_{0}(t)+\frac{1}{\alpha_{i}(t)}\right] \\
& b_{\dot{h}_{1}}=-v_{2 i}(t)\left[h_{1}(t)+\frac{1}{\beta_{i}(t)}\right] \\
& b_{\dot{h}_{i}}=\frac{h_{i}(t)}{\left[v_{1 i}(t)+v_{2 i}(t)\right]^{-1}}+\frac{v_{\mathrm{m} i}(t)+v_{2 i}(t)}{\beta_{i}(t)}+\frac{v_{1 i}(t)-v_{\mathrm{m} i}(t)}{\alpha_{i}(t)} \\
& b_{\dot{\alpha}_{i}}=\frac{h_{i}(t)\left[v_{1 i}(t)-v_{\mathrm{m} i}(t)\right]-h_{0}(t) v_{1 i}(t)}{\alpha_{i}^{2}(t)}+\frac{2 v_{\mathrm{m} i}(t)}{\alpha_{i}^{3}(t)} \\
& b_{\dot{\beta}_{i}}=\frac{h_{I P}(t)\left[v_{\mathrm{m} i}(t)+v_{2 i}(t)\right]-h_{1}(t) v_{2 i}(t)}{\beta_{i}^{2}(t)}-\frac{2 v_{\mathrm{m} i}(t)}{\beta_{i}^{3}(t)} \\
& b_{\dot{v}_{1 i}}=\frac{h_{i}^{2}(t)-h_{0}^{2}(t)}{2}+\frac{h_{i}(t)-h_{0}(t)}{\alpha_{i}(t)}
\end{aligned}
$$

$$
b_{\dot{v}_{\mathrm{m} i}}=\frac{1}{\beta_{i}^{2}(t)}-\frac{1}{\alpha_{i}^{2}(t)}+\frac{h_{i}(t)}{\beta_{i}(t)}+\frac{h_{i}(t)}{\alpha_{i}(t)}
$$

Example 2: Next, assuming the second profile of the azimuth gradient by (258)-(263).

Performing calculations which are very similar to example 1 one can obtain (288) where $k, \dot{k}, b_{\dot{h}_{1}}, b_{\dot{\beta}_{i}}$, and $b_{\dot{v}_{2 i}}$ are given by (289), (290), (292), (295), and (297) respectively and the other variables are computed as follows

$$
\begin{aligned}
& b_{\dot{h}_{0}}=v_{1 i}(t)\left[h_{0}(t)+\frac{1}{\alpha_{i}(t)}\right] \\
& b_{\dot{h}_{i}}=\frac{h_{i}(t)}{\left[v_{1 i}(t)+v_{2 i}(t)\right]^{-1}}+\frac{v_{\mathrm{m} i}(t)+v_{2 i}(t)}{\beta_{i}(t)}+\frac{v_{\mathrm{m} i}(t)-v_{1 i}(t)}{\alpha_{i}(t)} \\
& b_{\dot{\alpha}_{i}}=\frac{h_{0}(t) v_{1 i}(t)-h_{i}(t)\left[v_{\mathrm{m} i}(t)+v_{1 i}(t)\right]}{\alpha_{i}^{2}(t)}+\frac{2 v_{\mathrm{m} i}(t)}{\alpha_{i}^{3}(t)} \\
& b_{\dot{v}_{1 i}}=\frac{h_{i}^{2}(t)+h_{0}^{2}(t)}{2}+\frac{h_{0}(t)-h_{i}(t)}{\alpha_{i}(t)} \\
& b_{\dot{v}_{\mathrm{m} i}}=\frac{1}{\beta_{i}^{2}(t)}-\frac{1}{\alpha_{i}^{2}(t)}+\frac{h_{i}(t)}{\beta_{i}(t)}-\frac{h_{i}(t)}{\alpha_{i}(t)}
\end{aligned}
$$

One then can utilize these calculations to compute a simplified total ionospheric Doppler frequency based on the equations that were presented previously in this journal paper.

\section{Numerical, Theoretical Results}

Example 1: In Figs. 13-16 the following are depicted: (1) the ionospheric gradient PED profile vs. the height of the ionosphere and (2) the obliquity factor $m_{i}(t)$ (or $\left.m_{i}(t)\right)$ and the azimuth obliquity factor $m_{i}^{a z}(t)$ (or $m_{i}^{a z}[\theta(t)]$ ) as a function of elevation $\theta(t)$ (or zenith $\zeta(t)$ ) angle. First, the ionospheric gradient PED profile of the first or the second scenario increases monotonically from $0 / 0$ els $/ \mathrm{m}^{2}$ to $60 / 96$ Mels $/ \mathrm{m}^{2}$ as a function of height from $50 \mathrm{~km}$ to $350 \mathrm{~km}$ in EastWest(EW)/NorthSouth(NS) and then decreases monotonically from $60 / 96 \mathrm{Mels} / \mathrm{m}^{2}$ to $0 / 0 \mathrm{els} / \mathrm{m}^{2}$ as a function of height from $350 \mathrm{~km}$ to $1000 \mathrm{~km}$ from EW/NS. In either profile the maximum ionospheric gradient PED profile 60/96 Mels $/ \mathrm{m}^{2}$ in $\mathrm{EW} / \mathrm{NS}$ corresponds to the ionospheric height of $350 \mathrm{~km}$. Now this height can change and so can the maximum value of the ionospheric gradient PED profile in the simulation setting. 
As can be seen from Figs. 13-16, gradients become increasingly influential at lower elevation angles.

Example 2: The second simulation example is the illustration of the RF L-band (1-2 GHz) ionospheric propagation channel model in Figs. 17-20. This basic simulation contains the description of: (1) refractive index [5], $n$; (2) refractivity, $N$; (3) ionospheric zenith delay, $D_{i}^{z}[m]$ and (4) ionospheric azimuth critical zenith delay, $D_{i}^{a z, z c}[m]$ as function of frequency $1 \mathrm{GHz} \leq f \leq 2 \mathrm{GHz}$. By taking a look at the plots in Figs. 17-19 we observe that refractivity index and refractivity/ionospheric delay [11] increase/decrease monotonically for values of frequency $1 \mathrm{GHz} \leq f \leq 2 \mathrm{GHz}$.

The smallest refractive index [5] 0.9999596918069695 or the smallest (largest in magnitude) refractivity -40 corresponds to values of $f=1 \mathrm{GHz}$ and the largest refractive index [5] 0.9999900229517423 or the largest (smallest in magnitude) refractivity -10 correspond to values of $f=$ $2 \mathrm{GHz}$. The ionospheric zenith delay decreases from $16 \mathrm{~m}$ at $1 \mathrm{GHz}$ to $4 \mathrm{~m}$ at $2 \mathrm{GHz}$ and the ionospheric azimuth critical zenith delay decreases from $8.5 \mathrm{~m}$ at $1 \mathrm{GHz}$ to $1 \mathrm{~m}$ at $2 \mathrm{GHz}$.

Example 3: The third simulation example is the illustration of the ionospheric Doppler frequency elements such as $\partial T E C / \partial x_{i}, d c_{i}(t) / d t, \alpha^{\prime}(t)$, and, $\beta(t)$ vs. time, zenith angle, $\zeta(t)$, is the sum of two angles $\alpha^{\prime}(t)$ with $\beta(t)$, and $\zeta^{\prime}(t)$, (see Figs. 21-24).

Example 4: The fourth example of a simulation of the RF Lband (1-2 GHz) ionospheric propagation channel model 2 is illustrated in Figs. 25-28.

This basic simulation contains the description of: (1) total ionospheric delay [11], $D_{i}^{a s}[\mathrm{~m}]$; (2) total ionospheric Doppler, $f_{i}^{D, a s}[\mathrm{MHz}]$; as a function of time of day [h] and of frequency $1 \mathrm{GHz} \leq f \leq 2 \mathrm{GHz}$ using the first envelope of the PED profile (see Fig. 13). By taking a look at the plots in Figs. 2528 we observe that total ionospheric delay [11] and total ionospheric Doppler increase/decrease monotonically as a function of time/of frequency $1 \mathrm{GHz} \leq f \leq 2 \mathrm{GHz}$. The smallest values of the total ionospheric delay [11] of $8.59 \mathrm{~m}$ $(f=1,176.45 \mathrm{MHz})$ and $4.79 \mathrm{~m}(f=1,575.42 \mathrm{MHz})$ correspond to $t=0 \mathrm{~h}$ at zenith or $(\zeta=0)$ and largest values of the total ionospheric delay [11] of $206 \mathrm{~m} \mathrm{(} f=$ $1,176.45 \mathrm{MHz})$ and $115 \mathrm{~m}(f=1,575.42 \mathrm{MHz})$ correspond to $t=2.52 \mathrm{~h}$ at the horizon or $\left(\zeta=90^{\circ}\right)$.

The smallest values of the total ionospheric Doppler of $-1.53 \mathrm{~Hz}(f=1,176.45 \mathrm{GHz})$ and $-1.14 \mathrm{~Hz} \quad(f=$ $1,575.42 \mathrm{MHz})$ correspond to $t=0 \mathrm{~h}$ at zenith or $(\zeta=0)$ and largest values of the total ionospheric Doppler of $121 \mathrm{~Hz}(f=$ $1,176.45 \mathrm{MHz})$ and $165 \mathrm{~Hz}(f=1,575.42 \mathrm{MHz})$ correspond to $t=2.52 \mathrm{~h}$ at the horizon or $\left(\zeta=90^{\circ}\right)$.

Example 5: The fifth example of a simulation of the RF Lband $(1-2 \mathrm{GHz})$ ionospheric propagation channel model 3 is illustrated in Figs. 29-32. This basic simulation contains the description of: (1) total ionospheric delay [11], $D_{i}^{a s}[\mathrm{~m}]$; (2) total ionospheric Doppler, $f_{i}^{D, a s}[\mathrm{MHz}]$; as a function of time of day [h] and of frequency $1 \mathrm{GHz} \leq f \leq 2 \mathrm{GHz}$ using the second envelope of the PED profile (see Fig. 14).

By taking a look at the plots in Figs. 29-32 we observe that total ionospheric delay [11] and total ionospheric Doppler increase/decrease monotonically as a function of time/of frequency $1 \mathrm{GHz} \leq f \leq 2 \mathrm{GHz}$.

The smallest values of the total ionospheric delay [11] of $8.59 \mathrm{~m} \quad(f=1,176.45 \mathrm{MHz})$ and $4.79 \mathrm{~m} \quad(f=$ $1,575.42 \mathrm{MHz})$ correspond to $t=0 \mathrm{~h}$ at zenith or $(\zeta=0)$ and largest values of the total ionospheric delay [11] of $183 \mathrm{~m}$ $(f=1,176.45 \mathrm{MHz})$ and $102 \mathrm{~m}(f=1,575.42 \mathrm{MHz})$ correspond to $t=2.52 \mathrm{~h}$ at the horizon or $\left(\zeta=90^{\circ}\right)$. The smallest values of the total ionospheric Doppler of $-1.53 \mathrm{~Hz}$ $(f=1,176.45 \mathrm{GHz})$ and $-1.14 \mathrm{~Hz}(f=1,575.42 \mathrm{MHz})$ correspond to $t=0 \mathrm{~h}$ at zenith or $(\zeta=0)$ and largest values of the total ionospheric Doppler of $72 \mathrm{~Hz}(f=1,176.45 \mathrm{MHz})$ and $53.7 \mathrm{~Hz}(f=1,575.42 \mathrm{MHz})$ correspond to $t=2.52 \mathrm{~h}$ at the horizon or $\left(\zeta=90^{\circ}\right)$.

Example 6: The sixth example of a simulation of the RF LBand (1-2 GHz) ionospheric scintillation amplitude, $S_{4}[\mu]$, and phase standard deviation, $\sigma_{\varphi}\left[^{\circ}\right]$, is illustrated in Figs. 3336. This basic simulation contains the description of: (1) ionospheric scintillation amplitude standard deviation, $S_{4}[\mu]$; (2) phase standard deviation, $\sigma_{\varphi}\left[{ }^{\circ}\right]$, as a function of frequency $1 \mathrm{GHz} \leq f \leq 2 \mathrm{GHz}$ having the PED, external magnetic field, and electron collision as parameters whose significance is given in sequential order.

By taking a look at the plots in Figs. 33-36 we observe that ionospheric scintillation amplitude, $S_{4}[\mu]$, and phase, $\sigma_{\varphi}\left[^{\circ}\right]$, standard deviation decreases monotonically as a function of frequency $1 \mathrm{GHz} \leq f \leq 2 \mathrm{GHz}$. On the top two plots: the largest/smallest values of $S_{4}$ are: 1300/276 [ $\left.\mu\right](f=$ $1.1765 \mathrm{GHz})$ and $761 / 154[\mu](f=1.547542 \mathrm{GHz})$ and $\sigma_{\varphi}\left[^{\circ}\right] \quad$ are: $27.2 / 3.55\left[^{\circ}\right] \quad(f=1.1765 \mathrm{GHz})$ and $21.4 / 2.65\left[^{\circ}\right](f=1.547542 \mathrm{GHz})$ correspond to $n_{e}=$ $\left[\begin{array}{ll}1 & 1\end{array}\right] \times 10^{12} \mathrm{el} / \mathrm{m}^{3} \quad, \quad B=\left[\begin{array}{ll}0 & 25 \times 10^{-4}\end{array}\right] \mathrm{T}, \quad \theta_{0}=$ $\left[\begin{array}{ll}0 & 89\end{array}\right]^{\circ}$, and $\mathrm{v}_{\mathrm{eff}}=\left[\begin{array}{ll}0 & 147.53\end{array}\right] \mathrm{Hz}$. 
On the bottom two plots: the largest/smallest values of $S_{4}$ are: $1300 / 249[\mu](f=1.1765 \mathrm{GHz})$ and $761 / 139[\mu]$ $(f=1.547542 \mathrm{GHz})$ and $\sigma_{\varphi}\left[^{\circ}\right]$ are: $27.2 / 3.19\left[^{\circ}\right] \quad(f=$ $1.1765 \mathrm{GHz})$ and $21.4 / 2.39\left[^{\circ}\right] \quad(f=1.547542 \mathrm{GHz})$ correspond to $n_{e}=\left[\begin{array}{ll}0.9 & 1\end{array}\right] \times 10^{12} \mathrm{el} / \mathrm{m}^{3}, \quad B=$ $\left[\begin{array}{ll}0 & 25 \times 10^{-4}\end{array}\right] \mathrm{T}, \quad \theta_{0}=\left[\begin{array}{ll}0 & 89\end{array}\right]^{\circ}, \quad$ and $\quad \mathrm{v}_{\mathrm{eff}}=$ [0 147.53 $] \mathrm{Hz}$.

An example amplitude variation and phase fluctuation of the basic scintillated component from the simulation are shown in [95]. The amplitude scintillation intensity is represented by the $S_{4}$ index which is defined as the normalized standard deviation of the signal power. The phase scintillation is characterized by the phase standard deviation. In the example shown in [95], the amplitude scintillation index $S_{4}$ is 1.01 and the phase scintillation standard deviation of 0.44 radians [96]. It is possible that some of the data is due to a 1-parameter ionospheric model used. The 4-parameter ionospheric model that is proposed in this paper should be able to present amplitude and phase scintillation data that are much lower (three to six orders of magnitude) than the ones in [95] or contrast with theoretical amplitude and phase scintillation depicted in Figs. 33-36.

I did however find a good reference from Abdullah et al. 2009 [97] that have employed a dual-frequency GPS data collected during the one-month ionospheric experimental campaign that was used for TEC and scintillation computation and analysis. The TEC with 15 sec interval were computed from combined L1 and L2 code-pseudorange and carrier phase measurements. Whereas, the scintillation parameter S4 index was computed as a standard deviation of the received signal power normalized to average signal power every $60 \mathrm{sec}$ on L1. A corrected $S_{4}$ (without noise effects) was also computed and used in the analysis.

Abdullah et al. 2009 [97] have reported daily maxima VTEC for Parit Raja (PR) ranged from 38-100 TECU, which is generally higher than those of Sipitang, which ranged from 3042 TECU and corrected amplitude scintillation index $S_{4}$ no greater than 0.1 and average on the order of 0.03-0.04 [97].

The dual frequency corrected scintillation index $S_{4}$ experimental results are in complete agreement with the 4parameter ionospheric model presented in this journal paper.

\section{Conclusions}

In conclusion we have demonstrated in theory and simulations that the 1-parameter ionospheric channel model that is based on estimating the ionospheric delay is insufficient to enable the GNSS receiver to reliably acquire and track GNSS signals under severe ionospheric conditions.

In order to solve this problem Giftet Inc. has proposed a 4parameter ionospheric model. The 4-parameter ionospheric model is based on estimating four parameters: ionospheric delay, Doppler, amplitude, and phase scintillation. The 4parameter ionospheric model presents a revolutionary advancement of our understanding of the ionospheric effects in the RF signals.

In order to accurately estimate the ionospheric Doppler, amplitude, and phase scintillations we have performed some of the most amazing mathematical calculations.

This paper presents one of the most daunting tasks ever undertaken by a single scholar to accurately model, analyze, and simulate very accurately the entire description of the ionospheric channel effects.

After the theory and simulation presented in this paper are completely verified and accurately tested, the implementation thereof in a single frequency GNSS receiver will enable it to perform just as good as or even better than a differential GNSS receiver.

\section{Acknowledgement}

This work was supported by Giftet Inc. executive office.

I want to profoundly thank the MathWorks at Natick, Massachusetts for providing a sponsored MATLAB licence [98] to Giftet Inc. as part of the Indoor Geolocation Systems MATLAB Library development that will enable the results of this work to be published in Dr. Progri pioneer publication Indoor Geolocation Systems-Theory and Applications. Vol. I (Not yet available in print) [99].

This journal paper is dedicated to four special men in my life: my grandfather, Xhevdet Progri, my dear father, Fiqiri Progri, my father's first cousin Dr. Peter Demir, and the forty-first President of the United States of America, George H.W. Bush. 


\section{References}

[1] Anon., "Atmosphere of Earth," Wikipedia, the free encyclopedia,

Dec.

2019,

http://en.wikipedia.org/wiki/Earth's atmosphere.

[2] Anon., "Ionosphere," Wikipedia, the free encyclopedia, Nov. 2019, http://en.wikipedia.org/wiki/Ionosphere.

[3] Anon., "Water vapour," Wikipedia, the free encyclopedia, Feb. 2019, http://en.wikipedia.org/wiki/Water_vapour or http://en.wikipedia.org/wiki/Water_vapor.

[4] Anon., "Atmospheric ducting," Wikipedia, the free encyclopedia,

Nov.

2019 ,

http://en.wikipedia.org/wiki/Atmospheric_ducting.

[5] Anon., "Refractive index," Wikipedia, the free encyclopedia,

Nov.

2019 ,

https://en.wikipedia.org/wiki/Refractive_index.

[6] Anon., "Ionospheric reflection," Wikipedia, the free encyclopedia,

Nov.

2019 ,

http://en.wikipedia.org/wiki/Ionospheric_reflection.

[7] Anon., "Refraction," Wikipedia, the free encyclopedia, Nov. 2019, http://en.wikipedia.org/wiki/Refraction.

[8] Anon., "Constant," Wikipedia, the free encyclopedia, Jan. 2019, http://en.wikipedia.org/wiki/Constant.

[9] Anon., "Ionospheric absorption," Wikipedia, the free encyclopedia,

Nov.

2019, http://en.wikipedia.org/wiki/Ionospheric_absorption.

[10] Anon., "Attenuation," Wikipedia, the free encyclopedia, Nov. 2019, http://en.wikipedia.org/wiki/Attenuation.

[11] Anon., "Ionospheric delay," Navipedia, the GNSS encyclopedia,

Mar.

2019 , http://navipedia.net/index.php/Ionospheric_Delay.

[12] Anon., "Ionospheric delay," Althos, Wireless dictionary, 2019, http://wirelessdictionary.com/Wireless-DictionaryIonospheric-Delay-Definition.html.

[13] J.A. Klobuchar, "Ionospheric effects on GPS," GPS World Mag., vol. 2, no. 4, Apr. 1991.

[14] C. Ouzeau, C. Macabiau, B. Roturier, M. Mabilleau, L. Azoulai, J. Levan, F. Besse, "Ionospheric delay estimation in a single frequency mode for civil aviation," in Proc. $21^{\text {st }}$ Inter. Tech. Mtg. Sat. Div. ION (ION GNSS 2008), Savannah, GA, pp. 1148-1158, Sep. 2008

[15] G. Marucco, F. Dominici, G. Falco, F. Dovis, “A study of DGPS performance in case of limited bandwidth availability," in Proc. $22^{\text {nd }}$ Inter. Tech. Mtg. Sat. Div. ION (ION GNSS 2009), Savannah, GA, pp. 1195-1202, Sep. 2009.
[16] Anon., "RTCM recommended standards for differential GNSS service,” RTCM SC-104, ver. 2.3, Aug., 2001.

[17]Anon., "Dusk," Wikipedia, the free encyclopedia, Nov. 2019, http://en.wikipedia.org/wiki/Dusk.

[18] Anon., "Dawn," Wikipedia, the free encyclopedia, Nov. 2019, http://en.wikipedia.org/wiki/Dawn.

[19]I.F. Progri, "Performance evaluation of the direct acquisition of GPS-like VBOC signals against noise and interference-technical report," J. Geol. Geoinfo. Geointel., vol. 2018, article ID 2018071603, 18 pg., Nov. 2018. DOI: http://doi.org/10.18610/JG3.2018.071603, http:/giftet.com/JG3/2018/071603.pdf.

[20]Anon., "Free-space path loss," Wikipedia, the free encyclopedia, Feb. 2019, http://en.wikipedia.org/wiki/Free-space_path_loss.

[21]Anon., "Inverse square law," Wikipedia, the free encyclopedia, $\quad$ Feb. 2019 http://en.wikipedia.org/wiki/Inverse-square_law or http://en.wikipedia.org/wiki/Inverse_square_law.

[22] Anon., "Watt," Wikipedia, the free encyclopedia, Oct. 2019, http://en.wikipedia.org/wiki/Watt.

[23] Anon., "Speed of light," Wikipedia, the free encyclopedia, Feb. 2019, http://en.wikipedia.org/wiki/Speed of light.

[24] Anon., "Coulomb," Wikipedia, the free encyclopedia, Oct. 2012, http://en.wikipedia.org/wiki/Coulomb.

[25]N. Matteo, Y. Morton, P. Chandrasekaran, F. van Graas, "Higher order ionosphere errors at Arecibo, Millstone, and Jicamarca," in Proc. $22^{\text {nd }}$ Inter. Tech. Mtg. Sat. Div. ION (ION GNSS 2009), Savannah, GA, pp. 2739-2740, Sep. 2009.

[26]Anon., "Relative permittivity," Wikipedia, the free encyclopedia, Dec. 2019 http://en.wikipedia.org/wiki/Relative_permittivity.

[27] Anon., "Permeability (electromagnetism)," Wikipedia, the free encyclopedia, 2019, http://en.wikipedia.org/wiki/Permeability_(electromagnet ism).

[28]F. Meyer, R. Bamler, N. Jakowski, T. Fritz, “The potential of low-frequency SAR systems for mapping ionospheric TEC distributions," IEEE Geosci. Rem. Sens. Letters, vol. 3, no. 4, pp. 560-564, 2006, DOI: https://doi.org/10.1109/LGRS.2006.882148.

[29]I.F. Progri, "The study of the refractive index in anisotropic absorptive turbulent magnetized plasma," J. Geol. Geoinfo. Geointel., vol. 2019, article ID 
2019071602, 22 pg., Nov. 2019. DOI: http://doi.org/10.18610/JG3.2019.071602,

http://giftet.com/JG3/2019/071602.pdf.

[30] Anon., "Electron," Wikipedia, the free encyclopedia, Sep. 2019, http://en.wikipedia.org/wiki/Electron.

[31]Anon., "Vacuum permittivity," Wikipedia, the free encyclopedia, Sep.

2019 , http://en.wikipedia.org/wiki/Vacuum_permittivity.

[32]Anon., "Plasma_(physics)," Wikipedia, the free encyclopedia, Feb. 2019, http://en.wikipedia.org/wiki/Plasma_(physics).

[33]Anon., "Plasma_(oscillation)," Wikipedia, the free encyclopedia,

Oct. 2019 , http://en.wikipedia.org/wiki/Plasma_oscillation.

[34] R. Di Corato, S. Falzini, A. Beccarini, A. Caporali, C. Morini, T. Pepi, "SISE prediction and iono/tropo corrections in a local element augmentation system," in Proc. 2007 Nat. Tech. Mtg. ION, San Diego, CA, pp. 979989, Jan. 2007.

[35] Anon., "Classical electron radius," Wikipedia, the free encyclopedia,

Mar.

2019 , http://en.wikipedia.org/wiki/Classical_electron_radius.

[36] Anon., "Total electron content," Wikipedia, the free encyclopedia,

Apr.

2019, http://en.wikipedia.org/wiki/Total_electron_content.

[37] Anon., "Physical constants,"

2019 , http://ecse.rpi.edu/ schubert/Light-Emitting-Diodes-dotorg/Physicalconstants.pdf.

[38] P.M. Kintner, Jr., “A beginner's guide to space weather and GPS," Lecture Notes, 11 pg., Feb., 2008, http://gps.ece.cornell.edu/SpaceWeatherIntro_ed2_10-3106_ed.pdf.

[39] P.M. Kintner, B.M. Ledvina, E.R. de Paula, "GPS and ionospheric scintillations," Space Weather, vol. 5, no. S09003, pp. 1-23, 2007, DOI: http://doi.org/10.1029/2006SW000260.

[40] S. Zhang, S. Lim, C. Rizos, J. Guo, "Atmosphere decomposition for VRS based network-RTK system," in Proc. $22^{\text {nd }}$ Inter. Tech. Mtg. Sat. Div. ION (ION GNSS 2009), Savannah, GA, pp. 2707-2716, Sep. 2009.

[41] C-O. Andrei, R. Chen, H. Kuusniemi, M. HernandezPajares, J.M. Juan, D. Salazar, "Ionosphere effect mitigation for single-frequency precise point positioning," in Proc. $22^{\text {nd }}$ Inter. Tech. Mtg. Sat. Div. ION (ION GNSS 2009), Savannah, GA, pp. 2508-2517, Sep. 2009.
[42] S. Sunda, S.M. Regar, S. Shrivastava, R. Acharya, P.V. Khekale, M.R. Sivaraman, "TEC estimation and its validation for real time implementation of satellite navigation in GAGAN," in Proc. 2007 Nat. Tech. Mtg. ION, San Diego, CA, pp. 1050-1055, Jan. 2007.

[43]F. Ghafoori, "Correcting the negative values of the retrieved ionospheric electron density profiles using the NNLS algorithm," in Proc. 22 $2^{\text {nd }}$ Inter. Tech. Mtg. Sat. Div. ION (ION GNSS 2009), Savannah, GA, pp. 2518-2529, Sep. 2009.

[44] S.K. Mitra, "Report on the present state of our knowledge of the ionosphere," University of Calcutta, pp. 131-215, 1935.

[45] R.B. Langley, "Propagation of the GPS signals," GPS for Geodesy, $2^{\text {nd }}$ ed. book chapter in Lecture Notes in Earth Sciences, Berlin: Springer-Verlag, vol. 60, pp 103-140, 1996.

[46] S. Datta-Barua, P.H. Doherty, S.H. Delay, T. Dehel, J.A. Klobuchar, "Ionospheric scintillation effects on single and dual frequency GPS positioning," in Proc. $16^{\text {th }}$ Inter. Tech. Mtg. Sat. Div. ION (ION GPS/GNSS 2003), Portland, OR, pp. 336-346, Sep. 2003.

[47]Anon., "Gradient-index optics.," Wikipedia, the free encyclopedia, Nov. 2019, https://en.wikipedia.org/wiki/Gradient-index_optics.

[48]Anon., "Tensor," Wikipedia, the free encyclopedia, Nov. 2019, https://en.wikipedia.org/wiki/Tensor.

[49] G.B. Arfken, H.J., Weber, Mathematical Methods for Physicists, San Diego, CA: Academic Press, 1995.

[50] S. Maus, "Conductivity of the ionosphere," CIRES, University of Colorado, 15 pg., Jan. 2006, http://geomag.us/info/Geomag_tutorials/Maus_ionospheri c_conductivity.pdf.

[51]J. Blanch, "An ionospheric estimation algorithm for WAAS based on Kriging," in Proc. $15^{\text {th }}$ Inter. Tech. Mtg. Sat. Div. ION (ION GPS 2002), Portland, OR, pp. 816-823, 2002.

[52] J. Blanch, "Using Kriging to bound satellite ranging errors due to the ionosphere," Ph.D. Dissertation, Stanford University, Standord, CA, 148 pg., Dec. 2003.

[53]J. Blanch, T. Walter, P. Enge, "A new ionospheric estimation algorithm for SBAS combining kriging and tomography," in Proc. 2004 Nat. Tech. Mtg. ION, San Diego, CA, pp. 524-529, Jan. 2004. 
[54]D. Bilitza, "Ionospheric models for radio propagation studies," in The Review Radio Sci. 1999-2002, edited by W.R. Stone, pp. 625-679, IEEE Press, Piscataway, N. J., 2002.

[55] A.B.O. Jensen, O. Ovstedal, G. Grinde, "Development of a regional ionosphere model for Norway," in Proc. 2008 Nat. Tech. Mtg. ION, San Diego, CA, pp. 893-902, Jan. 2008.

[56]C. Ouzeau, F. Bastide, C. Macabiau, B. Roturier, "Ionospheric code delay estimation in a single frequency case for civil aviation," in Proc. 19th Inter. Tech. Mtg. Sat. Div. ION (ION GNSS 2006), Fort Worth, TX, pp. 30593069, Sep. 2006.

[57]L. Sparks, A. Komjathy, A.J. Mannucci, , "Estimating SBAS ionospheric delays without grids: the conical domain approach," in Proc. 2004 Nat. Tech. Mtg. ION, San Diego, CA, pp. 530-541, Jan. 2004.

[58] C. Gernot, S.K. Shanmugam, K. O’Keefe, G. Lachapelle, "A novel L1 and L2C combined detection scheme for enhanced GPS acquisition," in Proc. $20^{\text {th }}$ Inter. Tech. Mtg. Sat. Div. ION (ION GNSS 2007), Fort Worth, TX, pp. 219230, Sep. 2007.

[59] T. Sakai, K. Matsunaga, K. Hoshinoo, K. Ito, T. Walter, "Mitigating ionospheric threat using a dense monitoring network," in Proc. 20th Inter. Tech. Mtg. Sat. Div. ION (ION GNSS 2007), Fort Worth, TX, pp. 927-938, Sep. 2007.

[60] S. Rajagopal, T. Walter, S. Datta-Barua, J. Blanch, T. Sakai, "Correlation structure of the equatorial ionosphere," in Proc. 2004 Nat. Tech. Mtg. ION, San Diego, CA, pp. 542-550, Jan. 2004.

[61] Anon., "Earth radius," Wikipedia, the free encyclopedia, Sep. 2019, http://en.wikipedia.org/wiki/Earth_radius.

[62] R.F. Leandro, M.C. Santos, R.B. Langley, "PPP-based ionospheric activity monitoring," in Proc. $20^{\text {th }}$ Inter. Tech. Mtg. Sat. Div. ION (ION GNSS 2007), Fort Worth, TX, pp. 2849-2853, Sep. 2007.

[63] J. Jakobsen, P. Knudsen, A.B.O. Jensen, "Principle component analysis on absolute TEC values and its impact on Kalman filtering when using a priori information," in Proc. 2008 Nat. Tech. Mtg. ION, San Diego, CA, pp. 884892, Jan. 2008.

[64] K. Chen, Y. Gao, "Ionospheric effect mitigation for realtime single-frequency precise point positioning,"
Navigation, vol. 55, no. 3, pp. 205-213, fall 2008, DOI: https://doi.org/10.1002/j.2161-4296.2008.tb00430.x.

[65] S.E. Langel, S.M. Khanafseh, F-C. Chan, B. Pervan, "Tightly coupled GPS/INS integration for differential carrier phase navigation systems using decentralized estimation," in Proc. IEEE/ION PLANS 2010, Indian Wells, CA, pp. 397-409, May 2010, DOI: https://doi.org/10.1109/PLANS.2010.5507177.

[66] M. Joerger, J. Neale, B. Pervan, S. Datta-Barua, "Measurement error models and fault-detection algorithms for multi-constellation navigation systems," in Proc. IEEE/ION PLANS 2010, Indian Wells, CA, pp. 927-946, May 2010, DOI: https://doi.org/10.1109/PLANS.2010.5507228.

[67]K. Nishikawa, S. Fujita, Y. Kubo, S. Sugimoto, "PPP based on GR models with estimating tropospheric and ionospheric delays," in Proc. $22^{\text {nd }}$ Inter. Tech. Mtg. Sat. Div. ION (ION GNSS 2009), Savannah, GA, pp. 24962507, Sep. 2009.

[68] T. Yanase, H. Tanaka, M. Ohashi, Y. Kubo, S. Sugimoto, "Long baseline relative positioning with estimating ionosphere and troposphere gradients," in Proc. $23^{\text {rd }}$ Inter. Tech. Mtg. Sat. Div. ION (ION GNSS 2010), Portland, OR, pp. 196-206, Sep. 2010

[69]B. Pervan, F.C. Chan, "System concepts for cycle ambiguity resolution and verification for aircraft carrier landings," in Proc. $14^{\text {th }}$ Inter. Tech. Mtg. Sat. Div. ION (ION GPS 2001), Salt Lake City, UT, pp. 1228-1237, Sep. 2001.

[70] G. McGraw, T. Murphy, M. Brenner, S. Pullen, A.J. Van Dierendonck, "Development of the LAAS accuracy models," in Proc. 13th Inter. Tech. Mtg. Sat. Div. ION (ION GPS 2000), Salt Lake City, UT, pp. 1212-1223, Sep. 2000.

[71]R. Niles, C.A. Shively, "Development of an error model for locally-achieved performance of WAAS," in Proc. 2006 Nat. Tech. Mtg. ION, Monterey, CA, pp. 342-357, Jan. 2006.

[72] C.A. Shively, "Ranging source fault integrity concepts for a local airport monitor for WAAS," in Proc. 2006 Nat. Tech. Mtg. ION, Monterey, CA, pp. 413-431, Jan. 2006.

[73] J. Seo, J. Rife, S. Pullen, T. Walter, P. Enge, "Field data analysis for a range-based local airport monitor for WAAS," in Proc. 2006 Nat. Tech. Mtg. ION, Monterey, CA, pp. 748-758, Jan. 2006. 
[74] B. Bonet, I. Alcantarilla, D. Flament, C. Rodriguez, N. Zarraoa, "The benefits of multi-constellation GNSS: reaching up even to single constellation GNSS users," in Proc. 22 $2^{\text {nd }}$ Inter. Tech. Mtg. Sat. Div. ION (ION GNSS 2009), Savannah, GA, pp. 1268-1280, Sep. 2009.

[75] Neri, P., Macabiau, C., Azoulai, L., Muller, J., “GBAS NSE model for CAT II/III autoland simulations," in Proc. IEEE/ION PLANS 2010, Indian Wells, CA, pp. 694-707, May 2010 ,

DOI:

https://doi.org/10.1109/PLANS.2010.5507222.

[76] D. Wang, K. O'Keefe, “Benefit of partial L2C availability to estimate ionospheric delay for dual-frequency GPS ambiguity resolution," in Proc. IEEE/ION PLANS 2010, Indian Wells, CA, pp. 44-52, May 2010, DOI: https://doi.org/10.1109/PLANS.2010.5507199.

[77] L.J. Nickisch, M.A. Hausman, S.V. Fridman, "Range rate - Doppler correlation for HF propagation in traveling ionospheric disturbance environments," Radio Sci., vol. 41, RS5S39, Oct. 2006, DOI: http://doi.org/10.1029/2005RS003358.

[78] C. Zhou, Z. Zhao, G. Yang, G. Chen, Y. Hu, Y. Zhang, "Evidence of low-latitude daytime large-scale traveling ionospheric disturbances observed by high-frequency multistatic backscatter sounding system during a geomagnetically quiet period," J. Geophys. Res., vol. 117, A06302, pp. 1-8, 2012, DOI: http://doi.org/10.1029/2012JA017605.

[79] J. Chum, R. Athieno, J. Baše, D. Burešová, F. Hruška, J. Laštovicka, L.A. McKinnell, T. Šindelárová, "Statistical investigation of horizontal propagation of gravity waves in the ionosphere over Europe and South Africa," J. Geophys. Res., vol. 117, A03312, pp. 1-13, 2012, DOI: http://doi.org/10.1029/2011JA017161.

[80] G.V. Jandieri, A. Ishimaru, K. Yasumoto, A.G. Khantadze, V.G. Jandieri, “Angle-of-arrival of radio waves scattered by turbulent collisional magnetized plasma layer," Inter. J. Micro. Opti. Techn., vol. 4, no 3, pp. 160-169, 2009, http://ijmot.com/ijmot/uploaded/Mq22242009206096Kw. pdf.

[81] J.R. Wright, "GPS carrier phase processing," in Proc. 18 Inter. Tech. Mtg. Sat. Div. ION (ION GNSS 2005), Long Beach, CA, pp. 584-591, Sep. 2005.

[82] C., Gernot, K., O’Keefe, G., Lachapelle, “Combined L1 / L2C tracking scheme for weak signal environments," in
Proc. $21^{\text {st }}$ Inter. Tech. Mtg. Sat. Div. ION (ION GNSS 2008), Savannah, GA, pp. 1758-1772, Sep. 2008.

[83]Anon., "Conversion of units," Wikipedia, the free encyclopedia, Nov. 2019, http://en.wikipedia.org/wiki/Conversion_of_units.

[84]B. Ventre, J.L. Garrison, M.H. Boehme, J.S. Haase, "Implementation and testing of open-loop tracking for airborne GPS occultation measurements," in Proc. 19th Inter. Tech. Mtg. Sat. Div. ION (ION GNSS 2006), Fort Worth, TX, pp. 620-633, Sep. 2006.

[85]T.L. Beach, T.R. Pedersen, M.J. Starks, S.-Y. Su, "Estimating the amplitude scintillation index from sparsely sampled phase screen data," Radio Sci., vol. 39, no. RS5001, pp. 1-17, Sep. 2004, DOI: http://doi.org/10.1029/2002RS002792, http://dtic.mil/dtic/tr/fulltext/u2/a429128.pdf.

[86] Anon., "Intensity," Wikipedia, the free encyclopedia, Dec. 2019, http://en.wikipedia.org/wiki/Intensity_(physics).

[87] J. Aarons, "The longitudinal morphology of equatorial Flayer irregularities relative to their occurrence," Space Sci. Rev., vol. 63, pp 209-243, 1993, http://link.springer.com/article/10.1007\%2FBF00750769.

[88] K.M. Groves, S. Basu, E.J. Weber, M. Smitham, H. Kuenzler, C.E. Valladares, R. Sheehan, E. MacKenzie, J.A. Secan, P. Ning, W.J. McNeill, D.W. Moonan, M.J. Kendra, "Equatorial scintillation and systems support," Radio Sci., vol. 32, no. 5, pp. 2047-2064, 1997, DOI: http://doi.org/10.1029/97RS00836.

[89]R.M. Thomas, M.A. Cervera, K. Eftaxiadis, S.L. Manurung, S.S. Effendy, A.G. Ramli, W.S. Hassan, H. Rahman, M.N. Dalimin, K.M. Groves, Y. Wang, “A regional GPS receiver network for monitoring equatorial scintillation and total electron content," Radio Sci, vol. 36, no. 6, pp. 1545-1557, Nov.-Dec. 2001, DOI: http://doi.org/10.1029/2000RS00252.

[90]E.J. Fremouw, R.C. Livingston, D.A. Miller, "On the statistics of scintillating signals," J. Atmos. Terr. Phys., vol. 42, no. 8, pp. 717-731, Aug. 1980, DOI: https://doi.org/10.1016/0021-9169(80)90055-0.

[91]K.C. Yeh, C.H. Liu, "Radio wave scintillations in the ionosphere," in Proc. IEEE, vol. 70, no. 4, pp. 324-360, Apr. 1982, DOI: https://doi.org/10.1109/PROC.1982.12313.

[92] G.V. Jandieri, A. Ishimaru, V.G. Jandieri, A.G. Khantadze, Z.M. Diasamidze, "Model computations of angular power 
spectra for anisotropic absorptive turbulent magnetized plasma," Progr. Electromagn. Res., PIER 70, pp. 307-328, 2007 ,

http://jpier.org/PIER/pier70/16.07013103.Jandieri.IJKD.p df.

[93] G.V. Jandieri, A. Ishimaru, V.G. Jandieri, A.G. Khantadze, I.B. Shirokov, Yu.B. Gimpilevich, Zh.M. Diasamidze, "Wave structure functions of multiply scattered electromagnetic waves by anisotropic magnetized turbulent plasma layer," PIERS Online, vol. 4, no. 7, pp. 785-790,

2008 , http://piers.org/piersonline/pdf/Vol4No7Page785to790.pd f.

[94] G.V. Jandieri, A. Ishimaru, V.G. Jandieri, A.G. Khantadze, N.Kh. Gomidze, K.V. Kotetishvili, T.N. Bzhalava, Sh.V. Dekanosidze, I.S. Surmanidze, "Peculiarities of the spatial spectrum of scattered electromagnetic waves by anisotropic collisional magnetized turbulent plasma layer," in Symp. Progr. Electromag. Res., Beijing, China, pp. 8590, Mar. 2009.

i This paper presents one of the most daunting tasks ever undertaken by a single scholar to very accurately model, analyze, and simulate the entire description of the ionospheric channel effects.

ii These four parameters are not really independent of each other but they start our as independent parameters and they can be observed and measured independently of each other; hence, a more appropriate definition would be semi-independent.

iii Ditto i.

iv It is assumed that most of the conductivity comes as result of the presence of a magnetic field ignoring the average time interval between collisions as a result of the drift movements of the electrons and the effects from free ions [50]. $\checkmark$ el(s) means electon(s)

${ }^{v i}$ It is possible to remove only the first order ionospheric refraction terms (up to the $99 \%$ of the total refractive effect) using their dependence on frequency [34].

vii Equation (9) in Matteo et al. 2009, [24] is an equivalent expression for $a=$ $0.5 C_{x}$ given by (41); however, there is an error in units in (9) in Matteo et al. 2009, [24].

viii Equation (41) uses the same units; i.e., $\frac{\mathrm{els}^{-1} \mathrm{CVm}}{\mathrm{kg}}=\frac{\mathrm{els}^{-1} \mathrm{Wms}}{\mathrm{kg}}=\frac{\mathrm{els}^{-1} \mathrm{~m}^{2} \mathrm{~ms}}{\mathrm{~s}^{3}}=$ els ${ }^{-1} \mathrm{~m}^{3} \mathrm{~s}^{-2}$ [22], [24].

ix The earlies calculation of the ionospheric critical frequency comes from (Mitra 1935, pg. 153 [44]). Mitra gives a value of $5.4 \times 10^{6} \mathrm{~Hz} \equiv 5.4 \mathrm{MHz}$ for the ionospheric critical frequency in Calcutta (presently Kolkata, India).

x The ionospheric delay, $D_{i, t}^{S}(t)$, is a subcomponent of the $D_{e}^{S}(t)$ or assuming that all other channel effects are ignored then
[95] C.S. Carrano, K.M. Groves, “Temporal decorrelation of GPS satellite signals due to multiple scattering from ionospheric irregularities," in Proc. 23 ${ }^{\text {rd }}$ Inter. Tech. Mtg. Sat. Div. ION (ION GNSS 2010), Portland, OR, pp. 361374, Sep. 2010.

[96]L. Zhang, Y.T. Morton, “Tracking GPS signals under ionosphere scintillation conditions," in Proc. $22^{\text {nd }}$ Inter. Tech. Mtg. Sat. Div. ION (ION GNSS 2009), Savannah, GA, pp. 227-234, Sep. 2009.

[97] M. Abdullah, A.F.M. Zain, Y.H. Ho, S. Abdullah, “TEC and scintillation study of equatorial ionosphere: a month campaign over Sipitang and Parit Raja stations, Malaysia," Amer. J. Eng. \& Appl. Sci., vol. 2, no. 1, pp. 44-49, 2009.

[98]Anon, "MATLAB 2018b," The MathWorks, Inc., Natick, MA, Copyright (C) 1994-2018, The MathWorks, Inc., http://mathworks.com/products/new_products/release200 6b.html?requestedDomain=mathworks.com.

[99]I.F. Progri, Indoor Geolocation Systems-Theory and Applications. Vol. I, $1^{\text {st }}$ ed., Worcester, MA: Giftet Inc., $\sim 800$ pp., $\sim 2020$ (not yet available in print).

$D_{e}^{S}(t)=D_{i, t}^{s}(t) ; t \in[0, T)$

xi The detailed discussion of the Snell's law, Fresnel's equations, and ray's propagation path that provide a complete derivation and interpretation of the ray path delay will be published in a separate journal paper.

xii Refractivity of ionosphere is actually a rank-2 (or second) tensor (a $3 \times 3$ matrix) or a mathematical object analogous to but more general than a vector, represented by an array of components that are functions of the coordinates of a space [5]. Tensors are geometric objects that describe linear relations between geometric vectors, scalars, and other tensors [48]. In this case the propagation of the RF signal cannot simply be described by refractive indices except for polarizations along principal axes [5]

xiii Andrei et al. (2009, [41]) for $h_{i}$ a value in the range of $300-500 \mathrm{~km}$ is typical.

xiv Blanch et. al. (2004, [53]) provide a model to include two properties of the ionosphere: the vertical density profile (ignored in the thin shell models which will be subject of a separate journal paper) and the decorrelation of the ionospheric delay as a function of distance (ignored in the tomographic models.) To this purpose, we generalize the two dimensional model that is used in Kriging [51]-[53], [55] and is based on multivariate Gaussian random fields [51], [52], [60] by introducing $p$ layers instead of a single thin shell. Another approach is "the conical domain approach" proposed by Sparks et al. (2004, [57]).

xv There was a typo in (2) in [69] the '+' should be '-' as in (96) or (89).

${ }^{x v i}$ The detailed discussion and the complete derivations and interpretation of this section will be published in a separate journal paper. 